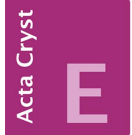
COMMUNICATIONS

ISSN 2056-9890

\section{Crystal structure of the inclusion} complex 25-benzoylmethoxy-5,11,17,23tetra-tert-butyl-26,27,28-trihydroxy$2,8,14,20$-tetrathiacalix [4]arene-tetraethylammonium chloride (1/1)

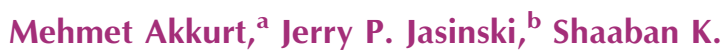
Mohamed, ${ }^{c, d}$ Omran A. Omran ${ }^{e, f}$ and Mustafa R. Albayati $^{\mathrm{g} *}$

${ }^{a}$ Department of Physics, Faculty of Sciences, Erciyes University, 38039 Kayseri, Turkey, 'bepartment of Chemistry, Keene State College, 229 Main Street, Keene, NH 03435-2001, USA, ${ }^{\mathbf{c}}$ Chemistry and Environmental Division, Manchester Metropolitan University, Manchester M1 5GD, England, 'Chemistry Department, Faculty of Science, Minia University, 61519 El-Minia, Egypt, ${ }^{\mathbf{e}}$ Medical Laboratory Department, College of Science, Majmaah University, 11932, Saudi Arabia, ${ }^{\mathfrak{f}}$ Chemistry Department, Faculty of Science, Sohag University, 82524 Sohag, Egypt,

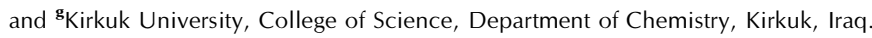
*Correspondence e-mail: shaabankamel@yahoo.com

Received 8 September 2015; accepted 5 October 2015

Edited by D.-J. Xu, Zhejiang University (Yuquan Campus), China

The asymmetric unit of the title compound, $\mathrm{C}_{48} \mathrm{H}_{54} \mathrm{O}_{5} \mathrm{~S}_{4} \cdot \mathrm{N}\left(\mathrm{C}_{2} \mathrm{H}_{5}\right)_{4}{ }^{+} \cdot \mathrm{Cl}^{-}$, contains two tetra-tert-butyl[(benzoyl)methoxy]-trihydroxy-tetrathiacalix[4]arene molecules, two tetraethylammonium cations and two chloride anions. The two calixarene molecules in the asymmetric unit each display a cone conformation. There are no significant differences between the two independent molecules. The guest species do not sit within the calixarene 'buckets'. In the crystal, extensive $\mathrm{O}-\mathrm{H} \cdots \mathrm{O}, \mathrm{O}-\mathrm{H} \cdots \mathrm{S}$ and $\mathrm{O}-\mathrm{H} \cdots \mathrm{Cl}$ hydrogen bonds and weak $\mathrm{C}-\mathrm{H} \cdots \mathrm{O}, \mathrm{C}-\mathrm{H} \cdots \mathrm{S}$ and $\mathrm{C}-$ $\mathrm{H}$.. Cl interactions link the thiacalixarene molecules, tetraethylammonium cations and chloride anions, forming a threedimensional network encompassing channels running parallel to the $a$-axis direction. The structure contains a solventaccessible void of 76 (3) $\AA^{3}$, but no solvent molecule could reasonably be located. The crystal studied was an inversion twin with a $0.57(8): 0.43(8)$ domain ratio.

Keywords: crystal structure; co-crystal; p-tert-butylthiacalix[4]arene; phase transfer catalysis; tetraethylammonium chloride; alkylation; hydrogen bonding.

CCDC reference: 1429489

\section{Related literature}

For chemistry background and applications of thiacalixarenes, see: Gutsche (1998); Shokova \& Kovalev (2003); Stoikov et al. (2003); Agrawal \& Pancholi (2007); Ben Ali et al. (2001); Desroches et al. (2003); Higuchi et al. (2000); Iki \& Miyano (2001); Morohashi et al. (2002); Odo et al. (2000, 2001); Omran \& Antipin (2014). Many calixarene derivatives are being used in both environmental and biomedical monitoring, see: McMahon et al. (2003).

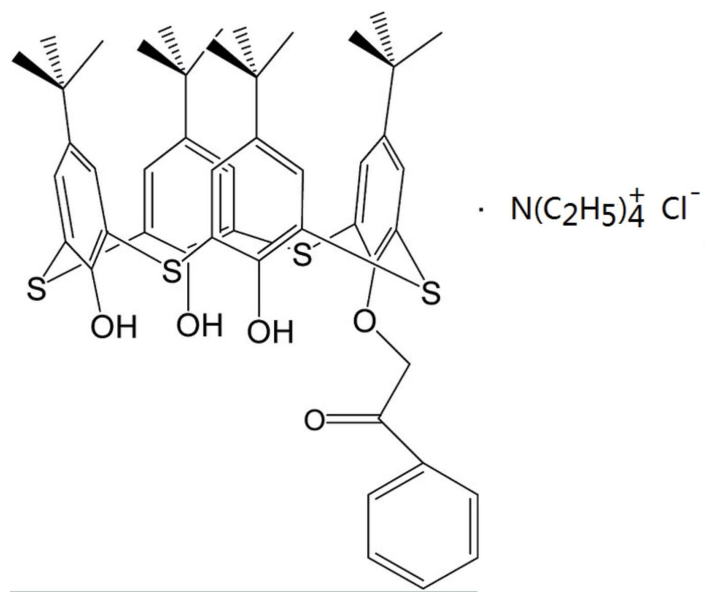

\section{Experimental}

2.1. Crystal data

$\mathrm{C}_{48} \mathrm{H}_{54} \mathrm{O}_{5} \mathrm{~S}_{4} \cdot \mathrm{C}_{8} \mathrm{H}_{20} \mathrm{~N}^{+} \cdot \mathrm{Cl}^{-}$

$M_{r}=1004.85$

Monoclinic, $P 2_{1}$

$a=19.4338(6) \AA$

$b=14.2147$ (3) ̊

$c=20.8631(6) \AA$

$\beta=103.184(3)^{\circ}$

$$
\begin{aligned}
& V=5611.4(3) \AA^{3} \\
& Z=4 \\
& \text { Mo } K \alpha \text { radiation } \\
& \mu=0.26 \mathrm{~mm}^{-1} \\
& T=293 \mathrm{~K} \\
& 0.42 \times 0.38 \times 0.22 \mathrm{~mm}
\end{aligned}
$$

\subsection{Data collection}

Agilent Xcalibur, Eos, Gemini diffractometer

Absorption correction: multi-scan (CrysAlis PRO; Agilent, 2014)

$T_{\min }=0.922, T_{\max }=1.000$

40706 measured reflections 25992 independent reflections 18033 reflections with $I>2 \sigma(I)$ $R_{\text {int }}=0.035$

\subsection{Refinement}

$R\left[F^{2}>2 \sigma\left(F^{2}\right)\right]=0.079$

$w R\left(F^{2}\right)=0.241$

$S=1.05$

25992 reflections

1208 parameters

8 restraints
$\mathrm{H}$-atom parameters constrained $\Delta \rho_{\max }=0.98 \mathrm{e} \AA^{-3}$ $\Delta \rho_{\min }=-0.88{\mathrm{e} \AA^{-3}}^{-3}$

Absolute structure: Flack (1983) Absolute structure parameter: $0.43(8)$ 
Table 1

Hydrogen-bond geometry $\left(\AA,^{\circ}\right)$.

\begin{tabular}{|c|c|c|c|c|}
\hline$D-\mathrm{H} \cdots A$ & $D-\mathrm{H}$ & $\mathrm{H} \cdots A$ & $D \cdots A$ & $D-\mathrm{H} \cdots A$ \\
\hline $\mathrm{O} 1-\mathrm{H} 1 O \cdots \mathrm{S} 2$ & 0.82 & 2.61 & $3.110(4)$ & 121 \\
\hline $\mathrm{O} 1-\mathrm{H} 1 O \cdots \mathrm{O} 2$ & 0.82 & 1.85 & $2.581(6)$ & 147 \\
\hline $\mathrm{O} 2-\mathrm{H} 2 \mathrm{O} \cdots \mathrm{Cl} 2$ & 0.82 & 2.30 & $3.048(10)$ & 152 \\
\hline $\mathrm{O} 3-\mathrm{H} 3 O \cdots \mathrm{S} 3$ & 0.82 & 2.57 & $3.077(4)$ & 121 \\
\hline $\mathrm{O} 3-\mathrm{H} 3 O \cdots \mathrm{O} 2$ & 0.82 & 1.91 & $2.567(6)$ & 136 \\
\hline $\mathrm{O} 1^{\prime}-\mathrm{H} 6 O \cdots \mathrm{S} 2^{\prime}$ & 0.82 & 2.60 & $3.105(4)$ & 121 \\
\hline $\mathrm{O} 1^{\prime}-\mathrm{H} 6 O \cdots \mathrm{O} 2^{\prime}$ & 0.82 & 1.85 & $2.580(5)$ & 147 \\
\hline $\mathrm{O} 1^{\prime}-\mathrm{H} 6 O \cdots \mathrm{Cl} 1^{\mathrm{i}}$ & 0.82 & 2.81 & $3.338(11)$ & 124 \\
\hline $\mathrm{O} 2^{\prime}-\mathrm{H} 7 O \cdots \mathrm{Cl}^{\mathrm{i}}$ & 0.82 & 2.27 & $3.006(10)$ & 150 \\
\hline $\mathrm{O}^{\prime}-\mathrm{H} 8 O \cdots \mathrm{S}^{\prime}$ & 0.82 & 2.57 & $3.078(4)$ & 121 \\
\hline $\mathrm{O}^{\prime}-\mathrm{H} 8 \mathrm{O} \cdots \mathrm{O} 2^{\prime}$ & 0.82 & 1.90 & $2.567(5)$ & 138 \\
\hline $\mathrm{C} 103-\mathrm{H} 10 L \cdots \mathrm{Cl} 2$ & 0.97 & 2.43 & $3.397(17)$ & 174 \\
\hline $\mathrm{C} 106-\mathrm{H} 10 V \cdots \mathrm{Cl} 2$ & 0.96 & 2.64 & $3.382(19)$ & 135 \\
\hline $\mathrm{C} 202-\mathrm{H} 20 \mathrm{I} \cdots \mathrm{O} 5$ & 0.96 & 2.58 & $3.459(16)$ & 152 \\
\hline $\mathrm{C} 203-\mathrm{H} 20 L \cdots \mathrm{Cl} 1^{\mathrm{ii}}$ & 0.97 & 2.41 & $3.373(15)$ & 173 \\
\hline $\mathrm{C} 204-\mathrm{H} 20 P \cdots \mathrm{Cl} 2^{\mathrm{ii}}$ & 0.96 & 2.78 & $3.63(2)$ & 147 \\
\hline $\mathrm{C} 206-\mathrm{H} 20 V \cdots \mathrm{Cl} 1^{\mathrm{ii}}$ & 0.96 & 2.55 & $3.353(18)$ & 141 \\
\hline $\mathrm{C} 41-\mathrm{H} 41 A \cdots \mathrm{O} 3$ & 0.97 & 2.45 & $3.318(6)$ & 149 \\
\hline $\mathrm{C} 41-\mathrm{H} 41 B \cdots \mathrm{O} 1$ & 0.97 & 2.46 & $3.253(6)$ & 139 \\
\hline $\mathrm{C} 41^{\prime}-\mathrm{H} 41 C \cdots \mathrm{S} 4^{\prime}$ & 0.97 & 2.87 & $3.414(6)$ & 116 \\
\hline $\mathrm{C} 41^{\prime}-\mathrm{H} 41 C \cdots \mathrm{O}^{\prime}$ & 0.97 & 2.45 & $3.313(6)$ & 148 \\
\hline $\mathrm{C} 41^{\prime}-\mathrm{H} 41 D \cdots \mathrm{O}^{\prime}$ & 0.97 & 2.47 & $3.265(6)$ & 139 \\
\hline $\mathrm{C} 44-\mathrm{H} 44 \cdots \mathrm{O} 2^{\mathrm{iii}}$ & 0.93 & 2.54 & $3.431(7)$ & 160 \\
\hline $\mathrm{C} 44^{\prime}-\mathrm{H} 44^{\prime} \cdots \mathrm{O} 2^{\prime \text { iv }}$ & 0.93 & 2.57 & $3.447(6)$ & 158 \\
\hline $\mathrm{C} 47-\mathrm{H} 47 \ldots \mathrm{O}^{\mathrm{v}}$ & 0.93 & 2.55 & $3.368(6)$ & 146 \\
\hline $\mathrm{C} 47-\mathrm{H} 47 \cdots \mathrm{O}^{\mathrm{v}}$ & 0.93 & 2.45 & $3.255(7)$ & 145 \\
\hline $\mathrm{C} 47^{\prime}-\mathrm{H} 47^{\prime} \cdots \mathrm{O} 4^{\prime \mathrm{vi}}$ & 0.93 & 2.57 & $3.380(6)$ & 146 \\
\hline $\mathrm{C} 47^{\prime}-\mathrm{H}_{4} 47^{\prime} \cdots \mathrm{O}^{\prime \mathrm{vi}}$ & 0.93 & 2.47 & $3.277(7)$ & 145 \\
\hline
\end{tabular}

Symmetry codes: (i) $-x+1, y+\frac{1}{2},-z+1$; (ii) $x, y+1, z$; (iii) $-x+2, y+\frac{1}{2},-z+2$; (iv) $-x+1, y-\frac{1}{2},-z ;(\mathrm{v})-x+2, y-\frac{1}{2},-z+2 ;(\mathrm{vi})-x+1, y+\frac{1}{2},-z$.

Data collection: CrysAlis PRO (Agilent, 2014); cell refinement: CrysAlis PRO; data reduction: CrysAlis PRO; program(s) used to solve structure: SHELXS2014 (Sheldrick, 2008); program(s) used to refine structure: SHELXL2014 (Sheldrick, 2015); molecular graphics: ORTEP-3 for Windows (Farrugia, 2012); software used to prepare material for publication: PLATON (Spek, 2009).

\section{Acknowledgements}

The authors acknowledge support from the Deanship of Scientific Research, and thank Majmaah University for funding this work under project No. 1. JPJ would like to acknowledge the NSF-MRI program (grant No. CHE1039027) for funds to purchase the X-ray diffractometer.

Supporting information for this paper is available from the IUCr electronic archives (Reference: XU5871).

\section{References}

Agilent (2014). CrysAlis PRO. Agilent Technologies Ltd, Yarnton, England. Agrawal, Y. K. \& Pancholi, J. P. (2007). Indian J. Chem. Sect. A, 46, 1377-1382.

Ben Ali, M., Lemiti, M., Jaffrezic-Renault, N., Martelet, C., Chovelon, J. M. \& Ben Ouada, H. (2001). Thin Layer Films, 383, 292-295.

Desroches, C., Lopes, C., Kessler, V. \& Parola, S. (2003). Dalton Trans. pp. 2085-2092

Farrugia, L. J. (2012). J. Appl. Cryst. 45, 849-854.

Flack, H. D. (1983). Acta Cryst. A39, 876-881.

Gutsche, C. D. (1998). Calixarenes Revisited. Monographs in Supramolecular Chemistry, edited by J. F. Stoddart. London: RSC.

Higuchi, Y., Narita, M., Niimi, T., Ogawa, N., Hamada, F., Kumagai, H., Iki, N., Miyano, S. \& Kabuto, C. (2000). Tetrahedron, 56, 4659-4666.

Iki, N. \& Miyano, S. (2001). J. Inclusion Phenom. Macrocyclic Chem. 41, 99105.

McMahon, G., O'Malley, S., Nolan, K. \& Diamond, D. (2003). Arkkivoc, vii, 23-31

Morohashi, N., Iki, N. \& Miyano, S. (2002). J. Syn. Org. Chem. Jpn, 60, 550562.

Odo, J., Inomata, Y., Takeya, H., Miyanari, S. \& Kumagai, H. (2001). Anal. Sci. 17, $1425-1429$.

Odo, J., Kawahara, N., Inomata, Y., Inoue, A., Takeya, H., Miyanari, S. \& Kumagai, H. (2000). Anal. Sci. 16, 963-966.

Omran, A. O. \& Antipin, I. S. (2014). J. Incl Phenom. Macrocycl Chem. 78, 121-126.

Sheldrick, G. M. (2008). Acta Cryst. A64, 112-122.

Sheldrick, G. M. (2015). Acta Cryst. C71, 3-8

Shokova, E. A. \& Kovalev, V. V. (2003). Russ. J. Org. Chem. 39, 1-28.

Spek, A. L. (2009). Acta Cryst. D65, 148-155.

Stoikov, I. I., Omran, O. A., Solovieva, S. E., Latypov, S. K., Enikeev, K. M., Gubaidullin, A. T., Antipin, I. S. \& Konovalov, A. I. (2003). Tetrahedron, 59, 1469-1476. 


\section{supporting information}

Acta Cryst. (2015). E71, o830-o831 [https://doi.org/10.1107/S2056989015018617]

Crystal structure of the inclusion complex 25-benzoylmethoxy-5,11,17,23-tetratert-butyl-26,27,28-trihydroxy-2,8,14,20-tetrathiacalix[4]arene-tetraethylammonium chloride (1/1)

Mehmet Akkurt, Jerry P. Jasinski, Shaaban K. Mohamed, Omran A. Omran and Mustafa R. Albayati

\section{S1. Comment}

Calixarenes have been found to be an outstanding platform for creating attractive host molecules and have prominent host-guest recognition ability towards different ions. These macrocylic have been used for catalysis, molecular recognition or ion separtion and sensors (Gutsche, 1998; Shokova \& Kovalev, 2003; Stoikov et al., 2003). Since the discovery of $p$-tert-butylthiacalixarene in 1997 as a calixarene analogy with some additional features because of sulfur bridging atom in its skeleton structure, it attracts much attention in research rather than the classical calixarenes and exhibits a wide range of applications in supramolecular chemistry (Iki \& Miyano, 2001; Morohashi et al., 2002;

Desroches et al., 2003; Odo et al., 2000; Odo et al., 2001; Ben Ali et al., 2001; Agrawal \& Pancholi, 2007; Higuchi et al., 2000; Omran \& Antipin, 2014). Many calixarene derivatives are being used in both environmental and biomedical monitoring (McMahon et al., 2003). Based on these findings we report here the synthesis and crystal structure of the title compound.

Two crystallographically independent molecules (A and B) in the asymmetric unit are shown in Figs 1 and 2, respectively, and together with two tetraethylammonium chloride molecules in Fig. 3. The bond lengths and bond angles of molecules $\mathrm{A}$ and $\mathrm{B}$ are within the normal ranges.

In the crystal structure, molecules are linked by $\mathrm{C}-\mathrm{H} \cdots \mathrm{O}, \mathrm{C}-\mathrm{H} \cdots \mathrm{S}, \mathrm{C}-\mathrm{H} \cdots \mathrm{Cl}, \mathrm{O}-\mathrm{H} \cdots \mathrm{O}, \mathrm{O}-\mathrm{H} \cdots \mathrm{S}$ and $\mathrm{O}-\mathrm{H} \cdots \mathrm{Cl}$ interactions, forming a three-dimensional network (Table 1). These lead to the formation of channels running parallel to the $a$-axis direction (Fig. 4).

\section{S2. Experimental}

A mixture of p-tert-butylthiacalix[4]arene $1 \mathrm{~g}(1.38 \mathrm{mmol})$, anhydrous $\mathrm{K}_{2} \mathrm{CO}_{3} 5.0 \mathrm{~g}$, TEAB $0.4 \mathrm{~g}$ and phenacyl bromide $0.28-0.55 \mathrm{~g}(1.38-2.76 \mathrm{mmol})$ in $50 \mathrm{ml}$ benzene was heated at $373 \mathrm{~K}$ for $48 \mathrm{~h}$. the mixture was filtered and the benzene layer was evaporated to dryness. The solid residue was washed by diluted hydrochloric acid solution, extracted with methylene chloride. The methylene chloride layer was dried over magnesium sulfate then filtered and concentrated. The residue was treated with methyl alcohol to deposit a white solid product. The product was filtered off, dried under vacuum and recrystalized from a mixture of methylene chloride/methanol $(1 v / 1 v)$ to give colourless crystals suitable for X-ray diffraction in $90 \%$ yield. 


\section{S3. Refinement}

All $\mathrm{H}$ atoms were positioned geometrically and constrained to ride on their parent atoms $(\mathrm{C}-\mathrm{H}=0.93,0.96$ and $0.97 \AA$, $\mathrm{O}-\mathrm{H}=0.82 \AA) . U_{\text {iso }}(\mathrm{H})$ values were set to a multiple of $U_{\mathrm{eq}}(\mathrm{C}, O)$ with 1.5 for $\mathrm{CH}_{3}$ and $\mathrm{OH}$, and 1.2 for $\mathrm{CH}$ and $\mathrm{CH}_{2}$. The (3 0 1), (4 0 8), (3 0 6), (-21 0 5), (1 4 0), (2 0 4), (-4 -9 22), (-4 -3 4), (1 0 8), (-12 5 3), (-2 0 10), (1 2 1), (-6 -3 6 ), $\left(\begin{array}{ll}-4 & 3\end{array}\right),\left(\begin{array}{lll}1 & -4 & 0\end{array}\right),\left(\begin{array}{lll}0 & 1 & 3\end{array}\right),\left(\begin{array}{lll}3 & 0 & 0\end{array}\right),\left(\begin{array}{lll}6 & 3 & 8\end{array}\right)$ and $\left(\begin{array}{lll}1 & 0 & 1\end{array}\right)$ reflections were omitted owing to very bad agreement.

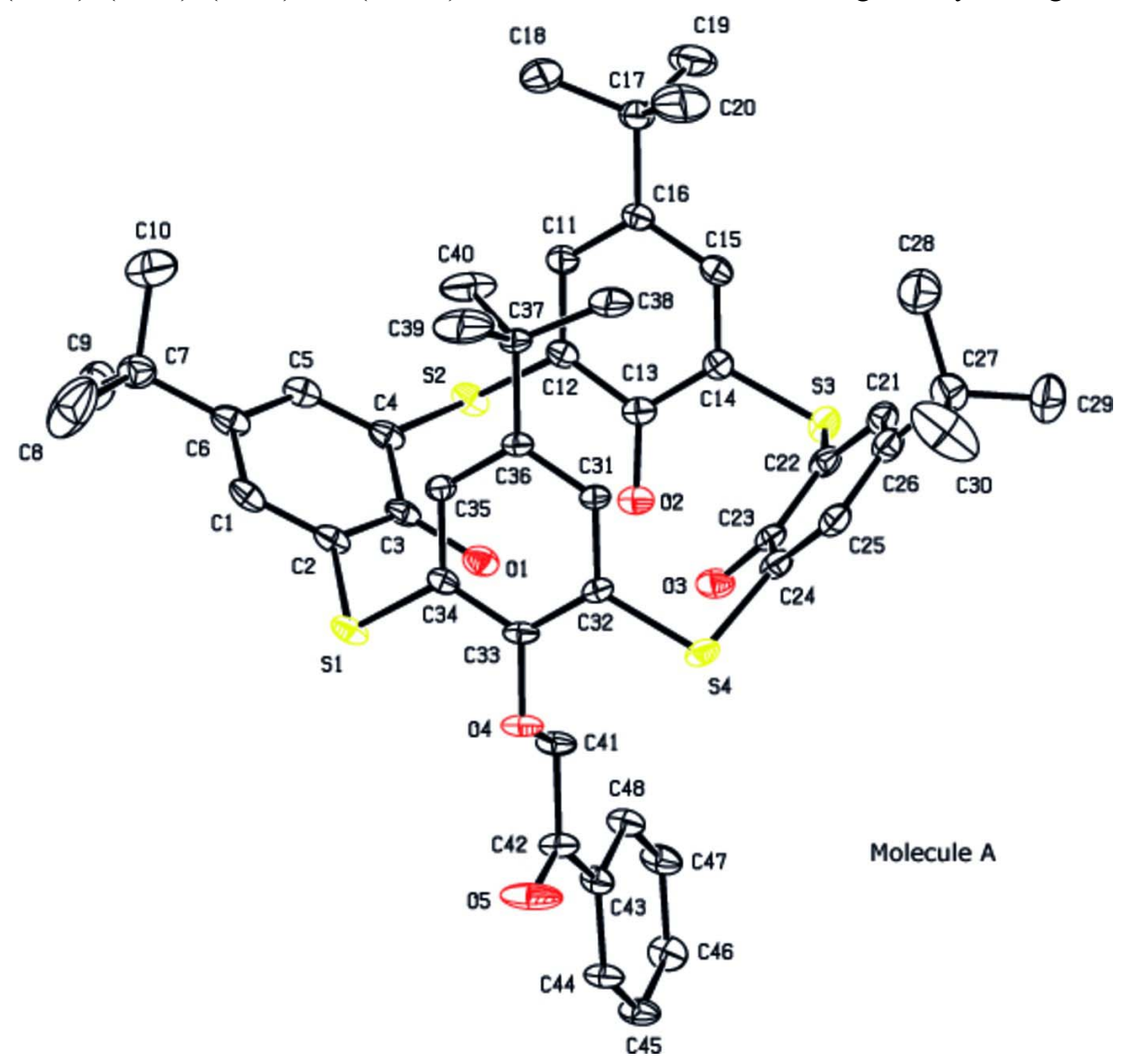

Figure 1

View of the molecule A of two molecules in the asymmetric unit with the atom-numbering scheme. Displacement ellipsoids for non- $\mathrm{H}$ atoms are drawn at the $30 \%$ probability level. All $\mathrm{H}$ atoms are omitted for clarity. 


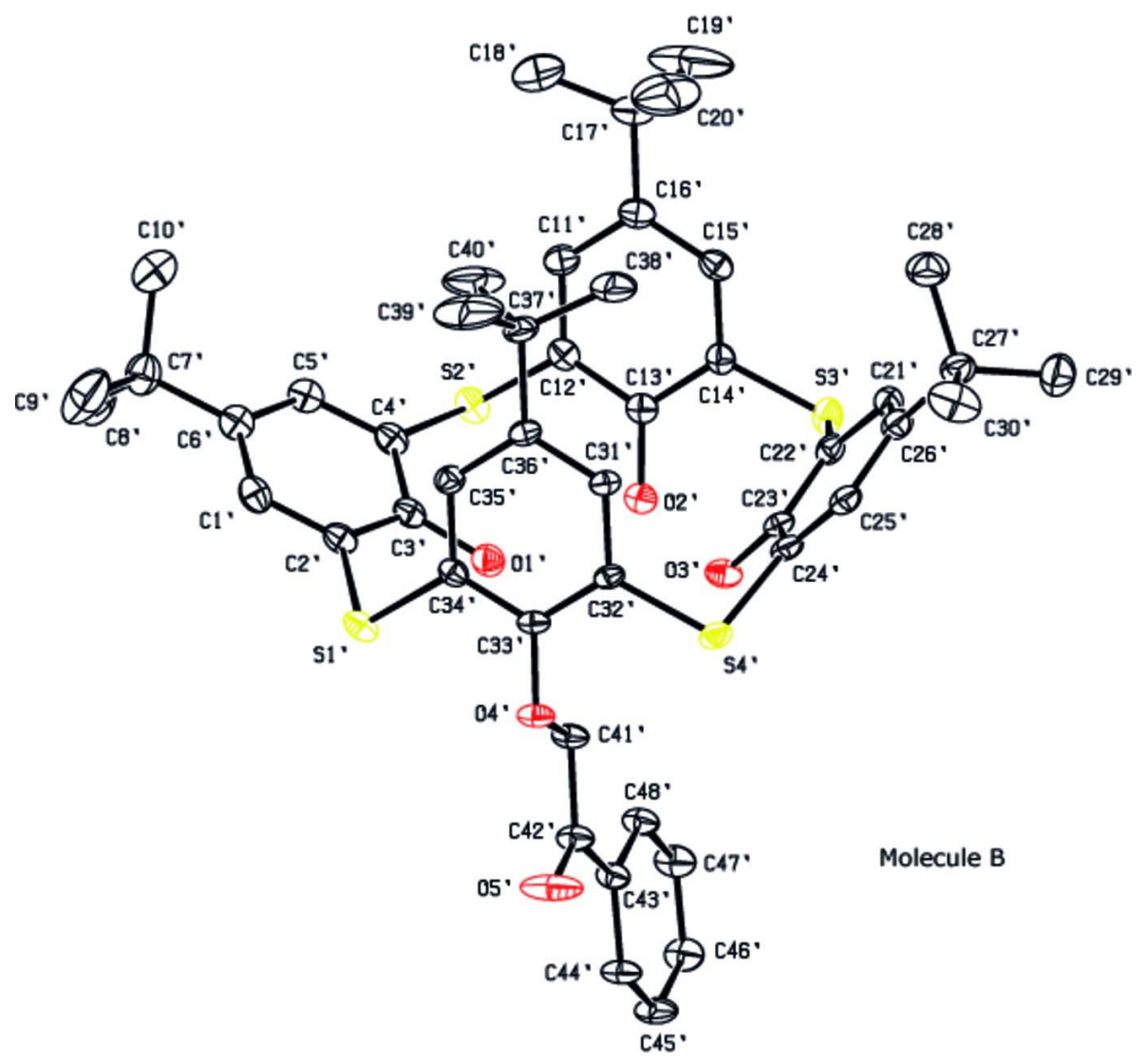

\section{Figure 2}

View of the molecule B of two molecules in the asymmetric unit with the atom-numbering scheme. Displacement ellipsoids for non- $\mathrm{H}$ atoms are drawn at the $30 \%$ probability level. All $\mathrm{H}$ atoms are omitted for clarity.

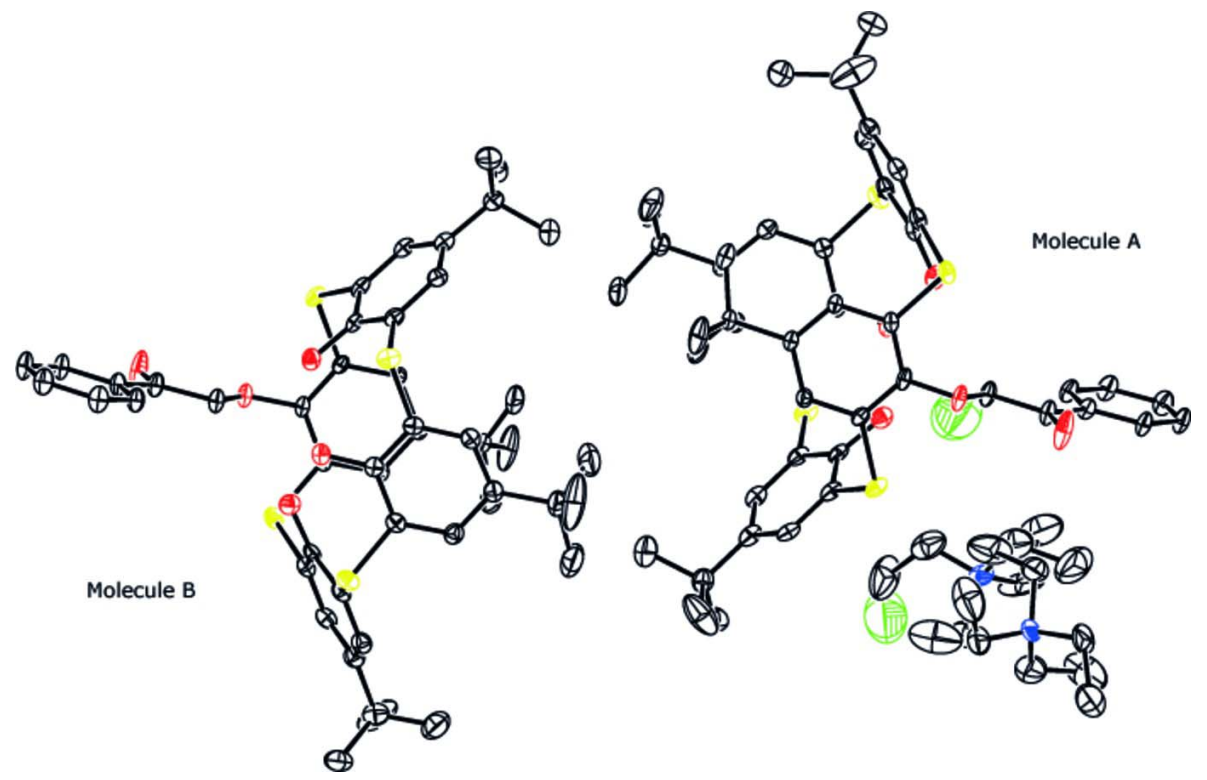

Figure 3

View of two molecules (A and B) with two solvent molecules in the asymmetric unit with the atom numbering scheme. Displacement ellipsoids for non-H atoms are drawn at the $30 \%$ probability level. All $\mathrm{H}$ atoms are omitted for clarity. 


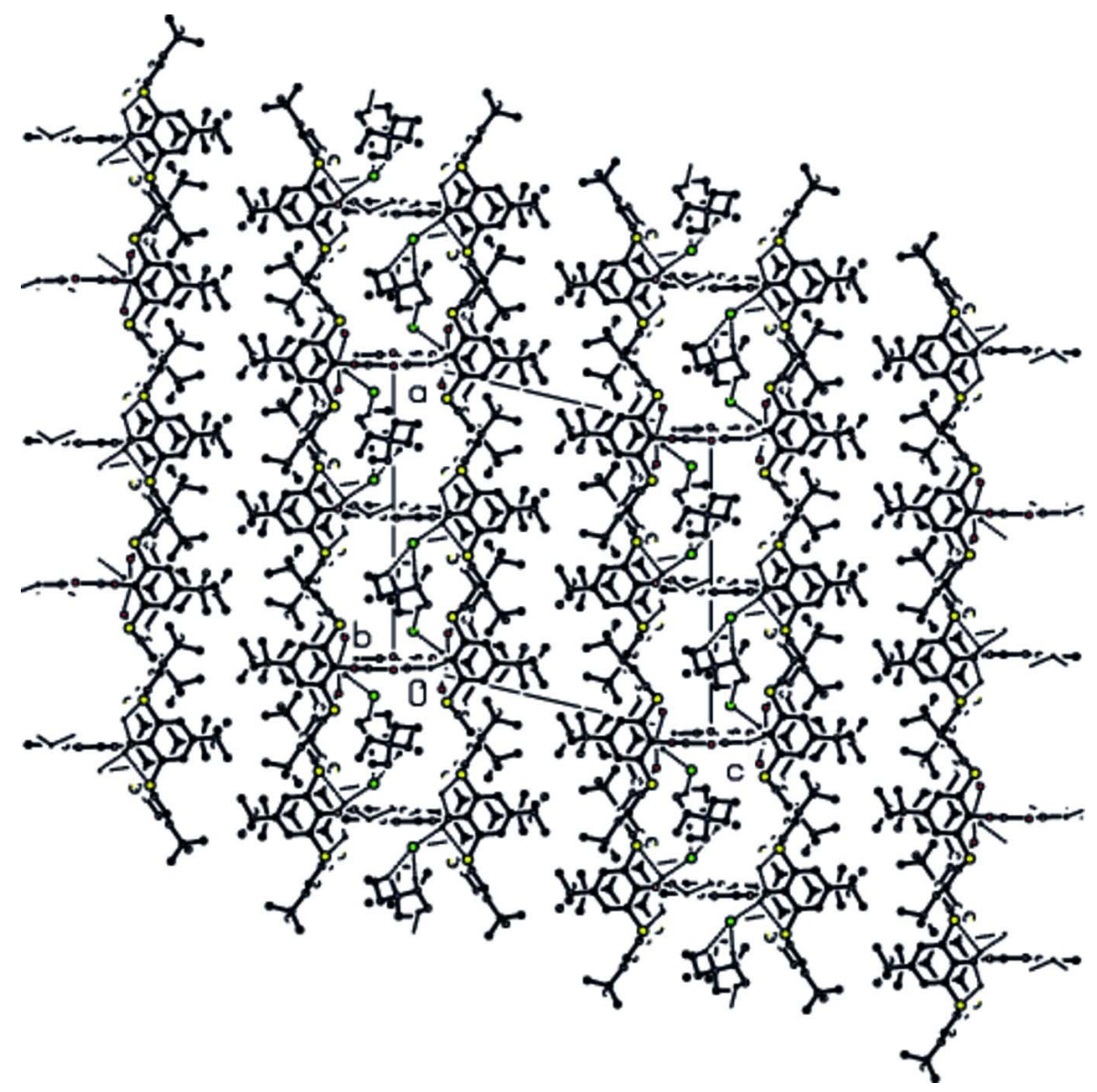

Figure 4

A view of the molecular packing down $b$ axis. $\mathrm{H}$ atoms not involved in $\mathrm{H}$ bonding are omitted for clarity.

25-Benzoylmethoxy-5,11,17,23-tetra-tert-butyl-26,27,28-trihydroxy-2,8,14,20-tetrathiacalix[4]arenetetraethylammonium chloride $(1 / 1)$

Crystal data

$\mathrm{C}_{48} \mathrm{H}_{54} \mathrm{O}_{5} \mathrm{~S}_{4} \cdot \mathrm{C}_{8} \mathrm{H}_{20} \mathrm{~N}^{+} \cdot \mathrm{Cl}^{-}$

$M_{r}=1004.85$

Monoclinic, $P 2_{1}$

Hall symbol: $\mathrm{P} 2 \mathrm{yb}$

$a=19.4338(6) \AA$

$b=14.2147(3) \AA$

$c=20.8631$ (6) $\AA$

$\beta=103.184(3)^{\circ}$

$V=5611.4(3) \AA^{3}$

$Z=4$

\section{Data collection}

Agilent Xcalibur, Eos, Gemini diffractometer

Radiation source: Enhance (Mo) X-ray Source

Graphite monochromator

Detector resolution: 16.0416 pixels $\mathrm{mm}^{-1}$
$F(000)=2152$

$D_{\mathrm{x}}=1.189 \mathrm{Mg} \mathrm{m}^{-3}$

Mo $K \alpha$ radiation, $\lambda=0.71073 \AA$

Cell parameters from 9238 reflections

$\theta=3.2-31.7^{\circ}$

$\mu=0.26 \mathrm{~mm}^{-1}$

$T=293 \mathrm{~K}$

Prism, colourless

$0.42 \times 0.38 \times 0.22 \mathrm{~mm}$

$\omega$ scans

Absorption correction: multi-scan

(CrysAlis PRO; Agilent, 2014)

$T_{\min }=0.922, T_{\max }=1.000$

40706 measured reflections 
25992 independent reflections

18033 reflections with $I>2 \sigma(I)$

$R_{\text {int }}=0.035$

$\theta_{\max }=32.8^{\circ}, \theta_{\min }=3.1^{\circ}$

\section{Refinement}

Refinement on $F^{2}$

Least-squares matrix: full

$R\left[F^{2}>2 \sigma\left(F^{2}\right)\right]=0.079$

$w R\left(F^{2}\right)=0.241$

$S=1.05$

25992 reflections

1208 parameters

8 restraints

$$
\begin{aligned}
& h=-27 \rightarrow 27 \\
& k=-19 \rightarrow 21 \\
& l=-31 \rightarrow 31
\end{aligned}
$$

Hydrogen site location: inferred from neighbouring sites

$\mathrm{H}$-atom parameters constrained

$w=1 /\left[\sigma^{2}\left(F_{\mathrm{o}}^{2}\right)+(0.1278 P)^{2}+2.7781 P\right]$

where $P=\left(F_{\mathrm{o}}^{2}+2 F_{\mathrm{c}}^{2}\right) / 3$

$(\Delta / \sigma)_{\max }<0.001$

$\Delta \rho_{\max }=0.98$ e $\AA^{-3}$

$\Delta \rho_{\min }=-0.88$ e $\AA^{-3}$

Absolute structure: Flack (1983)

Absolute structure parameter: 0.43 (8)

\section{Special details}

Geometry. Bond distances, angles etc. have been calculated using the rounded fractional coordinates. All su's are estimated from the variances of the (full) variance-covariance matrix. The cell esds are taken into account in the estimation of distances, angles and torsion angles

Refinement. Refinement on $\mathrm{F}^{2}$ for ALL reflections except those flagged by the user for potential systematic errors. Weighted R-factors $w R$ and all goodnesses of fit $\mathrm{S}$ are based on $\mathrm{F}^{2}$, conventional $\mathrm{R}$-factors $\mathrm{R}$ are based on $\mathrm{F}$, with $\mathrm{F}$ set to zero for negative $\mathrm{F}^{2}$. The observed criterion of $\mathrm{F}^{2}>2 \operatorname{sigma}\left(\mathrm{F}^{2}\right)$ is used only for calculating -R-factor-obs etc. and is not relevant to the choice of reflections for refinement. R-factors based on $\mathrm{F}^{2}$ are statistically about twice as large as those

\begin{tabular}{|c|c|c|c|c|}
\hline & $x$ & $y$ & $z$ & $U_{\text {iso }} * / U_{\text {eq }}$ \\
\hline $\mathrm{S} 1$ & $0.80181(8)$ & $0.70396(10)$ & $0.81392(7)$ & $0.0378(4)$ \\
\hline $\mathrm{S} 2$ & $0.79798(8)$ & $0.32086(9)$ & $0.76697(7)$ & $0.0375(4)$ \\
\hline S3 & $1.08437(7)$ & $0.32100(10)$ & $0.78599(7)$ & 0.0386 \\
\hline $\mathrm{S} 4$ & $1.08698(7)$ & $0.70419(9)$ & $0.83087(6)$ & $0.0352(3)$ \\
\hline $\mathrm{S} 1^{\prime}$ & $0.39116(7)$ & $0.32712(10)$ & $0.18651(6)$ & 0.0364 \\
\hline $\mathrm{S} 2^{\prime}$ & $0.41163(7)$ & $0.70921(10)$ & $0.23426(7)$ & $0.0376(4)$ \\
\hline S3' & $0.68815(7)$ & $0.70828(10)$ & $0.21467(7)$ & $0.0371(3)$ \\
\hline $\mathrm{S} 4^{\prime}$ & $0.66803(7)$ & $0.32534(9)$ & $0.16843(6)$ & $0.0336(3)$ \\
\hline $\mathrm{Cl1}$ & $0.5890(5)$ & $0.1924(8)$ & $0.9383(4)$ & $0.272(5)$ \\
\hline $\mathrm{O} 1$ & $0.8589(2)$ & $0.5115(3)$ & 0.82787 (19) & $0.0398(11)$ \\
\hline $\mathrm{O} 2$ & $0.9501(2)$ & $0.3800(3)$ & $0.82638(16)$ & $0.0332(10)$ \\
\hline $\mathrm{O} 3$ & $1.0475(2)$ & $0.5057(3)$ & $0.84746(18)$ & $0.0393(11)$ \\
\hline $\mathrm{O} 4$ & $0.9539(2)$ & $0.7408(2)$ & $0.87704(15)$ & $0.0354(9)$ \\
\hline O5 & $0.9786(4)$ & $0.7796(3)$ & 1.00075 (19) & $0.071(2)$ \\
\hline $\mathrm{Cl} 2$ & $0.8781(5)$ & $0.3360(8)$ & $0.9383(4)$ & $0.269(5)$ \\
\hline $\mathrm{C} 1$ & 0.6904 & $0.6073(4)$ & 0.7405 & $0.0373(17)$ \\
\hline $\mathrm{O} 1^{\prime}$ & $0.4415(2)$ & $0.5191(3)$ & 0.17254 (19) & $0.0386(11)$ \\
\hline $\mathrm{C} 2$ & 0.7589 & $0.6001(4)$ & $0.7784(3)$ & $0.0345(14)$ \\
\hline $\mathrm{O} 2^{\prime}$ & 0.53388 (19) & $0.6500(2)$ & 0.17442 (16) & $0.0316(10)$ \\
\hline $\mathrm{C} 3$ & $0.7947(3)$ & $0.5140(4)$ & $0.7881(2)$ & $0.0323(14)$ \\
\hline O3' & $0.6206(2)$ & 0.5239 & $0.15314(17)$ & $0.0370(11)$ \\
\hline
\end{tabular}
based on F, and R-factors based on ALL data will be even larger.

Fractional atomic coordinates and isotropic or equivalent isotropic displacement parameters $\left(\AA^{2}\right)$ 


\begin{tabular}{|c|c|c|c|c|}
\hline $\mathrm{C} 4$ & $0.7594(3)$ & $0.4353(4)$ & $0.7545(2)$ & $0.0336(14)$ \\
\hline O4' & $0.5115(2)$ & $0.2898(2)$ & $0.12228(15)$ & $0.0319(9)$ \\
\hline $\mathrm{C} 5$ & 0.6911 (3) & $0.4432(4)$ & $0.7169(3)$ & $0.0367(16)$ \\
\hline O5' & 0.4777 (4) & $0.2509(3)$ & $-0.00141(19)$ & $0.072(2)$ \\
\hline $\mathrm{C} 6$ & $0.6547(3)$ & $0.5280(4)$ & 0.7093 & $0.0399(17)$ \\
\hline $\mathrm{C} 7$ & $0.5784(4)$ & $0.5339(5)$ & $0.6676(3)$ & $0.0467(17)$ \\
\hline C8 & $0.5516(6)$ & $0.6335(8)$ & $0.6604(7)$ & $0.119(5)$ \\
\hline $\mathrm{C} 9$ & $0.5321(4)$ & $0.4741(8)$ & $0.7010(4)$ & $0.075(3)$ \\
\hline $\mathrm{C} 10$ & $0.5757(6)$ & $0.4932(11)$ & $0.5992(5)$ & $0.105(5)$ \\
\hline C11 & $0.8604(3)$ & $0.2973(4)$ & $0.6625(2)$ & $0.0331(12)$ \\
\hline $\mathrm{C} 12$ & 0.8709 (2) & $0.3240(4)$ & $0.7288(2)$ & 0.0283 \\
\hline $\mathrm{C} 13$ & $0.9400(3)$ & $0.3436(3)$ & $0.7676(2)$ & $0.0290(14)$ \\
\hline C14 & $0.9963(2)$ & $0.3213(3)$ & $0.7379(2)$ & $0.0292(11)$ \\
\hline $\mathrm{C} 15$ & $0.9840(3)$ & $0.2946(3)$ & $0.6716(2)$ & $0.0318(14)$ \\
\hline $\mathrm{C} 16$ & $0.9163(3)$ & $0.2858(4)$ & $0.6318(2)$ & $0.0311(12)$ \\
\hline $\mathrm{C} 17$ & $0.9045(3)$ & $0.2603(4)$ & $0.5588(2)$ & $0.0402(16)$ \\
\hline $\mathrm{C} 18$ & $0.8299(5)$ & $0.2777(9)$ & $0.5210(3)$ & $0.091(4)$ \\
\hline C19 & $0.9228(5)$ & $0.1576(6)$ & $0.5523(4)$ & $0.069(3)$ \\
\hline $\mathrm{C} 20$ & $0.9535(5)$ & $0.3218(8)$ & $0.5270(3)$ & 0.083 \\
\hline $\mathrm{C} 21$ & $1.1611(3)$ & $0.4491(4)$ & $0.7343(3)$ & $0.0384(17)$ \\
\hline $\mathrm{C} 22$ & $1.1141(3)$ & $0.4370(4)$ & $0.7740(2)$ & $0.0349(14)$ \\
\hline $\mathrm{C} 23$ & $1.0916(3)$ & $0.5131(4)$ & $0.8073(2)$ & $0.0301(11)$ \\
\hline $\mathrm{C} 24$ & $1.1186(3)$ & $0.6025(4)$ & $0.7965(2)$ & $0.0351(12)$ \\
\hline $\mathrm{C} 25$ & $1.1662(3)$ & $0.6138(4)$ & 0.7564 & $0.0375(16)$ \\
\hline $\mathrm{C} 26$ & $1.1876(3)$ & $0.5385(4)$ & $0.7232(3)$ & $0.0394(17)$ \\
\hline $\mathrm{C} 27$ & $1.2369(4)$ & $0.5485(5)$ & 0.6768 & $0.0490(19)$ \\
\hline $\mathrm{C} 28$ & $1.1999(5)$ & $0.5128(8)$ & $0.6084(4)$ & $0.077(3)$ \\
\hline $\mathrm{C} 29$ & $1.3035(4)$ & $0.4928(8)$ & $0.7018(5)$ & $0.077(3)$ \\
\hline $\mathrm{C} 30$ & $1.2592(8)$ & $0.6506(7)$ & $0.6711(7)$ & $0.114(6)$ \\
\hline C31 & $0.9866(2)$ & $0.6758(3)$ & $0.7159(2)$ & $0.0258(11)$ \\
\hline $\mathrm{C} 32$ & $0.9990(2)$ & $0.7007(3)$ & $0.78212(19)$ & $0.0243(10)$ \\
\hline $\mathrm{C} 33$ & $0.9425(3)$ & $0.7144(3)$ & $0.81181(19)$ & $0.0277(13)$ \\
\hline C34 & $0.8743(3)$ & $0.7014(4)$ & $0.7749(2)$ & 0.0278 \\
\hline C35 & $0.8624(3)$ & $0.6776(4)$ & $0.7084(2)$ & $0.0298(11)$ \\
\hline $\mathrm{C} 36$ & $0.9189(3)$ & $0.6639(3)$ & $0.6779(2)$ & $0.0271(11)$ \\
\hline $\mathrm{C} 37$ & $0.9057(3)$ & $0.6351(4)$ & $0.6048(2)$ & 0.0340 \\
\hline $\mathrm{C} 38$ & $0.9711(4)$ & $0.5952(9)$ & 0.5879 & $0.084(4)$ \\
\hline C39 & $0.8830(6)$ & $0.7202(7)$ & $0.5621(3)$ & 0.079 (3) \\
\hline $\mathrm{C} 40$ & $0.8473(5)$ & $0.5604(7)$ & $0.5886(3)$ & $0.070(3)$ \\
\hline $\mathrm{C} 41$ & $0.9633(3)$ & $0.6610(3)$ & $0.9205(2)$ & 0.0348 \\
\hline $\mathrm{C} 42$ & $0.9788(3)$ & $0.6964(4)$ & $0.9898(2)$ & $0.0351(13)$ \\
\hline C43 & 0.9947 (3) & 0.6261 (3) & $1.0436(2)$ & $0.0312(14)$ \\
\hline $\mathrm{C} 44$ & $1.0124(3)$ & $0.6590(4)$ & $1.1089(2)$ & $0.0360(14)$ \\
\hline $\mathrm{C} 45$ & $1.0281(4)$ & $0.5972(4)$ & $1.1603(2)$ & $0.0417(18)$ \\
\hline $\mathrm{C} 46$ & $1.0254(4)$ & $0.5011(4)$ & $1.1481(3)$ & 0.0433 \\
\hline $\mathrm{C} 47$ & $1.0081(4)$ & $0.4679(4)$ & $1.0844(3)$ & $0.0424(16)$ \\
\hline $\mathrm{C} 48$ & $0.9926(3)$ & $0.5301(4)$ & $1.0321(2)$ & 0.0365 (14) \\
\hline $\mathrm{C} 1^{\prime}$ & 0.3177 (3) & $0.4237(4)$ & $0.2627(3)$ & $0.0391(16)$ \\
\hline
\end{tabular}




\begin{tabular}{|c|c|c|c|c|}
\hline $\mathrm{C} 2^{\prime}$ & $0.3667(3)$ & $0.4301(4)$ & $0.2231(3)$ & $0.0346(14)$ \\
\hline C3' & $0.3975(3)$ & $0.5166(4)$ & $0.2133(2)$ & $0.0314(12)$ \\
\hline $\mathrm{C} 4^{\prime}$ & $0.3794(3)$ & $0.5954(4)$ & 0.2468 & $0.0344(12)$ \\
\hline $\mathrm{C} 5^{\prime}$ & $0.3303(3)$ & $0.5871(4)$ & $0.2864(3)$ & 0.0383 \\
\hline $\mathrm{C} 6^{\prime}$ & 0.2988 & $0.5021(4)$ & $0.2947(3)$ & $0.0400(17)$ \\
\hline$C 7^{\prime}$ & 0.2441 (4) & $0.4977(5)$ & $0.3373(4)$ & $0.052(2)$ \\
\hline $\mathrm{C} 8^{\prime}$ & $0.2295(7)$ & $0.3968(7)$ & $0.3541(6)$ & $0.102(5)$ \\
\hline $\mathrm{C} 9^{\prime}$ & $0.1775(4)$ & $0.5495(8)$ & $0.3007(4)$ & $0.073(3)$ \\
\hline $\mathrm{C} 10^{\prime}$ & $0.2704(5)$ & $0.5479(8)$ & $0.4020(4)$ & $0.082(3)$ \\
\hline C11' & $0.5250(3)$ & $0.7316(4)$ & $0.3387(3)$ & $0.0363(16)$ \\
\hline C12' & $0.5031(3)$ & $0.7055(4)$ & $0.2726(2)$ & 0.0312 \\
\hline C13' & 0.5528 & $0.6864(3)$ & $0.2336(2)$ & $0.0285(12)$ \\
\hline C14' & $0.6242(2)$ & $0.7080(4)$ & $0.2634(2)$ & 0.0298 \\
\hline C15' & 0.6439 (3) & $0.7340(4)$ & $0.3291(3)$ & $0.0339(12)$ \\
\hline C16' & $0.5966(3)$ & $0.7429(4)$ & $0.3697(3)$ & 0.0368 \\
\hline $\mathrm{C} 17^{\prime}$ & $0.6204(4)$ & $0.7690(5)$ & $0.4425(3)$ & $0.0492(18)$ \\
\hline C18' & $0.5621(6)$ & $0.7607(10)$ & $0.4780(4)$ & $0.096(4)$ \\
\hline C19' & $0.6451(10)$ & $0.8685(8)$ & $0.4470(5)$ & $0.149(7)$ \\
\hline $\mathrm{C} 20^{\prime}$ & $0.6783(6)$ & $0.6990(11)$ & $0.4766(4)$ & $0.116(5)$ \\
\hline $\mathrm{C} 21^{\prime}$ & $0.7901(3)$ & $0.5798(4)$ & $0.2654(3)$ & $0.0337(14)$ \\
\hline $\mathrm{C} 22^{\prime}$ & $0.7226(3)$ & $0.5926(4)$ & $0.2258(2)$ & $0.0311(12)$ \\
\hline $\mathrm{C} 23^{\prime}$ & $0.6846(3)$ & $0.5165(4)$ & $0.1927(2)$ & 0.0283 \\
\hline $\mathrm{C} 24^{\prime}$ & 0.7168 & $0.4263(4)$ & $0.2027(2)$ & $0.0321(14)$ \\
\hline $\mathrm{C} 25^{\prime}$ & $0.7840(3)$ & $0.4154(4)$ & $0.2423(2)$ & $0.0332(14)$ \\
\hline $\mathrm{C} 26^{\prime}$ & $0.8225(3)$ & $0.4916(4)$ & $0.2757(3)$ & $0.0353(16)$ \\
\hline $\mathrm{C} 27^{\prime}$ & 0.8948 & $0.4798(4)$ & $0.3216(3)$ & $0.0407(17)$ \\
\hline $\mathrm{C} 28^{\prime}$ & $0.8932(4)$ & $0.5136(6)$ & 0.3901 & $0.057(2)$ \\
\hline $\mathrm{C} 29^{\prime}$ & 0.9488 (4) & $0.5381(7)$ & $0.2947(4)$ & 0.063 \\
\hline $\mathrm{C} 30^{\prime}$ & $0.9196(5)$ & $0.3764(6)$ & $0.3288(4)$ & $0.067(3)$ \\
\hline C31' & $0.6250(3)$ & $0.3515(3)$ & $0.2838(2)$ & $0.0272(11)$ \\
\hline C $32^{\prime}$ & $0.6039(2)$ & $0.3282(3)$ & $0.2173(2)$ & 0.0254 \\
\hline C33' & $0.5331(2)$ & $0.3155(3)$ & $0.18780(19)$ & $0.0248(10)$ \\
\hline C $34^{\prime}$ & $0.4828(2)$ & $0.3283(4)$ & $0.2256(2)$ & $0.0277(11)$ \\
\hline $\mathrm{C} 35^{\prime}$ & $0.5043(3)$ & $0.3506(4)$ & $0.2918(2)$ & 0.0308 \\
\hline $\mathrm{C} 36^{\prime}$ & $0.5755(3)$ & $0.3630(4)$ & $0.3224(2)$ & $0.0292(11)$ \\
\hline C37' & 0.5979 (3) & $0.3920(4)$ & $0.3949(2)$ & $0.0369(15)$ \\
\hline C38' & $0.6710(5)$ & $0.4311(9)$ & $0.4127(4)$ & 0.089 (4) \\
\hline C39' & $0.5951(8)$ & $0.3079(8)$ & $0.4373(3)$ & $0.106(5)$ \\
\hline $\mathrm{C} 40^{\prime}$ & $0.5481(5)$ & $0.4694(9)$ & $0.4094(4)$ & $0.098(4)$ \\
\hline $\mathrm{C} 41^{\prime}$ & $0.5008(3)$ & $0.3695(3)$ & $0.0793(2)$ & $0.0317(13)$ \\
\hline $\mathrm{C} 42^{\prime}$ & $0.4819(3)$ & $0.3344(4)$ & $0.0099(2)$ & $0.0343(14)$ \\
\hline $\mathrm{C} 43^{\prime}$ & $0.4714(3)$ & $0.4048(4)$ & $-0.0438(2)$ & $0.0312(14)$ \\
\hline C44' & 0.4567 (3) & $0.3710(4)$ & $-0.1090(2)$ & $0.0372(14)$ \\
\hline $\mathrm{C} 45^{\prime}$ & $0.4467(4)$ & $0.4330(4)$ & $-0.1606(2)$ & $0.0439(18)$ \\
\hline $\mathrm{C} 46^{\prime}$ & $0.4517(4)$ & $0.5298(4)$ & $-0.1485(3)$ & $0.0426(18)$ \\
\hline $\mathrm{C} 47^{\prime}$ & $0.4655(3)$ & $0.5623(4)$ & $-0.0849(3)$ & $0.0427(18)$ \\
\hline $\mathrm{C} 48^{\prime}$ & $0.4762(3)$ & $0.5000(4)$ & $-0.0325(2)$ & $0.0356(14)$ \\
\hline N1 & $0.7316(3)$ & $0.5160(5)$ & $0.9884(3)$ & $0.0496(17)$ \\
\hline
\end{tabular}




\begin{tabular}{|c|c|c|c|c|}
\hline C101 & $0.6644(5)$ & $0.5741(8)$ & $0.9824(6)$ & $0.091(4)$ \\
\hline C102 & $0.6541(8)$ & $0.6476(10)$ & $0.9349(10)$ & $0.148(8)$ \\
\hline $\mathrm{C} 103$ & $0.7320(7)$ & $0.4724(9)$ & $0.9252(5)$ & 0.098 (4) \\
\hline C104 & $0.6633(9)$ & 0.4157 (13) & 0.8927 (6) & $0.148(8)$ \\
\hline $\mathrm{C} 105$ & $0.7357(8)$ & 0.4551 (14) & $1.0466(6)$ & $0.179(8)$ \\
\hline C106 & $0.7984(10)$ & 0.3894 (17) & $1.0618(7)$ & $0.200(12)$ \\
\hline C107 & $0.7949(7)$ & $0.5819(12)$ & $1.0075(7)$ & 0.119 (6) \\
\hline C108 & 0.8067 (9) & $0.6162(19)$ & 1.0767 (9) & $0.202(12)$ \\
\hline $\mathrm{N} 2$ & $0.7630(3)$ & $1.0163(4)$ & 0.9884 (3) & $0.0482(16)$ \\
\hline C201 & $0.8276(4)$ & $0.9585(7)$ & $0.9832(5)$ & $0.073(3)$ \\
\hline C202 & $0.8157(7)$ & $0.8812(9)$ & 0.9367 (9) & $0.137(7)$ \\
\hline C203 & $0.7296(5)$ & $1.0612(8)$ & $0.9248(4)$ & $0.080(3)$ \\
\hline C204 & $0.7792(8)$ & 1.1205 (12) & $0.8923(6)$ & $0.133(6)$ \\
\hline $\mathrm{C} 205$ & $0.7912(5)$ & $1.0878(9)$ & $1.0404(5)$ & $0.107(4)$ \\
\hline C206 & $0.7330(8)$ & $1.1501(13)$ & $1.0580(7)$ & $0.168(8)$ \\
\hline $\mathrm{C} 207$ & $0.7100(6)$ & $0.9540(11)$ & $1.0089(6)$ & $0.103(5)$ \\
\hline C208 & $0.7315(7)$ & 0.9154 (16) & $1.0784(8)$ & $0.173(8)$ \\
\hline H1 & 0.66790 & 0.66540 & 0.73570 & $0.0450 *$ \\
\hline $\mathrm{H} 1 \mathrm{O}$ & 0.87440 & 0.45770 & 0.82950 & $0.0600 *$ \\
\hline $\mathrm{H} 2 \mathrm{O}$ & 0.92590 & 0.35180 & 0.84760 & $0.0500^{*}$ \\
\hline $\mathrm{H} 3 \mathrm{O}$ & 1.03590 & 0.45060 & 0.84950 & $0.0590^{*}$ \\
\hline H5 & 0.66880 & 0.38990 & 0.69600 & $0.0440^{*}$ \\
\hline H8A & 0.55340 & 0.65960 & 0.70320 & $0.1780^{*}$ \\
\hline H8B & 0.50370 & 0.63410 & 0.63510 & $0.1780^{*}$ \\
\hline $\mathrm{H} 8 \mathrm{C}$ & 0.58070 & 0.67030 & 0.63840 & $0.1780^{*}$ \\
\hline H9A & 0.53270 & 0.49950 & 0.74380 & $0.1120^{*}$ \\
\hline H9B & 0.54980 & 0.41080 & 0.70550 & $0.1120^{*}$ \\
\hline $\mathrm{H} 9 \mathrm{C}$ & 0.48450 & 0.47420 & 0.67490 & $0.1120^{*}$ \\
\hline $\mathrm{H} 10 \mathrm{~A}$ & 0.59310 & 0.42970 & 0.60350 & $0.1580^{*}$ \\
\hline H10B & 0.60460 & 0.53060 & 0.57740 & $0.1580^{*}$ \\
\hline $\mathrm{H} 10 \mathrm{C}$ & 0.52780 & 0.49360 & 0.57390 & $0.1580^{*}$ \\
\hline H11 & 0.81460 & 0.28700 & 0.63830 & $0.0390 *$ \\
\hline H15 & 1.02260 & 0.28220 & 0.65350 & $0.0380^{*}$ \\
\hline $\mathrm{H} 18 \mathrm{~A}$ & 0.81820 & 0.34290 & 0.52460 & $0.1360^{*}$ \\
\hline H18B & 0.79800 & 0.23940 & 0.53870 & $0.1360^{*}$ \\
\hline $\mathrm{H} 18 \mathrm{C}$ & 0.82580 & 0.26210 & 0.47550 & $0.1360^{*}$ \\
\hline H19A & 0.97050 & 0.14630 & 0.57610 & $0.1030^{*}$ \\
\hline H19B & 0.91850 & 0.14270 & 0.50660 & $0.1030^{*}$ \\
\hline H19C & 0.89120 & 0.11880 & 0.56990 & $0.1030^{*}$ \\
\hline $\mathrm{H} 20 \mathrm{~A}$ & 1.00180 & 0.31200 & 0.54980 & $0.1240^{*}$ \\
\hline H20B & 0.94150 & 0.38690 & 0.53000 & $0.1240^{*}$ \\
\hline $\mathrm{H} 20 \mathrm{C}$ & 0.94770 & 0.30470 & 0.48160 & $0.1240^{*}$ \\
\hline $\mathrm{H} 21$ & 1.17580 & 0.39680 & 0.71420 & $0.0460 *$ \\
\hline H25 & 1.18420 & 0.67340 & 0.75170 & $0.0450^{*}$ \\
\hline H28A & 1.23120 & 0.51910 & 0.57910 & $0.1160 *$ \\
\hline $\mathrm{H} 28 \mathrm{~B}$ & 1.15790 & 0.54920 & 0.59210 & $0.1160^{*}$ \\
\hline $\mathrm{H} 28 \mathrm{C}$ & 1.18740 & 0.44790 & 0.61120 & $0.1160^{*}$ \\
\hline H29A & 1.32660 & 0.51480 & 0.74490 & $0.1150^{*}$ \\
\hline
\end{tabular}




\begin{tabular}{|c|c|c|c|c|}
\hline H29B & 1.33460 & 0.50070 & 0.67240 & $0.1150^{*}$ \\
\hline $\mathrm{H} 29 \mathrm{C}$ & 1.29200 & 0.42740 & 0.70410 & $0.1150^{*}$ \\
\hline $\mathrm{H} 30 \mathrm{~A}$ & 1.28240 & 0.67360 & 0.71380 & $0.1710^{*}$ \\
\hline H30B & 1.21820 & 0.68830 & 0.65380 & $0.1710^{*}$ \\
\hline $\mathrm{H} 30 \mathrm{C}$ & 1.29110 & 0.65410 & 0.64220 & $0.1710^{*}$ \\
\hline H31 & 1.02490 & 0.66700 & 0.69680 & $0.0310^{*}$ \\
\hline H35 & 0.81630 & 0.67080 & 0.68390 & $0.0360 *$ \\
\hline H38A & 0.98580 & 0.54010 & 0.61400 & $0.1270 *$ \\
\hline H38B & 1.00810 & 0.64130 & 0.59680 & $0.1270^{*}$ \\
\hline $\mathrm{H} 38 \mathrm{C}$ & 0.96100 & 0.57870 & 0.54210 & $0.1270^{*}$ \\
\hline H39A & 0.91950 & 0.76720 & 0.57170 & $0.1180^{*}$ \\
\hline H39B & 0.84020 & 0.74520 & 0.57090 & $0.1180^{*}$ \\
\hline H39C & 0.87500 & 0.70250 & 0.51660 & $0.1180^{*}$ \\
\hline $\mathrm{H} 40 \mathrm{~A}$ & 0.86120 & 0.50600 & 0.61580 & $0.1050^{*}$ \\
\hline H40B & 0.83990 & 0.54290 & 0.54310 & $0.1050 *$ \\
\hline $\mathrm{H} 40 \mathrm{C}$ & 0.80430 & 0.58550 & 0.59690 & $0.1050 *$ \\
\hline H41A & 1.00220 & 0.62250 & 0.91350 & $0.0420 *$ \\
\hline H41B & 0.92080 & 0.62290 & 0.91180 & $0.0420^{*}$ \\
\hline H44 & 1.01340 & 0.72330 & 1.11710 & $0.0430 *$ \\
\hline $\mathrm{H} 45$ & 1.04070 & 0.61940 & 1.20330 & $0.0500 *$ \\
\hline H46 & 1.03520 & 0.45890 & 1.18310 & $0.0520^{*}$ \\
\hline H47 & 1.00690 & 0.40340 & 1.07660 & $0.0510^{*}$ \\
\hline $\mathrm{H} 48$ & 0.98070 & 0.50740 & 0.98910 & $0.0440 *$ \\
\hline $\mathrm{H} 1^{\prime}$ & 0.29730 & 0.36580 & 0.26770 & $0.0470 *$ \\
\hline H8A' & 0.21250 & 0.36190 & 0.31430 & $0.1520^{*}$ \\
\hline H8B' & 0.19460 & 0.39600 & 0.38000 & $0.1520^{*}$ \\
\hline $\mathrm{H} 8 \mathrm{C}^{\prime}$ & 0.27240 & 0.36860 & 0.37870 & $0.1520 *$ \\
\hline H9A' & 0.15910 & 0.51920 & 0.25920 & $0.1100 *$ \\
\hline H5' & 0.31870 & 0.64020 & 0.30770 & $0.0460 *$ \\
\hline $\mathrm{H}^{\prime} \mathrm{B}^{\prime}$ & 0.18920 & 0.61360 & 0.29330 & $0.1100 *$ \\
\hline $\mathrm{H}^{\prime} \mathrm{C}^{\prime}$ & 0.14270 & 0.54810 & 0.32670 & $0.1100^{*}$ \\
\hline $\mathrm{H} 6 \mathrm{O}$ & 0.45610 & 0.57290 & 0.17070 & $0.0580 *$ \\
\hline $\mathrm{H} 7 \mathrm{O}$ & 0.49830 & 0.67670 & 0.15380 & $0.0470 *$ \\
\hline $\mathrm{H} 8 \mathrm{O}$ & 0.60770 & 0.57900 & 0.15160 & $0.0550 *$ \\
\hline H10D & 0.28040 & 0.61230 & 0.39390 & $0.1230 *$ \\
\hline H10E & 0.31270 & 0.51780 & 0.42610 & $0.1230 *$ \\
\hline $\mathrm{H} 10 \mathrm{~F}$ & 0.23490 & 0.54530 & 0.42730 & $0.1230^{*}$ \\
\hline H11' & 0.49110 & 0.74170 & 0.36300 & $0.0440 *$ \\
\hline H15' & 0.69140 & 0.74610 & 0.34700 & $0.0410 *$ \\
\hline H18D & 0.54540 & 0.69690 & 0.47550 & $0.1440 *$ \\
\hline H18E & 0.52400 & 0.80170 & 0.45800 & $0.1440 *$ \\
\hline $\mathrm{H} 18 \mathrm{~F}$ & 0.57930 & 0.77800 & 0.52330 & $0.1440 *$ \\
\hline H19D & 0.68290 & 0.87510 & 0.42460 & $0.2210^{*}$ \\
\hline H19E & 0.66170 & 0.88560 & 0.49240 & $0.2210^{*}$ \\
\hline $\mathrm{H} 19 \mathrm{~F}$ & 0.60670 & 0.90900 & 0.42680 & $0.2210^{*}$ \\
\hline H20D & 0.65950 & 0.63630 & 0.47240 & $0.1740^{*}$ \\
\hline $\mathrm{H} 20 \mathrm{E}$ & 0.69360 & 0.71480 & 0.52240 & $0.1740^{*}$ \\
\hline $\mathrm{H} 20 \mathrm{~F}$ & 0.71770 & 0.70250 & 0.45610 & $0.1740 *$ \\
\hline
\end{tabular}




\begin{tabular}{|c|c|c|c|c|}
\hline $\mathrm{H} 21^{\prime}$ & 0.81460 & 0.63200 & 0.28580 & $0.0400 *$ \\
\hline $\mathrm{H} 25^{\prime}$ & 0.80430 & 0.35580 & 0.24690 & $0.0390 *$ \\
\hline $\mathrm{H} 28 \mathrm{D}$ & 0.93920 & 0.50600 & 0.41870 & $0.0850 *$ \\
\hline $\mathrm{H} 28 \mathrm{E}$ & 0.85940 & 0.47730 & 0.40670 & $0.0850 *$ \\
\hline $\mathrm{H} 28 \mathrm{~F}$ & 0.88000 & 0.57880 & 0.38840 & $0.0850 *$ \\
\hline H29D & 0.99470 & 0.53140 & 0.32330 & $0.0940 *$ \\
\hline H29E & 0.93510 & 0.60310 & 0.29240 & $0.0940 *$ \\
\hline $\mathrm{H} 29 \mathrm{~F}$ & 0.95010 & 0.51610 & 0.25140 & $0.0940 *$ \\
\hline H30D & 0.96540 & 0.37290 & 0.35820 & $0.1000^{*}$ \\
\hline $\mathrm{H} 30 \mathrm{E}$ & 0.92220 & 0.35210 & 0.28650 & $0.1000^{*}$ \\
\hline $\mathrm{H} 30 \mathrm{~F}$ & 0.88650 & 0.33970 & 0.34620 & $0.1000 *$ \\
\hline $\mathrm{H} 31^{\prime}$ & 0.67280 & 0.35950 & 0.30290 & $0.0330 *$ \\
\hline H35' & 0.47040 & 0.35740 & 0.31650 & $0.0370^{*}$ \\
\hline H38D & 0.67360 & 0.48620 & 0.38670 & $0.1330^{*}$ \\
\hline H38E & 0.70380 & 0.38480 & 0.40420 & 0.1330 * \\
\hline $\mathrm{H} 38 \mathrm{~F}$ & 0.68270 & 0.44740 & 0.45850 & $0.1330 *$ \\
\hline H39D & 0.62640 & 0.26020 & 0.42780 & $0.1590^{*}$ \\
\hline H39E & 0.54770 & 0.28380 & 0.42840 & $0.1590 *$ \\
\hline $\mathrm{H} 39 \mathrm{~F}$ & 0.60950 & 0.32560 & 0.48280 & $0.1590^{*}$ \\
\hline H40D & 0.55090 & 0.52340 & 0.38240 & $0.1470^{*}$ \\
\hline $\mathrm{H} 40 \mathrm{E}$ & 0.56190 & 0.48690 & 0.45500 & $0.1470^{*}$ \\
\hline $\mathrm{H} 40 \mathrm{~F}$ & 0.50040 & 0.44620 & 0.39980 & $0.1470 *$ \\
\hline $\mathrm{H} 41 \mathrm{C}$ & 0.54360 & 0.40710 & 0.08630 & $0.0380^{*}$ \\
\hline H41D & 0.46300 & 0.40860 & 0.08800 & $0.0380^{*}$ \\
\hline H44' & 0.45370 & 0.30660 & -0.11700 & $0.0440 *$ \\
\hline $\mathrm{H} 45^{\prime}$ & 0.43660 & 0.41080 & -0.20360 & $0.0530 *$ \\
\hline $\mathrm{H} 46^{\prime}$ & 0.44580 & 0.57200 & -0.18340 & $0.0510^{*}$ \\
\hline $\mathrm{H} 47^{\prime}$ & 0.46760 & 0.62670 & -0.07690 & $0.0510^{*}$ \\
\hline $\mathrm{H}_{4} 8^{\prime}$ & 0.48660 & 0.52280 & 0.01040 & $0.0430^{*}$ \\
\hline H1QW & 0.76530 & 0.64880 & 1.08260 & $0.3040 *$ \\
\hline H10G & 0.62420 & 0.53190 & 0.97170 & $0.1090 *$ \\
\hline $\mathrm{H} 10 \mathrm{H}$ & 0.66490 & 0.60160 & 1.02510 & $0.1090 *$ \\
\hline H10I & 0.61110 & 0.68050 & 0.93540 & $0.2220 *$ \\
\hline H10J & 0.65110 & 0.62130 & 0.89190 & $0.2220^{*}$ \\
\hline H10K & 0.69320 & 0.69060 & 0.94520 & $0.2220^{*}$ \\
\hline H10L & 0.77210 & 0.43010 & 0.93120 & 0.1180 * \\
\hline H10M & 0.73890 & 0.52130 & 0.89490 & $0.1180 *$ \\
\hline $\mathrm{H} 10 \mathrm{~N}$ & 0.66840 & 0.38950 & 0.85160 & $0.2220^{*}$ \\
\hline $\mathrm{H} 10 \mathrm{O}$ & 0.62330 & 0.45720 & 0.88490 & $0.2220^{*}$ \\
\hline H10P & 0.65630 & 0.36590 & 0.92160 & $0.2220 *$ \\
\hline H10Q & 0.84640 & 0.65820 & 1.08570 & $0.3040 *$ \\
\hline H10R & 0.69280 & 0.41790 & 1.03990 & $0.2130^{*}$ \\
\hline H10S & 0.73720 & 0.49490 & 1.08470 & $0.2130^{*}$ \\
\hline $\mathrm{H} 10 \mathrm{~T}$ & 0.79570 & 0.35070 & 1.09880 & $0.2980 *$ \\
\hline $\mathrm{H} 10 \mathrm{U}$ & 0.84120 & 0.42550 & 1.07190 & $0.2980 *$ \\
\hline $\mathrm{H} 10 \mathrm{~V}$ & 0.79800 & 0.35020 & 1.02420 & $0.2980 *$ \\
\hline H10W & 0.81600 & 0.56360 & 1.10630 & $0.3040^{*}$ \\
\hline H10Y & 0.83690 & 0.54920 & 1.00190 & $0.1430^{*}$ \\
\hline
\end{tabular}




$\begin{array}{lllll}\text { H10Z } & 0.78760 & 0.63550 & 0.97800 & 0.1430^{*} \\ \text { H2QW } & 0.77380 & 0.87880 & 1.08290 & 0.2600^{*} \\ \text { H20G } & 0.86200 & 1.00060 & 0.97140 & 0.0880^{*} \\ \text { H20H } & 0.84860 & 0.93320 & 1.02640 & 0.0880^{*} \\ \text { H20I } & 0.85960 & 0.84940 & 0.93800 & 0.2050^{*} \\ \text { H20J } & 0.79720 & 0.90520 & 0.89310 & 0.2050^{*} \\ \text { H20K } & 0.78260 & 0.83790 & 0.94810 & 0.2050^{*} \\ \text { H20L } & 0.69170 & 1.10150 & 0.93170 & 0.0960^{*} \\ \text { H20M } & 0.70850 & 1.01230 & 0.89420 & 0.0960^{*} \\ \text { H20N } & 0.75280 & 1.14580 & 0.85140 & 0.1990^{*} \\ \text { H20O } & 0.81660 & 1.08140 & 0.88420 & 0.1990^{*} \\ \text { H20P } & 0.79900 & 1.17110 & 0.92110 & 0.1990^{*} \\ \text { H20Q } & 0.69430 & 0.87640 & 1.08700 & 0.2600^{*} \\ \text { H20R } & 0.82480 & 1.12770 & 1.02540 & 0.1280^{*} \\ \text { H20S } & 0.81640 & 1.05560 & 1.07980 & 0.1280^{*} \\ \text { H20T } & 0.75410 & 1.19430 & 1.09160 & 0.2520^{*} \\ \text { H20U } & 0.70000 & 1.11130 & 1.07380 & 0.2520^{*} \\ \text { H20V } & 0.70870 & 1.18360 & 1.01950 & 0.2520^{*} \\ \text { H20W } & 0.73990 & 0.96660 & 1.10920 & 0.2600^{*} \\ \text { H20Y } & 0.70010 & 0.90150 & 0.97860 & 0.1230^{*} \\ \text { H20Z } & 0.66640 & 0.98910 & 1.00480 & 0.1230^{*}\end{array}$

Atomic displacement parameters $\left(\AA^{2}\right)$

\begin{tabular}{lllllll}
\hline & $U^{11}$ & $U^{22}$ & $U^{33}$ & $U^{12}$ & $U^{13}$ & $U^{23}$ \\
\hline S1 & $0.0502(7)$ & $0.0276(6)$ & $0.0421(6)$ & $-0.0061(6)$ & $0.0242(5)$ & $-0.0121(6)$ \\
S2 & $0.0481(7)$ & $0.0233(5)$ & $0.0469(7)$ & $-0.0079(6)$ & $0.0232(6)$ & $-0.0039(6)$ \\
S3 & $0.0391(6)$ & $0.0228(5)$ & $0.0477(7)$ & $0.0044(6)$ & $-0.0029(5)$ & $0.0039(6)$ \\
S4 & $0.0399(6)$ & $0.0267(5)$ & $0.0322(5)$ & $-0.0033(5)$ & $-0.0057(4)$ & $-0.0051(5)$ \\
S1' & $0.0349(6)$ & $0.0282(6)$ & $0.0407(6)$ & $-0.0020(6)$ & $-0.0024(5)$ & $-0.0047(5)$ \\
S2 $^{\prime}$ & $0.0340(6)$ & $0.0250(6)$ & $0.0509(7)$ & $0.0050(5)$ & $0.0035(5)$ & $0.0017(6)$ \\
S3' & $0.0425(6)$ & $0.0236(5)$ & $0.0490(7)$ & $-0.0033(6)$ & $0.0182(5)$ & $0.0043(6)$ \\
S4' & $0.0453(6)$ & $0.0283(6)$ & $0.0307(5)$ & $-0.0011(6)$ & $0.0160(5)$ & $-0.0082(5)$ \\
C11 & $0.269(8)$ & $0.315(11)$ & $0.201(6)$ & $0.065(9)$ & $-0.013(6)$ & $-0.056(7)$ \\
O1 & $0.046(2)$ & $0.0318(19)$ & $0.0434(19)$ & $-0.0051(18)$ & $0.0139(17)$ & $-0.0103(16)$ \\
O2 & $0.049(2)$ & $0.0238(16)$ & $0.0258(15)$ & $-0.0009(16)$ & $0.0066(14)$ & $0.0030(13)$ \\
O3 & $0.053(2)$ & $0.0269(17)$ & $0.0362(18)$ & $-0.0054(17)$ & $0.0062(16)$ & $-0.0052(15)$ \\
O4 & $0.066(2)$ & $0.0207(15)$ & $0.0177(13)$ & $-0.0040(17)$ & $0.0061(14)$ & $-0.0029(12)$ \\
O5 & $0.158(6)$ & $0.0225(19)$ & $0.0256(17)$ & $0.005(3)$ & $0.008(3)$ & $-0.0005(15)$ \\
C12 & $0.285(9)$ & $0.313(11)$ & $0.217(6)$ & $0.072(9)$ & $0.073(6)$ & $0.024(7)$ \\
C1 & $0.048(3)$ & $0.031(3)$ & $0.042(3)$ & $0.000(2)$ & $0.029(2)$ & $-0.003(2)$ \\
O1' & $0.040(2)$ & $0.0313(19)$ & $0.045(2)$ & $-0.0028(17)$ & $0.0109(16)$ & $-0.0065(16)$ \\
C2 & $0.045(3)$ & $0.029(2)$ & $0.036(2)$ & $-0.007(2)$ & $0.023(2)$ & $-0.008(2)$ \\
O2' & $0.0395(18)$ & $0.0229(16)$ & $0.0308(16)$ & $-0.0003(15)$ & $0.0046(14)$ & $0.0044(13)$ \\
C3 & $0.041(3)$ & $0.031(2)$ & $0.032(2)$ & $-0.010(2)$ & $0.023(2)$ & $-0.0080(19)$ \\
O3' & $0.045(2)$ & $0.0293(18)$ & $0.0340(17)$ & $0.0031(17)$ & $0.0033(15)$ & $-0.0058(15)$ \\
C4 & $0.043(3)$ & $0.028(2)$ & $0.036(2)$ & $-0.007(2)$ & $0.022(2)$ & $-0.005(2)$ \\
O4' & $0.052(2)$ & $0.0217(15)$ & $0.0186(13)$ & $-0.0006(15)$ & $0.0011(13)$ & $-0.0004(12)$ \\
& & & & & &
\end{tabular}




\begin{tabular}{|c|c|c|c|c|c|c|}
\hline C5 & $0.049(3)$ & $0.030(3)$ & $0.036(2)$ & $-0.008(2)$ & $0.020(2)$ & $-0.005(2)$ \\
\hline O5' & $0.155(6)$ & $0.026(2)$ & $0.0244(17)$ & $-0.008(3)$ & $0.000(2)$ & $-0.0017(16)$ \\
\hline C6 & $0.053(3)$ & $0.033(3)$ & $0.040(3)$ & $-0.008(3)$ & $0.024(2)$ & $-0.006(2)$ \\
\hline $\mathrm{C} 7$ & $0.054(3)$ & $0.040(3)$ & $0.045(3)$ & $-0.001(3)$ & $0.009(3)$ & $-0.005(3)$ \\
\hline $\mathrm{C} 8$ & $0.096(8)$ & $0.060(6)$ & $0.161(11)$ & $0.013(6)$ & $-0.051(8)$ & $-0.006(7)$ \\
\hline C9 & $0.049(4)$ & $0.103(7)$ & $0.074(5)$ & $-0.004(5)$ & $0.017(4)$ & $0.006(5)$ \\
\hline $\mathrm{C} 10$ & $0.086(6)$ & $0.162(12)$ & $0.062(5)$ & $-0.003(8)$ & $0.004(5)$ & $-0.043(7)$ \\
\hline $\mathrm{C} 11$ & $0.037(2)$ & $0.029(2)$ & $0.033(2)$ & $-0.002(2)$ & $0.0072(18)$ & $-0.0043(19)$ \\
\hline $\mathrm{C} 12$ & $0.037(2)$ & 0.0188 (19) & $0.031(2)$ & $-0.003(2)$ & $0.0117(17)$ & $-0.0015(18)$ \\
\hline $\mathrm{C} 13$ & $0.044(3)$ & $0.016(2)$ & $0.0256(19)$ & $-0.0004(18)$ & $0.0050(18)$ & $0.0030(15)$ \\
\hline $\mathrm{C} 14$ & $0.035(2)$ & $0.0163(18)$ & $0.034(2)$ & $0.0021(19)$ & $0.0032(17)$ & $0.0014(18)$ \\
\hline $\mathrm{C} 15$ & $0.040(3)$ & $0.022(2)$ & $0.035(2)$ & $0.001(2)$ & $0.0122(19)$ & $-0.0015(18)$ \\
\hline $\mathrm{C} 16$ & $0.040(2)$ & $0.028(2)$ & $0.026(2)$ & $0.003(2)$ & $0.0091(18)$ & $-0.0025(18)$ \\
\hline $\mathrm{C} 17$ & $0.049(3)$ & $0.040(3)$ & $0.030(2)$ & $0.005(3)$ & $0.006(2)$ & $-0.004(2)$ \\
\hline $\mathrm{C} 18$ & $0.085(6)$ & $0.146(10)$ & $0.030(3)$ & $0.046(6)$ & $-0.009(3)$ & $-0.018(4)$ \\
\hline $\mathrm{C} 19$ & $0.097(6)$ & $0.059(4)$ & $0.046(3)$ & $0.017(4)$ & $0.008(4)$ & $-0.020(3)$ \\
\hline $\mathrm{C} 20$ & $0.114(7)$ & $0.101(7)$ & 0.039 (3) & $-0.014(7)$ & $0.031(4)$ & $-0.001(4)$ \\
\hline $\mathrm{C} 21$ & 0.035 & $0.032(3)$ & $0.043(3)$ & $0.003(2)$ & $-0.002(2)$ & $-0.003(2)$ \\
\hline $\mathrm{C} 22$ & 0.038 & $0.025(2)$ & $0.034(2)$ & $0.003(2)$ & $-0.0076(19)$ & 0.0024 (19) \\
\hline $\mathrm{C} 23$ & $0.033(2)$ & $0.026(2)$ & 0.0250 (19) & $0.0000(19)$ & $-0.0063(16)$ & $-0.0010(17)$ \\
\hline $\mathrm{C} 24$ & $0.036(2)$ & $0.028(2)$ & $0.034(2)$ & $-0.002(2)$ & $-0.0070(19)$ & $-0.0021(19)$ \\
\hline $\mathrm{C} 25$ & 0.035 & $0.030(2)$ & $0.040(3)$ & $0.000(2)$ & $-0.007(2)$ & $0.000(2)$ \\
\hline $\mathrm{C} 26$ & $0.033(3)$ & $0.036(3)$ & $0.044(3)$ & $-0.002(2)$ & $-0.002(2)$ & $-0.002(2)$ \\
\hline $\mathrm{C} 27$ & $0.050(3)$ & $0.044(3)$ & $0.055(4)$ & $-0.001(3)$ & $0.016(3)$ & $-0.001(3)$ \\
\hline $\mathrm{C} 28$ & $0.064(5)$ & $0.112(8)$ & $0.056(4)$ & $0.007(5)$ & $0.015(4)$ & $0.005(5)$ \\
\hline $\mathrm{C} 29$ & $0.042(4)$ & $0.104(7)$ & $0.082(5)$ & $0.007(5)$ & $0.010(4)$ & $-0.003(5)$ \\
\hline C30 & $0.169(12)$ & $0.052(5)$ & $0.162(11)$ & $-0.030(7)$ & $0.122(10)$ & $-0.013(6)$ \\
\hline C31 & $0.032(2)$ & $0.026(2)$ & $0.0192(17)$ & $-0.0020(18)$ & $0.0052(15)$ & $-0.0003(16)$ \\
\hline $\mathrm{C} 32$ & $0.033(2)$ & $0.0168(18)$ & $0.0203(16)$ & $-0.0033(18)$ & $0.0005(14)$ & $0.0020(15)$ \\
\hline $\mathrm{C} 33$ & $0.049(3)$ & $0.0166(19)$ & $0.0166(16)$ & $-0.001(2)$ & $0.0056(16)$ & $-0.0005(15)$ \\
\hline C34 & $0.037(2)$ & $0.022(2)$ & $0.0267(18)$ & $-0.001(2)$ & $0.0123(16)$ & $-0.0022(17)$ \\
\hline C35 & $0.033(2)$ & $0.030(2)$ & 0.0246 (19) & $0.001(2)$ & $0.0030(16)$ & $-0.0044(17)$ \\
\hline C36 & $0.036(2)$ & $0.025(2)$ & $0.0196(17)$ & $-0.0012(19)$ & $0.0047(16)$ & $-0.0021(16)$ \\
\hline C37 & $0.037(2)$ & $0.045(3)$ & $0.0186(18)$ & $0.000(2)$ & $0.0036(17)$ & $-0.0048(19)$ \\
\hline C38 & $0.069(5)$ & $0.147(10)$ & $0.035(3)$ & $0.012(6)$ & $0.007(3)$ & $-0.036(5)$ \\
\hline C39 & $0.137(8)$ & $0.071(5)$ & $0.025(3)$ & $-0.002(6)$ & $0.011(4)$ & $0.007(3)$ \\
\hline $\mathrm{C} 40$ & $0.091(6)$ & $0.080(5)$ & $0.041(3)$ & $-0.040(5)$ & $0.019(4)$ & $-0.026(4)$ \\
\hline C41 & $0.063(3)$ & $0.019(2)$ & $0.0212(19)$ & $0.000(2)$ & $0.007(2)$ & $-0.0022(17)$ \\
\hline C42 & $0.060(3)$ & $0.023(2)$ & $0.0207(18)$ & $-0.002(2)$ & $0.0056(19)$ & $-0.0012(17)$ \\
\hline $\mathrm{C} 43$ & $0.047(3)$ & $0.021(2)$ & $0.026(2)$ & $0.007(2)$ & $0.0092(19)$ & 0.0029 (17) \\
\hline $\mathrm{C} 44$ & $0.061(3)$ & $0.026(2)$ & 0.0207 (19) & $0.001(2)$ & $0.009(2)$ & $-0.0033(18)$ \\
\hline $\mathrm{C} 45$ & $0.070(4)$ & $0.034(3)$ & $0.021(2)$ & $0.007(3)$ & $0.010(2)$ & $0.001(2)$ \\
\hline $\mathrm{C} 46$ & $0.065(4)$ & $0.033(3)$ & $0.032(2)$ & $0.010(3)$ & $0.011(2)$ & $0.013(2)$ \\
\hline $\mathrm{C} 47$ & $0.072(4)$ & $0.021(2)$ & $0.035(2)$ & $0.008(3)$ & $0.014(3)$ & $0.001(2)$ \\
\hline $\mathrm{C} 48$ & $0.056(3)$ & $0.027(2)$ & $0.028(2)$ & $0.001(2)$ & $0.013(2)$ & $-0.0021(19)$ \\
\hline $\mathrm{C} 1^{\prime}$ & $0.031(2)$ & $0.037(3)$ & $0.045(3)$ & $0.001(2)$ & $0.000(2)$ & $0.007(2)$ \\
\hline $\mathrm{C} 2^{\prime}$ & $0.031(2)$ & $0.032(3)$ & $0.036(2)$ & $0.001(2)$ & $-0.0024(19)$ & $-0.001(2)$ \\
\hline $\mathrm{C} 3^{\prime}$ & $0.024(2)$ & $0.032(2)$ & $0.033(2)$ & $0.0054(19)$ & $-0.0044(16)$ & $-0.0002(19)$ \\
\hline
\end{tabular}




\begin{tabular}{|c|c|c|c|c|c|c|}
\hline $\mathrm{C} 4^{\prime}$ & $0.029(2)$ & $0.032(2)$ & $0.038(2)$ & $0.004(2)$ & $-0.0013(18)$ & $0.001(2)$ \\
\hline $\mathrm{C} 5^{\prime}$ & $0.039(3)$ & $0.033(3)$ & $0.040(3)$ & $0.006(2)$ & $0.003(2)$ & $0.002(2)$ \\
\hline $\mathrm{C} 6^{\prime}$ & $0.039(3)$ & $0.038(3)$ & $0.041(3)$ & $0.005(2)$ & $0.005(2)$ & $0.004(2)$ \\
\hline$C 7^{\prime}$ & $0.052(4)$ & $0.046(3)$ & $0.061(4)$ & $0.002(3)$ & $0.021(3)$ & $0.006(3)$ \\
\hline $\mathrm{C} 8^{\prime}$ & $0.152(10)$ & $0.066(6)$ & $0.120(8)$ & $-0.014(7)$ & $0.100(8)$ & $-0.007(6)$ \\
\hline $\mathrm{C} 9^{\prime}$ & $0.047(4)$ & $0.107(7)$ & $0.070(5)$ & $0.017(5)$ & $0.021(3)$ & $0.009(5)$ \\
\hline $\mathrm{C} 10^{\prime}$ & $0.087(6)$ & $0.100(7)$ & $0.068(5)$ & $-0.008(6)$ & $0.037(5)$ & $-0.015(5)$ \\
\hline $\mathrm{C} 11^{\prime}$ & $0.043(3)$ & $0.032(3)$ & $0.034(2)$ & $0.004(2)$ & $0.009(2)$ & $-0.002(2)$ \\
\hline $\mathrm{C} 12^{\prime}$ & $0.033(2)$ & 0.0198 (19) & $0.039(2)$ & $0.003(2)$ & $0.0045(17)$ & $0.001(2)$ \\
\hline C13' & $0.037(2)$ & $0.016(2)$ & $0.032(2)$ & $0.0019(18)$ & $0.0066(18)$ & $0.0036(16)$ \\
\hline $\mathrm{C} 14^{\prime}$ & $0.032(2)$ & 0.0191 (19) & $0.039(2)$ & $0.000(2)$ & $0.0098(17)$ & -0.0002 (19) \\
\hline $\mathrm{C} 15^{\prime}$ & $0.035(2)$ & $0.025(2)$ & $0.039(2)$ & $0.001(2)$ & 0.0029 (19) & $-0.004(2)$ \\
\hline $\mathrm{C} 16^{\prime}$ & $0.043(3)$ & $0.030(2)$ & $0.035(2)$ & $0.004(2)$ & $0.004(2)$ & $-0.002(2)$ \\
\hline $\mathrm{C} 17^{\prime}$ & $0.061(4)$ & $0.049(3)$ & $0.033(2)$ & $0.003(3)$ & $0.001(2)$ & -0.009 \\
\hline C18' & $0.108(7)$ & $0.139(10)$ & $0.043(4)$ & $-0.016(8)$ & $0.020(4)$ & $-0.015(5)$ \\
\hline C19' & $0.29(2)$ & $0.080(7)$ & $0.055(5)$ & $-0.045(10)$ & $-0.004(8)$ & $-0.028(5)$ \\
\hline $\mathrm{C} 20^{\prime}$ & $0.103(7)$ & $0.171(13)$ & $0.056(5)$ & $0.055(9)$ & $-0.021(5)$ & $-0.019(7)$ \\
\hline $\mathrm{C} 21^{\prime}$ & $0.034(2)$ & $0.033(3)$ & $0.036(2)$ & $-0.003(2)$ & $0.0121(19)$ & $-0.004(2)$ \\
\hline $\mathrm{C} 22^{\prime}$ & $0.038(2)$ & $0.029(2)$ & $0.030(2)$ & $-0.001(2)$ & 0.0152 (19) & 0.0038 (19) \\
\hline C23' & $0.038(2)$ & $0.029(2)$ & $0.0202(17)$ & $0.001(2)$ & $0.0113(16)$ & $-0.0022(17)$ \\
\hline C24' & $0.048(3)$ & $0.027(2)$ & $0.026(2)$ & $-0.001(2)$ & $0.018(2)$ & $-0.0054(18)$ \\
\hline $\mathrm{C} 25^{\prime}$ & $0.045(3)$ & $0.028(2)$ & $0.031(2)$ & $0.001(2)$ & $0.018(2)$ & $-0.0020(19)$ \\
\hline C26' & $0.040(3)$ & $0.035(3)$ & $0.034(2)$ & $0.001(2)$ & $0.015(2)$ & $-0.003(2)$ \\
\hline $\mathrm{C} 27^{\prime}$ & $0.041(3)$ & $0.043(3)$ & $0.039(3)$ & $0.003(3)$ & $0.011(2)$ & $-0.008(2)$ \\
\hline $\mathrm{C} 28^{\prime}$ & $0.059(4)$ & $0.069(5)$ & $0.039(3)$ & $0.001(4)$ & $0.004(3)$ & -0.005 \\
\hline C29' & $0.046(4)$ & $0.085(6)$ & $0.060(4)$ & $-0.003(4)$ & $0.019(3)$ & $0.000(4)$ \\
\hline $\mathrm{C} 30^{\prime}$ & $0.069(5)$ & $0.049(4)$ & $0.070(5)$ & $0.020(4)$ & $-0.011(4)$ & $-0.007(4)$ \\
\hline C31' & $0.030(2)$ & $0.029(2)$ & $0.0218(17)$ & 0.0049 (18) & $0.0041(15)$ & $-0.0021(16)$ \\
\hline C32' & $0.036(2)$ & 0.0187 (18) & $0.0228(17)$ & 0.0021 (19) & $0.0094(15)$ & $-0.0025(16)$ \\
\hline C33' & $0.040(2)$ & 0.0149 (18) & $0.0179(16)$ & $0.0010(18)$ & $0.0031(15)$ & $0.0005(15)$ \\
\hline C34' & $0.030(2)$ & $0.023(2)$ & 0.0277 (19) & $-0.003(2)$ & $0.0015(15)$ & $0.0018(18)$ \\
\hline C35' & $0.034(2)$ & $0.033(2)$ & 0.0255 (19) & $-0.001(2)$ & 0.0068 (17) & 0.0017 (18) \\
\hline C $36^{\prime}$ & $0.036(2)$ & $0.030(2)$ & $0.0212(18)$ & $0.004(2)$ & 0.0057 (17) & $0.0015(17)$ \\
\hline C37' & $0.043(3)$ & $0.047(3)$ & 0.0205 (19) & $0.001(3)$ & $0.0068(18)$ & $-0.003(2)$ \\
\hline C $38^{\prime}$ & $0.067(5)$ & $0.156(11)$ & $0.040(3)$ & $-0.018(6)$ & $0.004(3)$ & $-0.038(5)$ \\
\hline C39' & $0.201(13)$ & $0.083(7)$ & $0.026(3)$ & $-0.013(8)$ & $0.012(5)$ & $0.008(4)$ \\
\hline $\mathrm{C} 40^{\prime}$ & $0.079(6)$ & $0.138(9)$ & $0.066(5)$ & $0.040(6)$ & $-0.007(4)$ & $-0.066(6)$ \\
\hline C41' & $0.052(3)$ & $0.019(2)$ & $0.0211(18)$ & $-0.002(2)$ & $0.0021(18)$ & $0.0002(16)$ \\
\hline C42' & $0.053(3)$ & $0.024(2)$ & 0.0237 (19) & $-0.002(2)$ & 0.0040 (19) & $-0.0012(17)$ \\
\hline C43' & $0.044(3)$ & $0.024(2)$ & 0.0228 (19) & $-0.006(2)$ & $0.0019(18)$ & $0.0019(17)$ \\
\hline C44' & $0.059(3)$ & $0.028(2)$ & 0.0201 (19) & $-0.001(2)$ & $0.000(2)$ & $-0.0028(18)$ \\
\hline C45' & $0.072(4)$ & $0.036(3)$ & $0.020(2)$ & $-0.005(3)$ & $0.003(2)$ & $0.001(2)$ \\
\hline C46' & $0.064(4)$ & $0.030(3)$ & $0.032(2)$ & $-0.004(3)$ & $0.007(2)$ & $0.010(2)$ \\
\hline C47' & $0.065(4)$ & $0.024(2)$ & $0.036(3)$ & $-0.002(3)$ & $0.005(2)$ & $0.003(2)$ \\
\hline C48' & $0.050(3)$ & $0.027(2)$ & $0.027(2)$ & $-0.005(2)$ & $0.003(2)$ & -0.0018 (19) \\
\hline N1 & $0.051(3)$ & $0.059(3)$ & $0.047(3)$ & $0.001(3)$ & $0.028(2)$ & $0.017(3)$ \\
\hline C101 & $0.072(6)$ & $0.092(7)$ & $0.112(8)$ & $0.020(6)$ & $0.028(5)$ & $0.040(6)$ \\
\hline C102 & $0.119(10)$ & $0.093(9)$ & 0.248 (19) & $0.028(8)$ & $0.076(12)$ & $0.085(11)$ \\
\hline
\end{tabular}




\begin{tabular}{lllllll} 
& & & & \\
C103 & $0.123(9)$ & $0.105(8)$ & $0.081(6)$ & $0.043(7)$ & $0.055(6)$ & $0.039(6)$ \\
C104 & $0.158(13)$ & $0.199(17)$ & $0.094(8)$ & $-0.106(13)$ & $0.042(8)$ & $-0.058(10)$ \\
C105 & $0.171(12)$ & $0.29(2)$ & $0.114(9)$ & $0.168(14)$ & $0.114(9)$ & $0.145(12)$ \\
C106 & $0.202(17)$ & $0.31(3)$ & $0.110(10)$ & $0.180(19)$ & $0.083(11)$ & $0.110(14)$ \\
C107 & $0.097(8)$ & $0.145(12)$ & $0.126(9)$ & $0.001(9)$ & $0.047(7)$ & $-0.010(9)$ \\
C108 & $0.129(12)$ & $0.33(3)$ & $0.164(13)$ & $-0.081(16)$ & $0.067(11)$ & $-0.150(18)$ \\
N2 & $0.034(2)$ & $0.058(3)$ & $0.046(3)$ & $-0.004(2)$ & $-0.0045(19)$ & $-0.017(2)$ \\
C201 & $0.053(4)$ & $0.076(5)$ & $0.086(5)$ & $0.006(4)$ & $0.006(4)$ & $-0.035(5)$ \\
C202 & $0.089(8)$ & $0.090(8)$ & $0.231(16)$ & $0.011(7)$ & $0.035(9)$ & $-0.077(10)$ \\
C203 & $0.068(5)$ & $0.102(7)$ & $0.060(4)$ & $0.010(5)$ & $-0.005(4)$ & $-0.024(5)$ \\
C204 & $0.125(10)$ & $0.161(13)$ & $0.097(8)$ & $-0.059(10)$ & $-0.006(7)$ & $0.051(9)$ \\
C205 & $0.079(6)$ & $0.139(10)$ & $0.081(6)$ & $0.038(6)$ & $-0.028(5)$ & $-0.077(7)$ \\
C206 & $0.113(9)$ & $0.219(18)$ & $0.140(11)$ & $0.091(11)$ & $-0.039(8)$ & $-0.127(12)$ \\
C207 & $0.069(6)$ & $0.140(11)$ & $0.102(8)$ & $-0.016(7)$ & $0.026(5)$ & $-0.025(8)$ \\
C208 & $0.089(8)$ & $0.29(2)$ & $0.141(12)$ & $0.002(13)$ & $0.028(8)$ & $0.129(15)$ \\
& & & & & \\
\hline
\end{tabular}

Geometric parameters $\left(A,{ }^{\circ}\right)$

\begin{tabular}{|c|c|c|c|}
\hline $\mathrm{S} 1-\mathrm{C} 2$ & $1.773(6)$ & $\mathrm{C} 11^{\prime}-\mathrm{C} 16^{\prime}$ & $1.404(9)$ \\
\hline $\mathrm{S} 1-\mathrm{C} 34$ & $1.781(6)$ & $\mathrm{C} 12^{\prime}-\mathrm{C} 13^{\prime}$ & $1.424(8)$ \\
\hline $\mathrm{S} 2-\mathrm{C} 4$ & $1.785(6)$ & $\mathrm{C} 13^{\prime}-\mathrm{C} 14^{\prime}$ & $1.419(7)$ \\
\hline $\mathrm{S} 2-\mathrm{C} 12$ & $1.777(4)$ & $\mathrm{C} 14^{\prime}-\mathrm{C} 15^{\prime}$ & $1.387(7)$ \\
\hline $\mathrm{S} 3-\mathrm{C} 14$ & $1.776(4)$ & $\mathrm{C} 15^{\prime}-\mathrm{C} 16^{\prime}$ & $1.391(9)$ \\
\hline $\mathrm{S} 3-\mathrm{C} 22$ & $1.784(6)$ & $\mathrm{C} 16^{\prime}-\mathrm{C} 17^{\prime}$ & $1.529(9)$ \\
\hline $\mathrm{S} 4-\mathrm{C} 24$ & $1.784(6)$ & $\mathrm{C} 17^{\prime}-\mathrm{C} 20^{\prime}$ & $1.548(15)$ \\
\hline $\mathrm{S} 4-\mathrm{C} 32$ & $1.780(4)$ & $\mathrm{C} 17^{\prime}-\mathrm{C} 19^{\prime}$ & $1.490(14)$ \\
\hline $\mathrm{S} 1^{\prime}-\mathrm{C} 34^{\prime}$ & $1.782(4)$ & $\mathrm{C} 17^{\prime}-\mathrm{C} 18^{\prime}$ & $1.493(13)$ \\
\hline $\mathrm{S} 1^{\prime}-\mathrm{C} 2^{\prime}$ & $1.766(6)$ & $\mathrm{C} 21^{\prime}-\mathrm{C} 22^{\prime}$ & $1.394(8)$ \\
\hline $\mathrm{S} 2^{\prime}-\mathrm{C} 12^{\prime}$ & $1.776(6)$ & $\mathrm{C} 21^{\prime}-\mathrm{C} 26^{\prime}$ & $1.397(8)$ \\
\hline $\mathrm{S} 2^{\prime}-\mathrm{C} 4^{\prime}$ & $1.776(6)$ & $\mathrm{C} 22^{\prime}-\mathrm{C} 23^{\prime}$ & $1.400(8)$ \\
\hline $\mathrm{S} 3^{\prime}-\mathrm{C} 14^{\prime}$ & $1.776(4)$ & $\mathrm{C} 23^{\prime}-\mathrm{C} 24^{\prime}$ & $1.421(8)$ \\
\hline $\mathrm{S} 3^{\prime}-\mathrm{C} 22^{\prime}$ & $1.771(6)$ & $\mathrm{C} 24^{\prime}-\mathrm{C} 25^{\prime}$ & $1.386(8)$ \\
\hline $\mathrm{S} 4^{\prime}-\mathrm{C} 24^{\prime}$ & $1.778(6)$ & $\mathrm{C} 25^{\prime}-\mathrm{C} 26^{\prime}$ & $1.407(8)$ \\
\hline $\mathrm{S} 4^{\prime}-\mathrm{C} 32^{\prime}$ & $1.782(4)$ & $\mathrm{C} 26^{\prime}-\mathrm{C} 27^{\prime}$ & $1.518(9)$ \\
\hline $\mathrm{O} 1-\mathrm{C} 3$ & $1.332(7)$ & $\mathrm{C} 27^{\prime}-\mathrm{C} 30^{\prime}$ & $1.543(10)$ \\
\hline $\mathrm{O} 2-\mathrm{C} 13$ & $1.304(5)$ & $\mathrm{C} 27^{\prime}-\mathrm{C} 28^{\prime}$ & $1.515(9)$ \\
\hline $\mathrm{O} 3-\mathrm{C} 23$ & $1.332(7)$ & $\mathrm{C} 27^{\prime}-\mathrm{C} 29^{\prime}$ & $1.540(10)$ \\
\hline $\mathrm{O} 4-\mathrm{C} 33$ & $1.380(5)$ & $\mathrm{C} 31^{\prime}-\mathrm{C} 32^{\prime}$ & $1.394(6)$ \\
\hline $\mathrm{O} 4-\mathrm{C} 41$ & $1.438(5)$ & $\mathrm{C} 31^{\prime}-\mathrm{C} 36^{\prime}$ & $1.398(8)$ \\
\hline $\mathrm{O} 5-\mathrm{C} 42$ & $1.205(7)$ & $\mathrm{C} 32^{\prime}-\mathrm{C} 33^{\prime}$ & $1.385(6)$ \\
\hline $\mathrm{O} 1-\mathrm{H} 1 \mathrm{O}$ & 0.8200 & C33'-C34' & $1.401(6)$ \\
\hline $\mathrm{O} 2-\mathrm{H} 2 \mathrm{O}$ & 0.8200 & $\mathrm{C} 34^{\prime}-\mathrm{C} 35^{\prime}$ & $1.385(6)$ \\
\hline $\mathrm{O} 3-\mathrm{H} 3 \mathrm{O}$ & 0.8200 & $\mathrm{C} 35^{\prime}-\mathrm{C} 36^{\prime}$ & $1.397(8)$ \\
\hline $\mathrm{C} 1-\mathrm{C} 6$ & $1.404(8)$ & $\mathrm{C} 36^{\prime}-\mathrm{C} 37^{\prime}$ & $1.532(6)$ \\
\hline $\mathrm{C} 1-\mathrm{C} 2$ & $1.388(9)$ & $\mathrm{C} 37^{\prime}-\mathrm{C} 38^{\prime}$ & $1.491(12)$ \\
\hline $\mathrm{O} 1^{\prime}-\mathrm{C} 3^{\prime}$ & $1.337(7)$ & $\mathrm{C} 37^{\prime}-\mathrm{C} 39^{\prime}$ & $1.496(11)$ \\
\hline $\mathrm{C} 2-\mathrm{C} 3$ & $1.400(8)$ & $\mathrm{C} 37^{\prime}-\mathrm{C} 40^{\prime}$ & $1.540(13)$ \\
\hline $\mathrm{O} 2^{\prime}-\mathrm{C} 13^{\prime}$ & $1.312(5)$ & $\mathrm{C} 41^{\prime}-\mathrm{C} 42^{\prime}$ & $1.496(6)$ \\
\hline
\end{tabular}




\begin{tabular}{|c|c|c|c|}
\hline $\mathrm{C} 3-\mathrm{C} 4$ & $1.412(8)$ & $\mathrm{C} 42^{\prime}-\mathrm{C} 43^{\prime}$ & $1.481(7)$ \\
\hline $\mathrm{O} 3^{\prime}-\mathrm{C} 23^{\prime}$ & $1.331(7)$ & $\mathrm{C} 43^{\prime}-\mathrm{C} 48^{\prime}$ & $1.373(8)$ \\
\hline $\mathrm{C} 4-\mathrm{C} 5$ & $1.384(8)$ & $\mathrm{C} 43^{\prime}-\mathrm{C} 44^{\prime}$ & $1.409(6)$ \\
\hline $\mathrm{O} 4^{\prime}-\mathrm{C} 33^{\prime}$ & $1.384(5)$ & $\mathrm{C} 44^{\prime}-\mathrm{C} 45^{\prime}$ & $1.370(7)$ \\
\hline $\mathrm{O} 4^{\prime}-\mathrm{C} 41^{\prime}$ & $1.430(5)$ & $\mathrm{C} 45^{\prime}-\mathrm{C} 46^{\prime}$ & $1.398(8)$ \\
\hline $\mathrm{C} 5-\mathrm{C} 6$ & $1.388(8)$ & $\mathrm{C} 46^{\prime}-\mathrm{C} 47^{\prime}$ & $1.373(9)$ \\
\hline $\mathrm{O} 5^{\prime}-\mathrm{C} 42^{\prime}$ & $1.209(7)$ & $\mathrm{C} 47^{\prime}-\mathrm{C} 48^{\prime}$ & $1.385(8)$ \\
\hline $\mathrm{C} 6-\mathrm{C} 7$ & $1.541(10)$ & $\mathrm{N} 1-\mathrm{C} 105$ & $1.479(17)$ \\
\hline $\mathrm{C} 7-\mathrm{C} 10$ & $1.530(13)$ & $\mathrm{N} 1-\mathrm{C} 107$ & $1.525(17)$ \\
\hline $\mathrm{C} 7-\mathrm{C} 9$ & $1.518(12)$ & $\mathrm{N} 1-\mathrm{C} 101$ & $1.526(12)$ \\
\hline $\mathrm{C} 7-\mathrm{C} 8$ & $1.504(14)$ & $\mathrm{N} 1-\mathrm{C} 103$ & $1.459(13)$ \\
\hline $\mathrm{C} 11-\mathrm{C} 12$ & $1.404(6)$ & $\mathrm{C} 1^{\prime}-\mathrm{H} 1^{\prime}$ & 0.9300 \\
\hline $\mathrm{C} 11-\mathrm{C} 16$ & $1.391(8)$ & $\mathrm{C} 5^{\prime}-\mathrm{H} 5^{\prime}$ & 0.9300 \\
\hline $\mathrm{C} 12-\mathrm{C} 13$ & $1.428(7)$ & $\mathrm{C} 8^{\prime}-\mathrm{H} 8 \mathrm{~B}^{\prime}$ & 0.9600 \\
\hline $\mathrm{C} 13-\mathrm{C} 14$ & $1.411(7)$ & $\mathrm{C} 8^{\prime}-\mathrm{H} 8 \mathrm{C}^{\prime}$ & 0.9600 \\
\hline $\mathrm{C} 14-\mathrm{C} 15$ & $1.401(6)$ & $\mathrm{C} 8^{\prime}-\mathrm{H} 8 \mathrm{~A}^{\prime}$ & 0.9600 \\
\hline $\mathrm{C} 15-\mathrm{C} 16$ & $1.392(8)$ & $\mathrm{C} 9^{\prime}-\mathrm{H} 9 \mathrm{C}^{\prime}$ & 0.9600 \\
\hline $\mathrm{C} 16-\mathrm{C} 17$ & $1.531(6)$ & $\mathrm{C} 9^{\prime}-\mathrm{H} 9 \mathrm{~A}^{\prime}$ & 0.9600 \\
\hline $\mathrm{C} 17-\mathrm{C} 20$ & $1.549(11)$ & $\mathrm{C} 9^{\prime}-\mathrm{H} 9 \mathrm{~B}^{\prime}$ & 0.9600 \\
\hline $\mathrm{C} 17-\mathrm{C} 18$ & $1.505(11)$ & $\mathrm{C} 10^{\prime}-\mathrm{H} 10 \mathrm{E}$ & 0.9600 \\
\hline $\mathrm{C} 17-\mathrm{C} 19$ & $1.516(10)$ & $\mathrm{C} 10^{\prime}-\mathrm{H} 10 \mathrm{~F}$ & 0.9600 \\
\hline $\mathrm{C} 21-\mathrm{C} 22$ & $1.377(8)$ & $\mathrm{C} 10^{\prime}-\mathrm{H} 10 \mathrm{D}$ & 0.9600 \\
\hline $\mathrm{C} 21-\mathrm{C} 26$ & $1.410(8)$ & $\mathrm{C} 11^{\prime}-\mathrm{H} 11^{\prime}$ & 0.9300 \\
\hline $\mathrm{C} 22-\mathrm{C} 23$ & $1.408(8)$ & $\mathrm{C} 15^{\prime}-\mathrm{H} 15^{\prime}$ & 0.9300 \\
\hline $\mathrm{C} 23-\mathrm{C} 24$ & $1.412(8)$ & $\mathrm{C} 18^{\prime}-\mathrm{H} 18 \mathrm{D}$ & 0.9600 \\
\hline $\mathrm{C} 24-\mathrm{C} 25$ & $1.391(8)$ & $\mathrm{C} 18^{\prime}-\mathrm{H} 18 \mathrm{E}$ & 0.9600 \\
\hline $\mathrm{C} 25-\mathrm{C} 26$ & $1.389(8)$ & $\mathrm{C} 18^{\prime}-\mathrm{H} 18 \mathrm{~F}$ & 0.9600 \\
\hline $\mathrm{C} 26-\mathrm{C} 27$ & $1.516(9)$ & C19'-H19E & 0.9600 \\
\hline $\mathrm{C} 27-\mathrm{C} 28$ & $1.531(11)$ & $\mathrm{C} 19^{\prime}-\mathrm{H} 19 \mathrm{~F}$ & 0.9600 \\
\hline $\mathrm{C} 27-\mathrm{C} 30$ & $1.527(13)$ & C19'-H19D & 0.9600 \\
\hline $\mathrm{C} 27-\mathrm{C} 29$ & $1.505(12)$ & $\mathrm{C} 20^{\prime}-\mathrm{H} 20 \mathrm{D}$ & 0.9600 \\
\hline $\mathrm{C} 31-\mathrm{C} 36$ & $1.383(7)$ & $\mathrm{C} 20^{\prime}-\mathrm{H} 20 \mathrm{~F}$ & 0.9600 \\
\hline $\mathrm{C} 31-\mathrm{C} 32$ & $1.393(6)$ & $\mathrm{C} 20^{\prime}-\mathrm{H} 20 \mathrm{E}$ & 0.9600 \\
\hline $\mathrm{C} 32-\mathrm{C} 33$ & $1.392(7)$ & $\mathrm{C} 21^{\prime}-\mathrm{H} 21^{\prime}$ & 0.9300 \\
\hline $\mathrm{C} 33-\mathrm{C} 34$ & $1.385(8)$ & $\mathrm{C} 25^{\prime}-\mathrm{H} 25^{\prime}$ & 0.9300 \\
\hline $\mathrm{C} 34-\mathrm{C} 35$ & $1.395(6)$ & $\mathrm{C} 28^{\prime}-\mathrm{H} 28 \mathrm{E}$ & 0.9600 \\
\hline $\mathrm{C} 35-\mathrm{C} 36$ & $1.403(8)$ & $\mathrm{C} 28^{\prime}-\mathrm{H} 28 \mathrm{~F}$ & 0.9600 \\
\hline $\mathrm{C} 36-\mathrm{C} 37$ & $1.543(6)$ & $\mathrm{C} 28^{\prime}-\mathrm{H} 28 \mathrm{D}$ & 0.9600 \\
\hline $\mathrm{C} 37-\mathrm{C} 38$ & $1.505(10)$ & $\mathrm{C} 29^{\prime}-\mathrm{H} 29 \mathrm{D}$ & 0.9600 \\
\hline $\mathrm{C} 37-\mathrm{C} 40$ & $1.535(11)$ & $\mathrm{C} 29^{\prime}-\mathrm{H} 29 \mathrm{E}$ & 0.9600 \\
\hline C37-C39 & $1.507(10)$ & $\mathrm{C} 29^{\prime}-\mathrm{H} 29 \mathrm{~F}$ & 0.9600 \\
\hline $\mathrm{C} 41-\mathrm{C} 42$ & $1.495(6)$ & $\mathrm{C} 30^{\prime}-\mathrm{H} 30 \mathrm{D}$ & 0.9600 \\
\hline $\mathrm{C} 42-\mathrm{C} 43$ & $1.482(6)$ & $\mathrm{C} 30^{\prime}-\mathrm{H} 30 \mathrm{E}$ & 0.9600 \\
\hline $\mathrm{C} 43-\mathrm{C} 48$ & $1.385(7)$ & $\mathrm{C} 30^{\prime}-\mathrm{H} 30 \mathrm{~F}$ & 0.9600 \\
\hline $\mathrm{C} 43-\mathrm{C} 44$ & $1.407(6)$ & $\mathrm{C} 31^{\prime}-\mathrm{H} 31^{\prime}$ & 0.9300 \\
\hline $\mathrm{C} 44-\mathrm{C} 45$ & $1.366(7)$ & $\mathrm{C} 35^{\prime}-\mathrm{H} 35^{\prime}$ & 0.9300 \\
\hline $\mathrm{C} 45-\mathrm{C} 46$ & $1.388(8)$ & $\mathrm{C} 38^{\prime}-\mathrm{H} 38 \mathrm{D}$ & 0.9600 \\
\hline $\mathrm{C} 46-\mathrm{C} 47$ & $1.378(9)$ & C $38^{\prime}-\mathrm{H} 38 \mathrm{E}$ & 0.9600 \\
\hline
\end{tabular}




\begin{tabular}{|c|c|c|c|}
\hline $\mathrm{C} 47-\mathrm{C} 48$ & $1.383(8)$ & $\mathrm{C} 38^{\prime}-\mathrm{H} 38 \mathrm{~F}$ & 0.9600 \\
\hline $\mathrm{C} 1-\mathrm{H} 1$ & 0.9300 & $\mathrm{C} 39^{\prime}-\mathrm{H} 39 \mathrm{~F}$ & 0.9600 \\
\hline $\mathrm{O} 1^{\prime}-\mathrm{H} 6 \mathrm{O}$ & 0.8200 & C39'-H39D & 0.9600 \\
\hline $\mathrm{O} 2^{\prime}-\mathrm{H} 7 \mathrm{O}$ & 0.8200 & C39'-H39E & 0.9600 \\
\hline $\mathrm{O}^{\prime}-\mathrm{H} 8 \mathrm{O}$ & 0.8200 & $\mathrm{C} 40^{\prime}-\mathrm{H} 40 \mathrm{E}$ & 0.9600 \\
\hline $\mathrm{C} 5-\mathrm{H} 5$ & 0.9300 & $\mathrm{C} 40^{\prime}-\mathrm{H} 40 \mathrm{~F}$ & 0.9600 \\
\hline $\mathrm{C} 8-\mathrm{H} 8 \mathrm{~A}$ & 0.9600 & $\mathrm{C} 40^{\prime}-\mathrm{H} 40 \mathrm{D}$ & 0.9600 \\
\hline $\mathrm{C} 8-\mathrm{H} 8 \mathrm{~B}$ & 0.9600 & $\mathrm{C} 41^{\prime}-\mathrm{H} 41 \mathrm{C}$ & 0.9700 \\
\hline $\mathrm{C} 8-\mathrm{H} 8 \mathrm{C}$ & 0.9600 & $\mathrm{C} 41^{\prime}-\mathrm{H} 41 \mathrm{D}$ & 0.9700 \\
\hline С9- & 0.9600 & $\mathrm{C} 44^{\prime}-\mathrm{H} 44^{\prime}$ & 0.9300 \\
\hline C9- $\mathrm{H} 9 \mathrm{~A}$ & 0.9600 & $\mathrm{C} 45^{\prime}-\mathrm{H} 45^{\prime}$ & 0.9300 \\
\hline C9-H9C & 0.9600 & $\mathrm{C} 46^{\prime}-\mathrm{H} 46^{\prime}$ & 0.9300 \\
\hline $\mathrm{C} 10-\mathrm{H} 10 \mathrm{C}$ & 0.9600 & $\mathrm{C} 47^{\prime}-\mathrm{H} 47^{\prime}$ & 0.9300 \\
\hline $\mathrm{C} 10-\mathrm{H} 10 \mathrm{~B}$ & 0.9600 & $\mathrm{C} 48^{\prime}-\mathrm{H} 48^{\prime}$ & 0.9300 \\
\hline $\mathrm{C} 10-\mathrm{H} 10 \mathrm{~A}$ & 0.9600 & $\mathrm{C} 101-\mathrm{C} 102$ & $1.42(2)$ \\
\hline C11-H11 & 0.9300 & $\mathrm{C} 103-\mathrm{C} 104$ & $1.57(2)$ \\
\hline C15-H15 & 0.9300 & $\mathrm{C} 105-\mathrm{C} 106$ & $1.51(3)$ \\
\hline C18-H18B & 0.9600 & $\mathrm{C} 107-\mathrm{C} 108$ & $1.49(2)$ \\
\hline $\mathrm{C} 18-\mathrm{H} 18 \mathrm{~A}$ & 0.9600 & $\mathrm{~N} 2-\mathrm{C} 205$ & $1.496(13)$ \\
\hline $\mathrm{C} 18-\mathrm{H} 18 \mathrm{C}$ & 0.9600 & $\mathrm{~N} 2-\mathrm{C} 207$ & $1.493(15)$ \\
\hline C19-H19B & 0.9600 & $\mathrm{~N} 2-\mathrm{C} 201$ & $1.525(11)$ \\
\hline $\mathrm{C} 19-\mathrm{H} 19 \mathrm{C}$ & 0.9600 & $\mathrm{~N} 2-\mathrm{C} 203$ & $1.482(11)$ \\
\hline C19-H19A & 0.9600 & $\mathrm{C} 101-\mathrm{H} 10 \mathrm{G}$ & 0.9700 \\
\hline $\mathrm{C} 20-\mathrm{H} 20 \mathrm{~B}$ & 0.9600 & $\mathrm{C} 101-\mathrm{H} 10 \mathrm{H}$ & 0.9700 \\
\hline $\mathrm{C} 20-\mathrm{H} 20 \mathrm{~A}$ & 0.9600 & C102-H10J & 0.9600 \\
\hline $\mathrm{C} 20-\mathrm{H} 20 \mathrm{C}$ & 0.9600 & $\mathrm{C} 102-\mathrm{H} 10 \mathrm{~K}$ & 0.9600 \\
\hline $\mathrm{C} 21-\mathrm{H} 21$ & 0.9300 & C102-H10I & 0.9600 \\
\hline $\mathrm{C} 25-\mathrm{H} 25$ & 0.9300 & $\mathrm{C} 103-\mathrm{H} 10 \mathrm{~L}$ & 0.9700 \\
\hline $\mathrm{C} 28-\mathrm{H} 28 \mathrm{~A}$ & 0.9600 & $\mathrm{C} 103-\mathrm{H} 10 \mathrm{M}$ & 0.9700 \\
\hline $\mathrm{C} 28-\mathrm{H} 28 \mathrm{C}$ & 0.9600 & $\mathrm{C} 104-\mathrm{H} 10 \mathrm{P}$ & 0.9600 \\
\hline $\mathrm{C} 28-\mathrm{H} 28 \mathrm{~B}$ & 0.9600 & $\mathrm{C} 104-\mathrm{H} 10 \mathrm{~N}$ & 0.9600 \\
\hline $\mathrm{C} 29-\mathrm{H} 29 \mathrm{~B}$ & 0.9600 & $\mathrm{C} 104-\mathrm{H} 10 \mathrm{O}$ & 0.9600 \\
\hline $\mathrm{C} 29-\mathrm{H} 29 \mathrm{C}$ & 0.9600 & C105-H10S & 0.9700 \\
\hline $\mathrm{C} 29-\mathrm{H} 29 \mathrm{~A}$ & 0.9600 & $\mathrm{C} 105-\mathrm{H} 10 \mathrm{R}$ & 0.9700 \\
\hline $\mathrm{C} 30-\mathrm{H} 30 \mathrm{C}$ & 0.9600 & $\mathrm{C} 106-\mathrm{H} 10 \mathrm{~T}$ & 0.9600 \\
\hline $\mathrm{C} 30-\mathrm{H} 30 \mathrm{~B}$ & 0.9600 & $\mathrm{C} 106-\mathrm{H} 10 \mathrm{U}$ & 0.9600 \\
\hline $\mathrm{C} 30-\mathrm{H} 30 \mathrm{~A}$ & 0.9600 & $\mathrm{C} 106-\mathrm{H} 10 \mathrm{~V}$ & 0.9600 \\
\hline C31-H31 & 0.9300 & $\mathrm{C} 107-\mathrm{H} 10 \mathrm{Y}$ & 0.9700 \\
\hline $\mathrm{C} 35-\mathrm{H} 35$ & 0.9300 & $\mathrm{C} 107-\mathrm{H} 10 \mathrm{Z}$ & 0.9700 \\
\hline $\mathrm{C} 38-\mathrm{H} 38 \mathrm{C}$ & 0.9600 & $\mathrm{C} 108-\mathrm{H} 10 \mathrm{Q}$ & 0.9600 \\
\hline C38-H38A & 0.9600 & C108-H10W & 0.9600 \\
\hline $\mathrm{C} 38-\mathrm{H} 38 \mathrm{~B}$ & 0.9600 & C108-H1QW & 0.9600 \\
\hline $\mathrm{C} 39-\mathrm{H} 39 \mathrm{C}$ & 0.9600 & $\mathrm{C} 201-\mathrm{C} 202$ & $1.449(18)$ \\
\hline C39-H39B & 0.9600 & C203-C204 & $1.548(19)$ \\
\hline C39-H39A & 0.9600 & C205-C206 & $1.55(2)$ \\
\hline $\mathrm{C} 40-\mathrm{H} 40 \mathrm{~B}$ & 0.9600 & C207-C208 & $1.52(2)$ \\
\hline $\mathrm{C} 40-\mathrm{H} 40 \mathrm{~A}$ & 0.9600 & $\mathrm{C} 201-\mathrm{H} 20 \mathrm{G}$ & 0.9700 \\
\hline $\mathrm{C} 40-\mathrm{H} 40 \mathrm{C}$ & 0.9600 & $\mathrm{C} 201-\mathrm{H} 20 \mathrm{H}$ & 0.9700 \\
\hline
\end{tabular}




\begin{tabular}{|c|c|c|c|}
\hline $\mathrm{C} 41-\mathrm{H} 41 \mathrm{~B}$ & 0.9700 & $\mathrm{C} 202-\mathrm{H} 20 \mathrm{I}$ & 0.9600 \\
\hline $\mathrm{C} 41-\mathrm{H} 41 \mathrm{~A}$ & 0.9700 & $\mathrm{C} 202-\mathrm{H} 20 \mathrm{~J}$ & 0.9600 \\
\hline $\mathrm{C} 44-\mathrm{H} 44$ & 0.9300 & $\mathrm{C} 202-\mathrm{H} 20 \mathrm{~K}$ & 0.9600 \\
\hline $\mathrm{C} 45-\mathrm{H} 45$ & 0.9300 & $\mathrm{C} 203-\mathrm{H} 20 \mathrm{~L}$ & 0.9700 \\
\hline $\mathrm{C} 46-\mathrm{H} 46$ & 0.9300 & $\mathrm{C} 203-\mathrm{H} 20 \mathrm{M}$ & 0.9700 \\
\hline $\mathrm{C} 47-\mathrm{H} 47$ & 0.9300 & $\mathrm{C} 204-\mathrm{H} 20 \mathrm{~N}$ & 0.9600 \\
\hline $\mathrm{C} 48-\mathrm{H} 48$ & 0.9300 & $\mathrm{C} 204-\mathrm{H} 20 \mathrm{O}$ & 0.9600 \\
\hline $\mathrm{C} 1^{\prime}-\mathrm{C} 2^{\prime}$ & $1.399(9)$ & C204-H20P & 0.9600 \\
\hline $\mathrm{C} 1^{\prime}-\mathrm{C} 6^{\prime}$ & $1.391(8)$ & $\mathrm{C} 205-\mathrm{H} 20 \mathrm{R}$ & 0.9700 \\
\hline $\mathrm{C} 2^{\prime}-\mathrm{C} 3^{\prime}$ & $1.403(8)$ & $\mathrm{C} 205-\mathrm{H} 20 \mathrm{~S}$ & 0.9700 \\
\hline $\mathrm{C} 3^{\prime}-\mathrm{C} 4^{\prime}$ & $1.407(8)$ & $\mathrm{C} 206-\mathrm{H} 20 \mathrm{~T}$ & 0.9600 \\
\hline $\mathrm{C} 4^{\prime}-\mathrm{C} 5^{\prime}$ & $1.402(9)$ & $\mathrm{C} 206-\mathrm{H} 20 \mathrm{U}$ & 0.9600 \\
\hline $\mathrm{C} 5^{\prime}-\mathrm{C} 6^{\prime}$ & $1.383(8)$ & $\mathrm{C} 206-\mathrm{H} 20 \mathrm{~V}$ & 0.9600 \\
\hline $\mathrm{C} 6^{\prime}-\mathrm{C} 7^{\prime}$ & $1.535(10)$ & $\mathrm{C} 207-\mathrm{H} 20 \mathrm{Y}$ & 0.9700 \\
\hline $\mathrm{C} 7^{\prime}-\mathrm{C} 8^{\prime}$ & $1.519(13)$ & C207-H20Z & 0.9700 \\
\hline $\mathrm{C} 7^{\prime}-\mathrm{C} 9^{\prime}$ & $1.533(12)$ & $\mathrm{C} 208-\mathrm{H} 2 \mathrm{QW}$ & 0.9600 \\
\hline $\mathrm{C} 7^{\prime}-\mathrm{C} 10^{\prime}$ & $1.509(12)$ & C208-H20Q & 0.9600 \\
\hline $\mathrm{C} 11^{\prime}-\mathrm{C} 12^{\prime}$ & $1.397(7)$ & $\mathrm{C} 208-\mathrm{H} 20 \mathrm{~W}$ & 0.9600 \\
\hline $\mathrm{C} 2-\mathrm{S} 1-\mathrm{C} 34$ & $97.9(3)$ & $\mathrm{C} 22^{\prime}-\mathrm{C} 23^{\prime}-\mathrm{C} 24^{\prime}$ & $117.4(5)$ \\
\hline $\mathrm{C} 4-\mathrm{S} 2-\mathrm{C} 12$ & $105.3(3)$ & $\mathrm{C} 23^{\prime}-\mathrm{C} 24^{\prime}-\mathrm{C} 25^{\prime}$ & $120.8(5)$ \\
\hline $\mathrm{C} 14-\mathrm{S} 3-\mathrm{C} 22$ & $102.7(2)$ & $\mathrm{S} 4^{\prime}-\mathrm{C} 24^{\prime}-\mathrm{C} 25^{\prime}$ & $119.5(4)$ \\
\hline $\mathrm{C} 24-\mathrm{S} 4-\mathrm{C} 32$ & $97.0(2)$ & $\mathrm{S} 4^{\prime}-\mathrm{C} 24^{\prime}-\mathrm{C} 23^{\prime}$ & $119.5(4)$ \\
\hline $\mathrm{C} 2{ }^{\prime}-\mathrm{S} 1^{\prime}-\mathrm{C} 34^{\prime}$ & $97.7(3)$ & $\mathrm{C} 24^{\prime}-\mathrm{C} 25^{\prime}-\mathrm{C} 26^{\prime}$ & $122.1(5)$ \\
\hline $\mathrm{C} 4^{\prime}-\mathrm{S} 2^{\prime}-\mathrm{C} 12^{\prime}$ & $104.9(3)$ & $\mathrm{C} 21^{\prime}-\mathrm{C} 26^{\prime}-\mathrm{C} 25^{\prime}$ & $116.4(5)$ \\
\hline $\mathrm{C} 14^{\prime}-\mathrm{S} 3^{\prime}-\mathrm{C} 22^{\prime}$ & $102.5(3)$ & $\mathrm{C} 21^{\prime}-\mathrm{C} 26^{\prime}-\mathrm{C} 27^{\prime}$ & $121.1(5)$ \\
\hline $\mathrm{C} 24^{\prime}-\mathrm{S} 4^{\prime}-\mathrm{C} 32^{\prime}$ & $97.5(2)$ & $\mathrm{C} 25^{\prime}-\mathrm{C} 26^{\prime}-\mathrm{C} 27^{\prime}$ & $122.6(5)$ \\
\hline $\mathrm{C} 33-\mathrm{O} 4-\mathrm{C} 41$ & $112.1(3)$ & $\mathrm{C} 28^{\prime}-\mathrm{C} 27^{\prime}-\mathrm{C} 29^{\prime}$ & $109.6(6)$ \\
\hline $\mathrm{C} 3-\mathrm{O} 1-\mathrm{H} 1 \mathrm{O}$ & 110.00 & $\mathrm{C} 28^{\prime}-\mathrm{C} 27^{\prime}-\mathrm{C} 30^{\prime}$ & $106.5(6)$ \\
\hline $\mathrm{C} 13-\mathrm{O} 2-\mathrm{H} 2 \mathrm{O}$ & 109.00 & $\mathrm{C} 29^{\prime}-\mathrm{C} 27^{\prime}-\mathrm{C} 30^{\prime}$ & $108.9(6)$ \\
\hline $\mathrm{C} 23-\mathrm{O} 3-\mathrm{H} 3 \mathrm{O}$ & 109.00 & $\mathrm{C} 26^{\prime}-\mathrm{C} 27^{\prime}-\mathrm{C} 30^{\prime}$ & $113.0(5)$ \\
\hline $\mathrm{C} 2-\mathrm{C} 1-\mathrm{C} 6$ & $120.9(5)$ & $\mathrm{C} 26^{\prime}-\mathrm{C} 27^{\prime}-\mathrm{C} 28^{\prime}$ & $110.2(5)$ \\
\hline $\mathrm{S} 1-\mathrm{C} 2-\mathrm{C} 3$ & $119.8(4)$ & $\mathrm{C} 26^{\prime}-\mathrm{C} 27^{\prime}-\mathrm{C} 29^{\prime}$ & $108.7(5)$ \\
\hline $\mathrm{C} 1-\mathrm{C} 2-\mathrm{C} 3$ & $121.8(5)$ & $\mathrm{C} 32^{\prime}-\mathrm{C} 31^{\prime}-\mathrm{C} 36^{\prime}$ & $121.1(5)$ \\
\hline $\mathrm{S} 1-\mathrm{C} 2-\mathrm{C} 1$ & $118.4(4)$ & $\mathrm{C} 31^{\prime}-\mathrm{C} 32^{\prime}-\mathrm{C} 33^{\prime}$ & $120.7(4)$ \\
\hline $\mathrm{O} 1-\mathrm{C} 3-\mathrm{C} 4$ & $124.6(5)$ & $\mathrm{S} 4^{\prime}-\mathrm{C} 32^{\prime}-\mathrm{C} 33^{\prime}$ & $119.8(3)$ \\
\hline $\mathrm{O} 1-\mathrm{C} 3-\mathrm{C} 2$ & $118.5(5)$ & $\mathrm{S} 4^{\prime}-\mathrm{C} 32^{\prime}-\mathrm{C} 31^{\prime}$ & $119.3(4)$ \\
\hline $\mathrm{C} 2-\mathrm{C} 3-\mathrm{C} 4$ & $116.9(5)$ & $\mathrm{C} 32^{\prime}-\mathrm{C} 33^{\prime}-\mathrm{C} 34^{\prime}$ & $118.9(4)$ \\
\hline $\mathrm{S} 2-\mathrm{C} 4-\mathrm{C} 3$ & $120.6(4)$ & $\mathrm{O} 4^{\prime}-\mathrm{C} 33^{\prime}-\mathrm{C} 34^{\prime}$ & $119.8(4)$ \\
\hline $\mathrm{C} 3-\mathrm{C} 4-\mathrm{C} 5$ & $120.8(5)$ & $\mathrm{O} 4^{\prime}-\mathrm{C} 33^{\prime}-\mathrm{C} 32^{\prime}$ & $121.3(4)$ \\
\hline $\mathrm{S} 2-\mathrm{C} 4-\mathrm{C} 5$ & $118.2(4)$ & $\mathrm{S} 1^{\prime}-\mathrm{C} 34^{\prime}-\mathrm{C} 33^{\prime}$ & $119.6(3)$ \\
\hline $\mathrm{C} 33^{\prime}-\mathrm{O} 4^{\prime}-\mathrm{C} 41^{\prime}$ & $112.2(3)$ & $\mathrm{S} 1^{\prime}-\mathrm{C} 34^{\prime}-\mathrm{C} 35^{\prime}$ & $120.1(4)$ \\
\hline $\mathrm{C} 4-\mathrm{C} 5-\mathrm{C} 6$ & $122.2(5)$ & $\mathrm{C} 33^{\prime}-\mathrm{C} 34^{\prime}-\mathrm{C} 35^{\prime}$ & $120.0(4)$ \\
\hline $\mathrm{C} 5-\mathrm{C} 6-\mathrm{C} 7$ & $120.8(5)$ & $\mathrm{C} 34^{\prime}-\mathrm{C} 35^{\prime}-\mathrm{C} 36^{\prime}$ & $121.8(5)$ \\
\hline $\mathrm{C} 1-\mathrm{C} 6-\mathrm{C} 5$ & $117.3(5)$ & $\mathrm{C} 35^{\prime}-\mathrm{C} 36^{\prime}-\mathrm{C} 37^{\prime}$ & $120.9(5)$ \\
\hline $\mathrm{C} 1-\mathrm{C} 6-\mathrm{C} 7$ & $121.9(5)$ & $\mathrm{C} 31^{\prime}-\mathrm{C} 36^{\prime}-\mathrm{C} 35^{\prime}$ & $117.5(4)$ \\
\hline $\mathrm{C} 8-\mathrm{C} 7-\mathrm{C} 9$ & $110.2(8)$ & $\mathrm{C} 31^{\prime}-\mathrm{C} 36^{\prime}-\mathrm{C} 37^{\prime}$ & $121.5(5)$ \\
\hline $\mathrm{C} 6-\mathrm{C} 7-\mathrm{C} 9$ & $107.8(5)$ & $\mathrm{C} 36^{\prime}-\mathrm{C} 37^{\prime}-\mathrm{C} 38^{\prime}$ & $112.7(5)$ \\
\hline
\end{tabular}




\begin{tabular}{|c|c|c|c|}
\hline $\mathrm{C} 6-\mathrm{C} 7-\mathrm{C} 10$ & $109.3(7)$ & $\mathrm{C} 36^{\prime}-\mathrm{C} 37^{\prime}-\mathrm{C} 39^{\prime}$ & $109.2(5)$ \\
\hline $\mathrm{C} 8-\mathrm{C} 7-\mathrm{C} 10$ & $109.0(9)$ & $\mathrm{C} 36^{\prime}-\mathrm{C} 37^{\prime}-\mathrm{C} 40^{\prime}$ & $109.7(5)$ \\
\hline $\mathrm{C} 9-\mathrm{C} 7-\mathrm{C} 10$ & $108.6(8)$ & $\mathrm{C} 39^{\prime}-\mathrm{C} 37^{\prime}-\mathrm{C} 40^{\prime}$ & $110.2(7)$ \\
\hline $\mathrm{C} 6-\mathrm{C} 7-\mathrm{C} 8$ & $112.0(7)$ & $\mathrm{C} 38^{\prime}-\mathrm{C} 37^{\prime}-\mathrm{C} 39^{\prime}$ & $108.2(8)$ \\
\hline $\mathrm{C} 12-\mathrm{C} 11-\mathrm{C} 16$ & $122.2(5)$ & $\mathrm{C} 38^{\prime}-\mathrm{C} 37^{\prime}-\mathrm{C} 40^{\prime}$ & $106.9(7)$ \\
\hline $\mathrm{S} 2-\mathrm{C} 12-\mathrm{C} 11$ & $118.9(4)$ & $\mathrm{O} 4^{\prime}-\mathrm{C} 41^{\prime}-\mathrm{C} 42^{\prime}$ & $108.1(4)$ \\
\hline $\mathrm{S} 2-\mathrm{C} 12-\mathrm{C} 13$ & $119.6(3)$ & $\mathrm{O} 5^{\prime}-\mathrm{C} 42^{\prime}-\mathrm{C} 41^{\prime}$ & $120.5(4)$ \\
\hline $\mathrm{C} 11-\mathrm{C} 12-\mathrm{C} 13$ & $121.2(4)$ & $\mathrm{O}^{\prime}-\mathrm{C} 42^{\prime}-\mathrm{C} 43^{\prime}$ & $121.6(4)$ \\
\hline $\mathrm{O} 2-\mathrm{C} 13-\mathrm{C} 14$ & $122.5(5)$ & $\mathrm{C} 41^{\prime}-\mathrm{C} 42^{\prime}-\mathrm{C} 43^{\prime}$ & $117.9(4)$ \\
\hline $\mathrm{O} 2-\mathrm{C} 13-\mathrm{C} 12$ & $122.1(5)$ & $\mathrm{C} 42^{\prime}-\mathrm{C} 43^{\prime}-\mathrm{C} 44^{\prime}$ & $117.5(5)$ \\
\hline $\mathrm{C} 12-\mathrm{C} 13-\mathrm{C} 14$ & $115.5(4)$ & $\mathrm{C} 42^{\prime}-\mathrm{C} 43^{\prime}-\mathrm{C} 48^{\prime}$ & $122.9(4)$ \\
\hline $\mathrm{C} 13-\mathrm{C} 14-\mathrm{C} 15$ & $121.3(4)$ & $\mathrm{C} 44^{\prime}-\mathrm{C} 43^{\prime}-\mathrm{C} 48^{\prime}$ & $119.5(4)$ \\
\hline $\mathrm{S} 3-\mathrm{C} 14-\mathrm{C} 13$ & $119.8(3)$ & $\mathrm{C} 43^{\prime}-\mathrm{C} 44^{\prime}-\mathrm{C} 45^{\prime}$ & $120.0(5)$ \\
\hline $\mathrm{S} 3-\mathrm{C} 14-\mathrm{C} 15$ & $118.9(4)$ & $\mathrm{C} 44^{\prime}-\mathrm{C} 45^{\prime}-\mathrm{C} 46^{\prime}$ & $120.0(4)$ \\
\hline $\mathrm{C} 14-\mathrm{C} 15-\mathrm{C} 16$ & $122.6(5)$ & $\mathrm{C} 45^{\prime}-\mathrm{C} 46^{\prime}-\mathrm{C} 47^{\prime}$ & $119.7(5)$ \\
\hline $\mathrm{C} 11-\mathrm{C} 16-\mathrm{C} 15$ & $116.5(4)$ & $\mathrm{C} 46^{\prime}-\mathrm{C} 47^{\prime}-\mathrm{C} 48^{\prime}$ & $120.6(5)$ \\
\hline $\mathrm{C} 15-\mathrm{C} 16-\mathrm{C} 17$ & $121.4(5)$ & $\mathrm{C} 43^{\prime}-\mathrm{C} 48^{\prime}-\mathrm{C} 47^{\prime}$ & $120.2(4)$ \\
\hline $\mathrm{C} 11-\mathrm{C} 16-\mathrm{C} 17$ & $122.0(5)$ & $\mathrm{C} 105-\mathrm{N} 1-\mathrm{C} 107$ & $104.8(9)$ \\
\hline $\mathrm{C} 16-\mathrm{C} 17-\mathrm{C} 19$ & $109.4(5)$ & $\mathrm{C} 101-\mathrm{N} 1-\mathrm{C} 107$ & $108.3(8)$ \\
\hline $\mathrm{C} 16-\mathrm{C} 17-\mathrm{C} 20$ & $109.0(5)$ & $\mathrm{C} 101-\mathrm{N} 1-\mathrm{C} 103$ & $109.7(8)$ \\
\hline $\mathrm{C} 18-\mathrm{C} 17-\mathrm{C} 20$ & $107.2(6)$ & $\mathrm{C} 101-\mathrm{N} 1-\mathrm{C} 105$ & $105.6(9)$ \\
\hline $\mathrm{C} 16-\mathrm{C} 17-\mathrm{C} 18$ & $113.0(5)$ & $\mathrm{C} 103-\mathrm{N} 1-\mathrm{C} 105$ & $118.9(10)$ \\
\hline $\mathrm{C} 19-\mathrm{C} 17-\mathrm{C} 20$ & $109.0(6)$ & $\mathrm{C} 103-\mathrm{N} 1-\mathrm{C} 107$ & $109.1(8)$ \\
\hline $\mathrm{C} 18-\mathrm{C} 17-\mathrm{C} 19$ & $109.2(7)$ & $\mathrm{C} 6^{\prime}-\mathrm{C} 1^{\prime}-\mathrm{H} 1^{\prime}$ & 119.00 \\
\hline $\mathrm{C} 22-\mathrm{C} 21-\mathrm{C} 26$ & $121.9(5)$ & $\mathrm{C} 2^{\prime}-\mathrm{C} 1^{\prime}-\mathrm{H} 1^{\prime}$ & 119.00 \\
\hline $\mathrm{C} 21-\mathrm{C} 22-\mathrm{C} 23$ & $121.6(5)$ & $\mathrm{C} 4^{\prime}-\mathrm{C} 5^{\prime}-\mathrm{H} 5^{\prime}$ & 119.00 \\
\hline $\mathrm{S} 3-\mathrm{C} 22-\mathrm{C} 21$ & $118.7(4)$ & $\mathrm{C} 6^{\prime}-\mathrm{C} 5^{\prime}-\mathrm{H} 5^{\prime}$ & 119.00 \\
\hline $\mathrm{S} 3-\mathrm{C} 22-\mathrm{C} 23$ & $119.6(4)$ & $\mathrm{H} 8 \mathrm{~A}^{\prime}-\mathrm{C} 8^{\prime}-\mathrm{H}_{8} \mathrm{C}^{\prime}$ & 109.00 \\
\hline $\mathrm{O} 3-\mathrm{C} 23-\mathrm{C} 24$ & $119.1(5)$ & $\mathrm{C} 7^{\prime}-\mathrm{C} 8^{\prime}-\mathrm{H} 8 \mathrm{~A}^{\prime}$ & 110.00 \\
\hline $\mathrm{O} 3-\mathrm{C} 23-\mathrm{C} 22$ & $124.5(5)$ & $\mathrm{C}^{\prime}-\mathrm{C} 8^{\prime}-\mathrm{H} 8 \mathrm{~B}^{\prime}$ & 110.00 \\
\hline $\mathrm{C} 22-\mathrm{C} 23-\mathrm{C} 24$ & $116.4(5)$ & $\mathrm{C}^{\prime}-\mathrm{C} 8^{\prime}-\mathrm{H} 8 \mathrm{C}^{\prime}$ & 109.00 \\
\hline $\mathrm{S} 4-\mathrm{C} 24-\mathrm{C} 23$ & $119.4(4)$ & $\mathrm{H} 8 \mathrm{~A}^{\prime}-\mathrm{C} 8^{\prime}-\mathrm{H} 8 \mathrm{~B}^{\prime}$ & 109.00 \\
\hline $\mathrm{C} 23-\mathrm{C} 24-\mathrm{C} 25$ & $121.5(5)$ & $\mathrm{H} 8 \mathrm{~B}^{\prime}-\mathrm{C} 8^{\prime}-\mathrm{H} 8 \mathrm{C}^{\prime}$ & 109.00 \\
\hline $\mathrm{S} 4-\mathrm{C} 24-\mathrm{C} 25$ & $119.1(4)$ & $\mathrm{H} 9 \mathrm{~A}^{\prime}-\mathrm{C} 9^{\prime}-\mathrm{H} 9 \mathrm{C}^{\prime}$ & 110.00 \\
\hline $\mathrm{C} 24-\mathrm{C} 25-\mathrm{C} 26$ & $121.8(5)$ & $\mathrm{C}^{\prime}-\mathrm{C} 9^{\prime}-\mathrm{H}^{\prime} \mathrm{B}^{\prime}$ & 109.00 \\
\hline $\mathrm{C} 21-\mathrm{C} 26-\mathrm{C} 25$ & $116.8(5)$ & $\mathrm{H} 9 \mathrm{~B}^{\prime}-\mathrm{C}^{\prime}-\mathrm{H} 9 \mathrm{C}^{\prime}$ & 110.00 \\
\hline $\mathrm{C} 21-\mathrm{C} 26-\mathrm{C} 27$ & $119.8(5)$ & $\mathrm{C} 7^{\prime}-\mathrm{C} 9^{\prime}-\mathrm{H} 9 \mathrm{~A}^{\prime}$ & 109.00 \\
\hline $\mathrm{C} 25-\mathrm{C} 26-\mathrm{C} 27$ & $123.4(5)$ & $\mathrm{C} 7^{\prime}-\mathrm{C} 9^{\prime}-\mathrm{H} 9 \mathrm{C}^{\prime}$ & 109.00 \\
\hline $\mathrm{C} 26-\mathrm{C} 27-\mathrm{C} 28$ & $109.5(6)$ & $\mathrm{H}^{\prime} \mathrm{A}^{\prime}-\mathrm{C} 9^{\prime}-\mathrm{H}^{\prime} \mathrm{B}^{\prime}$ & 110.00 \\
\hline $\mathrm{C} 26-\mathrm{C} 27-\mathrm{C} 29$ & $110.4(6)$ & $\mathrm{C}^{\prime}-\mathrm{C} 10^{\prime}-\mathrm{H} 10 \mathrm{E}$ & 109.00 \\
\hline $\mathrm{C} 26-\mathrm{C} 27-\mathrm{C} 30$ & $111.8(7)$ & $\mathrm{C} 7^{\prime}-\mathrm{C} 10^{\prime}-\mathrm{H} 10 \mathrm{D}$ & 110.00 \\
\hline $\mathrm{C} 28-\mathrm{C} 27-\mathrm{C} 29$ & $109.4(7)$ & $\mathrm{H} 10 \mathrm{D}-\mathrm{C} 10^{\prime}-\mathrm{H} 10 \mathrm{~F}$ & 109.00 \\
\hline $\mathrm{C} 28-\mathrm{C} 27-\mathrm{C} 30$ & $108.9(8)$ & $\mathrm{C} 7^{\prime}-\mathrm{C} 10^{\prime}-\mathrm{H} 10 \mathrm{~F}$ & 110.00 \\
\hline $\mathrm{C} 29-\mathrm{C} 27-\mathrm{C} 30$ & $106.9(8)$ & $\mathrm{H} 10 \mathrm{D}-\mathrm{C} 10^{\prime}-\mathrm{H} 10 \mathrm{E}$ & 109.00 \\
\hline $\mathrm{C} 32-\mathrm{C} 31-\mathrm{C} 36$ & $121.8(4)$ & $\mathrm{H} 10 \mathrm{E}-\mathrm{C} 10^{\prime}-\mathrm{H} 10 \mathrm{~F}$ & 109.00 \\
\hline $\mathrm{S} 4-\mathrm{C} 32-\mathrm{C} 33$ & $119.6(3)$ & $\mathrm{C} 16^{\prime}-\mathrm{C} 11^{\prime}-\mathrm{H} 11^{\prime}$ & 119.00 \\
\hline $\mathrm{S} 4-\mathrm{C} 32-\mathrm{C} 31$ & $120.0(3)$ & $\mathrm{C} 12^{\prime}-\mathrm{C} 11^{\prime}-\mathrm{H} 11^{\prime}$ & 119.00 \\
\hline $\mathrm{C} 31-\mathrm{C} 32-\mathrm{C} 33$ & $120.1(4)$ & $\mathrm{C} 14^{\prime}-\mathrm{C} 15^{\prime}-\mathrm{H} 15^{\prime}$ & 118.00 \\
\hline
\end{tabular}




\begin{tabular}{|c|c|}
\hline $\mathrm{O} 4-\mathrm{C} 33-\mathrm{C} 34$ & $120.2(5)$ \\
\hline $\mathrm{C} 32-\mathrm{C} 33-\mathrm{C} 34$ & $119.1(4)$ \\
\hline $\mathrm{O} 4-\mathrm{C} 33-\mathrm{C} 32$ & $120.8(4)$ \\
\hline $\mathrm{S} 1-\mathrm{C} 34-\mathrm{C} 33$ & $120.0(3)$ \\
\hline $\mathrm{C} 33-\mathrm{C} 34-\mathrm{C} 35$ & $120.4(5)$ \\
\hline $\mathrm{S} 1-\mathrm{C} 34-\mathrm{C} 35$ & $119.4(4)$ \\
\hline $\mathrm{C} 34-\mathrm{C} 35-\mathrm{C} 36$ & $121.0(5)$ \\
\hline $\mathrm{C} 35-\mathrm{C} 36-\mathrm{C} 37$ & $121.0(5)$ \\
\hline C31-C36-C37 & $121.4(5)$ \\
\hline $\mathrm{C} 31-\mathrm{C} 36-\mathrm{C} 35$ & $117.6(4)$ \\
\hline $\mathrm{C} 36-\mathrm{C} 37-\mathrm{C} 40$ & $110.4(4)$ \\
\hline $\mathrm{C} 39-\mathrm{C} 37-\mathrm{C} 40$ & $108.9(6)$ \\
\hline $\mathrm{C} 36-\mathrm{C} 37-\mathrm{C} 39$ & $109.4(5)$ \\
\hline $\mathrm{C} 38-\mathrm{C} 37-\mathrm{C} 39$ & $108.2(6)$ \\
\hline $\mathrm{C} 38-\mathrm{C} 37-\mathrm{C} 40$ & $108.0(7)$ \\
\hline $\mathrm{C} 36-\mathrm{C} 37-\mathrm{C} 38$ & $111.9(5)$ \\
\hline $\mathrm{O} 4-\mathrm{C} 41-\mathrm{C} 42$ & $108.2(4)$ \\
\hline $\mathrm{O} 5-\mathrm{C} 42-\mathrm{C} 43$ & $121.8(4)$ \\
\hline $\mathrm{O} 5-\mathrm{C} 42-\mathrm{C} 41$ & $120.3(4)$ \\
\hline $\mathrm{C} 41-\mathrm{C} 42-\mathrm{C} 43$ & $117.8(4)$ \\
\hline $\mathrm{C} 42-\mathrm{C} 43-\mathrm{C} 44$ & $118.2(4)$ \\
\hline $\mathrm{C} 42-\mathrm{C} 43-\mathrm{C} 48$ & $122.7(4)$ \\
\hline $\mathrm{C} 44-\mathrm{C} 43-\mathrm{C} 48$ & $119.1(4)$ \\
\hline $\mathrm{C} 43-\mathrm{C} 44-\mathrm{C} 45$ & $120.5(5)$ \\
\hline $\mathrm{C} 44-\mathrm{C} 45-\mathrm{C} 46$ & $119.8(5)$ \\
\hline $\mathrm{C} 45-\mathrm{C} 46-\mathrm{C} 47$ & $120.3(5)$ \\
\hline $\mathrm{C} 46-\mathrm{C} 47-\mathrm{C} 48$ & $120.2(5)$ \\
\hline $\mathrm{C} 43-\mathrm{C} 48-\mathrm{C} 47$ & $120.1(4)$ \\
\hline $\mathrm{C} 6-\mathrm{C} 1-\mathrm{H} 1$ & 120.00 \\
\hline $\mathrm{C} 2-\mathrm{C} 1-\mathrm{H} 1$ & 120.00 \\
\hline $\mathrm{C}^{\prime}-\mathrm{O} 1^{\prime}-\mathrm{H} 6 \mathrm{O}$ & 109.00 \\
\hline $\mathrm{C} 13^{\prime}-\mathrm{O} 2^{\prime}-\mathrm{H} 7 \mathrm{O}$ & 109.00 \\
\hline $\mathrm{C}^{\prime} 3^{\prime}-\mathrm{O}^{\prime}-\mathrm{H} 8 \mathrm{O}$ & 109.00 \\
\hline $\mathrm{C} 4-\mathrm{C} 5-\mathrm{H} 5$ & 119.00 \\
\hline $\mathrm{C} 6-\mathrm{C} 5-\mathrm{H} 5$ & 119.00 \\
\hline $\mathrm{H} 8 \mathrm{~A}-\mathrm{C} 8-\mathrm{H} 8 \mathrm{C}$ & 109.00 \\
\hline $\mathrm{C} 7-\mathrm{C} 8-\mathrm{H} 8 \mathrm{~A}$ & 109.00 \\
\hline $\mathrm{H} 8 \mathrm{~A}-\mathrm{C} 8-\mathrm{H} 8 \mathrm{~B}$ & 109.00 \\
\hline $\mathrm{H} 8 \mathrm{~B}-\mathrm{C} 8-\mathrm{H} 8 \mathrm{C}$ & 110.00 \\
\hline $\mathrm{C} 7-\mathrm{C} 8-\mathrm{H} 8 \mathrm{~B}$ & 109.00 \\
\hline $\mathrm{C} 7-\mathrm{C} 8-\mathrm{H} 8 \mathrm{C}$ & 109.00 \\
\hline $\mathrm{H} 9 \mathrm{~B}-\mathrm{C} 9-\mathrm{H} 9 \mathrm{C}$ & 110.00 \\
\hline $\mathrm{C} 7-\mathrm{C} 9-\mathrm{H} 9 \mathrm{C}$ & 110.00 \\
\hline $\mathrm{C} 7-\mathrm{C} 9-\mathrm{H} 9 \mathrm{~B}$ & 109.00 \\
\hline $\mathrm{H} 9 \mathrm{~A}-\mathrm{C} 9-\mathrm{H} 9 \mathrm{C}$ & 109.00 \\
\hline $\mathrm{H} 9 \mathrm{~A}-\mathrm{C} 9-\mathrm{H} 9 \mathrm{~B}$ & 109.00 \\
\hline $\mathrm{C} 7-\mathrm{C} 9-\mathrm{H} 9 \mathrm{~A}$ & 109.00 \\
\hline $\mathrm{H} 10 \mathrm{~A}-\mathrm{C} 10-\mathrm{H} 10 \mathrm{C}$ & 109.00 \\
\hline
\end{tabular}

\begin{tabular}{|c|c|}
\hline $\mathrm{C} 16^{\prime}-\mathrm{C} 15^{\prime}-\mathrm{H} 15^{\prime}$ & 118.00 \\
\hline $\mathrm{H} 18 \mathrm{E}-\mathrm{C} 18^{\prime}-\mathrm{H} 18 \mathrm{~F}$ & 109.00 \\
\hline $\mathrm{C} 17^{\prime}-\mathrm{C} 18^{\prime}-\mathrm{H} 18 \mathrm{E}$ & 110.00 \\
\hline $\mathrm{C} 17^{\prime}-\mathrm{C} 18^{\prime}-\mathrm{H} 18 \mathrm{D}$ & 109.00 \\
\hline $\mathrm{H} 18 \mathrm{D}-\mathrm{C} 18^{\prime}-\mathrm{H} 18 \mathrm{~F}$ & 109.00 \\
\hline $\mathrm{C} 17^{\prime}-\mathrm{C} 18^{\prime}-\mathrm{H} 18 \mathrm{~F}$ & 110.00 \\
\hline $\mathrm{H} 18 \mathrm{D}-\mathrm{C} 18^{\prime}-\mathrm{H} 18 \mathrm{E}$ & 109.00 \\
\hline C17'-C19'-H19D & 109.00 \\
\hline H19D $-\mathrm{C} 19^{\prime}-\mathrm{H} 19 \mathrm{E}$ & 109.00 \\
\hline $\mathrm{C} 17^{\prime}-\mathrm{C} 19^{\prime}-\mathrm{H} 19 \mathrm{E}$ & 109.00 \\
\hline $\mathrm{C} 17^{\prime}-\mathrm{C} 19^{\prime}-\mathrm{H} 19 \mathrm{~F}$ & 110.00 \\
\hline H19D $-\mathrm{C} 19^{\prime}-\mathrm{H} 19 \mathrm{~F}$ & 109.00 \\
\hline $\mathrm{H} 19 \mathrm{E}-\mathrm{C} 19^{\prime}-\mathrm{H} 19 \mathrm{~F}$ & 110.00 \\
\hline $\mathrm{C} 17^{\prime}-\mathrm{C} 20^{\prime}-\mathrm{H} 20 \mathrm{E}$ & 110.00 \\
\hline $\mathrm{C} 17^{\prime}-\mathrm{C} 20^{\prime}-\mathrm{H} 20 \mathrm{D}$ & 109.00 \\
\hline $\mathrm{H} 20 \mathrm{E}-\mathrm{C} 20^{\prime}-\mathrm{H} 20 \mathrm{~F}$ & 110.00 \\
\hline $\mathrm{C} 17^{\prime}-\mathrm{C} 20^{\prime}-\mathrm{H} 20 \mathrm{~F}$ & 109.00 \\
\hline $\mathrm{H} 20 \mathrm{D}-\mathrm{C} 20^{\prime}-\mathrm{H} 20 \mathrm{E}$ & 109.00 \\
\hline $\mathrm{H} 20 \mathrm{D}-\mathrm{C} 20^{\prime}-\mathrm{H} 20 \mathrm{~F}$ & 109.00 \\
\hline $\mathrm{C} 22^{\prime}-\mathrm{C} 21^{\prime}-\mathrm{H} 21^{\prime}$ & 119.00 \\
\hline $\mathrm{C} 26^{\prime}-\mathrm{C} 21^{\prime}-\mathrm{H} 21^{\prime}$ & 119.00 \\
\hline $\mathrm{C} 24^{\prime}-\mathrm{C} 25^{\prime}-\mathrm{H} 25^{\prime}$ & 119.00 \\
\hline $\mathrm{C} 26^{\prime}-\mathrm{C} 25^{\prime}-\mathrm{H} 25^{\prime}$ & 119.00 \\
\hline $\mathrm{C} 27^{\prime}-\mathrm{C} 28^{\prime}-\mathrm{H} 28 \mathrm{E}$ & 109.00 \\
\hline $\mathrm{C} 27^{\prime}-\mathrm{C} 28^{\prime}-\mathrm{H} 28 \mathrm{D}$ & 109.00 \\
\hline $\mathrm{H} 28 \mathrm{D}-\mathrm{C} 28^{\prime}-\mathrm{H} 28 \mathrm{~F}$ & 110.00 \\
\hline $\mathrm{C} 27^{\prime}-\mathrm{C} 28^{\prime}-\mathrm{H} 28 \mathrm{~F}$ & 110.00 \\
\hline $\mathrm{H} 28 \mathrm{D}-\mathrm{C} 28^{\prime}-\mathrm{H} 28 \mathrm{E}$ & 109.00 \\
\hline $\mathrm{H} 28 \mathrm{E}-\mathrm{C} 28^{\prime}-\mathrm{H} 28 \mathrm{~F}$ & 109.00 \\
\hline $\mathrm{C} 27^{\prime}-\mathrm{C} 29^{\prime}-\mathrm{H} 29 \mathrm{~F}$ & 109.00 \\
\hline $\mathrm{C} 27^{\prime}-\mathrm{C} 29^{\prime}-\mathrm{H} 29 \mathrm{D}$ & 110.00 \\
\hline $\mathrm{C} 27^{\prime}-\mathrm{C} 29^{\prime}-\mathrm{H} 29 \mathrm{E}$ & 109.00 \\
\hline $\mathrm{H} 29 \mathrm{D}-\mathrm{C} 29^{\prime}-\mathrm{H} 29 \mathrm{~F}$ & 109.00 \\
\hline $\mathrm{H} 29 \mathrm{E}-\mathrm{C} 29^{\prime}-\mathrm{H} 29 \mathrm{~F}$ & 109.00 \\
\hline $\mathrm{H} 29 \mathrm{D}-\mathrm{C} 29^{\prime}-\mathrm{H} 29 \mathrm{E}$ & 110.00 \\
\hline $\mathrm{H} 30 \mathrm{D}-\mathrm{C} 30^{\prime}-\mathrm{H} 30 \mathrm{E}$ & 109.00 \\
\hline $\mathrm{H} 30 \mathrm{D}-\mathrm{C} 30^{\prime}-\mathrm{H} 30 \mathrm{~F}$ & 109.00 \\
\hline $\mathrm{C} 27^{\prime}-\mathrm{C} 30^{\prime}-\mathrm{H} 30 \mathrm{D}$ & 110.00 \\
\hline $\mathrm{C} 27^{\prime}-\mathrm{C} 30^{\prime}-\mathrm{H} 30 \mathrm{E}$ & 110.00 \\
\hline $\mathrm{C} 27^{\prime}-\mathrm{C} 30^{\prime}-\mathrm{H} 30 \mathrm{~F}$ & 109.00 \\
\hline $\mathrm{H} 30 \mathrm{E}-\mathrm{C} 30^{\prime}-\mathrm{H} 30 \mathrm{~F}$ & 109.00 \\
\hline $\mathrm{C} 36^{\prime}-\mathrm{C} 31^{\prime}-\mathrm{H} 31^{\prime}$ & 119.00 \\
\hline $\mathrm{C} 32^{\prime}-\mathrm{C} 31^{\prime}-\mathrm{H} 31^{\prime}$ & 120.00 \\
\hline $\mathrm{C} 34^{\prime}-\mathrm{C} 35^{\prime}-\mathrm{H} 35^{\prime}$ & 119.00 \\
\hline $\mathrm{C} 36^{\prime}-\mathrm{C} 35^{\prime}-\mathrm{H} 35^{\prime}$ & 119.00 \\
\hline $\mathrm{C} 37^{\prime}-\mathrm{C} 38^{\prime}-\mathrm{H} 38 \mathrm{E}$ & 109.00 \\
\hline $\mathrm{C} 37^{\prime}-\mathrm{C} 38^{\prime}-\mathrm{H} 38 \mathrm{D}$ & 109.00 \\
\hline $\mathrm{C} 37^{\prime}-\mathrm{C} 38^{\prime}-\mathrm{H} 38 \mathrm{~F}$ & 110.00 \\
\hline
\end{tabular}




\begin{tabular}{|c|c|}
\hline $\mathrm{C} 7-\mathrm{C} 10-\mathrm{H} 10 \mathrm{C}$ & 109.00 \\
\hline $\mathrm{C} 7-\mathrm{C} 10-\mathrm{H} 10 \mathrm{~B}$ & 109.00 \\
\hline $\mathrm{C} 7-\mathrm{C} 10-\mathrm{H} 10 \mathrm{~A}$ & 109.00 \\
\hline $\mathrm{H} 10 \mathrm{~B}-\mathrm{C} 10-\mathrm{H} 10 \mathrm{C}$ & 110.00 \\
\hline $\mathrm{H} 10 \mathrm{~A}-\mathrm{C} 10-\mathrm{H} 10 \mathrm{~B}$ & 109.00 \\
\hline $\mathrm{C} 12-\mathrm{C} 11-\mathrm{H} 11$ & 119.00 \\
\hline $\mathrm{C} 16-\mathrm{C} 11-\mathrm{H} 11$ & 119.00 \\
\hline $\mathrm{C} 14-\mathrm{C} 15-\mathrm{H} 15$ & 119.00 \\
\hline $\mathrm{C} 16-\mathrm{C} 15-\mathrm{H} 15$ & 119.00 \\
\hline $\mathrm{C} 17-\mathrm{C} 18-\mathrm{H} 18 \mathrm{~B}$ & 110.00 \\
\hline $\mathrm{H} 18 \mathrm{~B}-\mathrm{C} 18-\mathrm{H} 18 \mathrm{C}$ & 110.00 \\
\hline $\mathrm{H} 18 \mathrm{~A}-\mathrm{C} 18-\mathrm{H} 18 \mathrm{C}$ & 109.00 \\
\hline C17-C18-H18A & 110.00 \\
\hline $\mathrm{C} 17-\mathrm{C} 18-\mathrm{H} 18 \mathrm{C}$ & 109.00 \\
\hline $\mathrm{H} 18 \mathrm{~A}-\mathrm{C} 18-\mathrm{H} 18 \mathrm{~B}$ & 109.00 \\
\hline C17-C19-H19B & 109.00 \\
\hline C17-C19-H19A & 109.00 \\
\hline $\mathrm{H} 19 \mathrm{~A}-\mathrm{C} 19-\mathrm{H} 19 \mathrm{~B}$ & 109.00 \\
\hline $\mathrm{H} 19 \mathrm{~A}-\mathrm{C} 19-\mathrm{H} 19 \mathrm{C}$ & 109.00 \\
\hline $\mathrm{C} 17-\mathrm{C} 19-\mathrm{H} 19 \mathrm{C}$ & 110.00 \\
\hline $\mathrm{H} 19 \mathrm{~B}-\mathrm{C} 19-\mathrm{H} 19 \mathrm{C}$ & 110.00 \\
\hline $\mathrm{H} 20 \mathrm{~B}-\mathrm{C} 20-\mathrm{H} 20 \mathrm{C}$ & 109.00 \\
\hline $\mathrm{C} 17-\mathrm{C} 20-\mathrm{H} 20 \mathrm{C}$ & 110.00 \\
\hline $\mathrm{C} 17-\mathrm{C} 20-\mathrm{H} 20 \mathrm{~B}$ & 110.00 \\
\hline $\mathrm{C} 17-\mathrm{C} 20-\mathrm{H} 20 \mathrm{~A}$ & 109.00 \\
\hline $\mathrm{H} 20 \mathrm{~A}-\mathrm{C} 20-\mathrm{H} 20 \mathrm{C}$ & 109.00 \\
\hline $\mathrm{H} 20 \mathrm{~A}-\mathrm{C} 20-\mathrm{H} 20 \mathrm{~B}$ & 109.00 \\
\hline $\mathrm{C} 22-\mathrm{C} 21-\mathrm{H} 21$ & 119.00 \\
\hline $\mathrm{C} 26-\mathrm{C} 21-\mathrm{H} 21$ & 119.00 \\
\hline $\mathrm{C} 26-\mathrm{C} 25-\mathrm{H} 25$ & 119.00 \\
\hline $\mathrm{C} 24-\mathrm{C} 25-\mathrm{H} 25$ & 119.00 \\
\hline $\mathrm{C} 27-\mathrm{C} 28-\mathrm{H} 28 \mathrm{~B}$ & 109.00 \\
\hline $\mathrm{H} 28 \mathrm{~A}-\mathrm{C} 28-\mathrm{H} 28 \mathrm{C}$ & 110.00 \\
\hline $\mathrm{C} 27-\mathrm{C} 28-\mathrm{H} 28 \mathrm{~A}$ & 109.00 \\
\hline $\mathrm{C} 27-\mathrm{C} 28-\mathrm{H} 28 \mathrm{C}$ & 109.00 \\
\hline $\mathrm{H} 28 \mathrm{~A}-\mathrm{C} 28-\mathrm{H} 28 \mathrm{~B}$ & 110.00 \\
\hline $\mathrm{H} 28 \mathrm{~B}-\mathrm{C} 28-\mathrm{H} 28 \mathrm{C}$ & 109.00 \\
\hline $\mathrm{C} 27-\mathrm{C} 29-\mathrm{H} 29 \mathrm{C}$ & 110.00 \\
\hline $\mathrm{C} 27-\mathrm{C} 29-\mathrm{H} 29 \mathrm{~B}$ & 109.00 \\
\hline $\mathrm{H} 29 \mathrm{~A}-\mathrm{C} 29-\mathrm{H} 29 \mathrm{C}$ & 109.00 \\
\hline $\mathrm{H} 29 \mathrm{~A}-\mathrm{C} 29-\mathrm{H} 29 \mathrm{~B}$ & 109.00 \\
\hline $\mathrm{H} 29 \mathrm{~B}-\mathrm{C} 29-\mathrm{H} 29 \mathrm{C}$ & 109.00 \\
\hline $\mathrm{C} 27-\mathrm{C} 29-\mathrm{H} 29 \mathrm{~A}$ & 110.00 \\
\hline $\mathrm{H} 30 \mathrm{~A}-\mathrm{C} 30-\mathrm{H} 30 \mathrm{C}$ & 109.00 \\
\hline $\mathrm{H} 30 \mathrm{~A}-\mathrm{C} 30-\mathrm{H} 30 \mathrm{~B}$ & 109.00 \\
\hline C27-C $30-\mathrm{H} 30 \mathrm{~B}$ & 110.00 \\
\hline $\mathrm{C} 27-\mathrm{C} 30-\mathrm{H} 30 \mathrm{C}$ & 109.00 \\
\hline $\mathrm{C} 27-\mathrm{C} 30-\mathrm{H} 30 \mathrm{~A}$ & 109.00 \\
\hline
\end{tabular}

109.00

110.00

109.00

109.00

109.00

109.00

109.00

109.00

110.00

109.00

109.00

109.00

109.00

109.00

109.00

110.00

110.00

110.00

108.00

110.00

120.00

120.00

120.00

120.00

120.00

120.00

120.00

120.00

120.00

120.00

115.9 (10)

$115.3(10)$

$115.1(12)$

113.0 (12)

109.5 (7)

111.7 (7)

$108.8(7)$

$111.0(7)$

104.3 (7)

$111.6(7)$

108.00

107.00

108.00

108.00

108.00

110.00

109.00

109.00 


\begin{tabular}{|c|c|c|c|}
\hline $\mathrm{H} 30 \mathrm{~B}-\mathrm{C} 30-\mathrm{H} 30 \mathrm{C}$ & 110.00 & $\mathrm{C} 101-\mathrm{C} 102-\mathrm{H} 10 \mathrm{~K}$ & 109.00 \\
\hline $\mathrm{C} 36-\mathrm{C} 31-\mathrm{H} 31$ & 119.00 & $\mathrm{H} 10 \mathrm{I}-\mathrm{C} 102-\mathrm{H} 10 \mathrm{~K}$ & 110.00 \\
\hline $\mathrm{C} 32-\mathrm{C} 31-\mathrm{H} 31$ & 119.00 & $\mathrm{H} 10 \mathrm{~J}-\mathrm{C} 102-\mathrm{H} 10 \mathrm{~K}$ & 109.00 \\
\hline $\mathrm{C} 36-\mathrm{C} 35-\mathrm{H} 35$ & 119.00 & $\mathrm{~N} 1-\mathrm{C} 103-\mathrm{H} 10 \mathrm{M}$ & 108.00 \\
\hline $\mathrm{C} 34-\mathrm{C} 35-\mathrm{H} 35$ & 119.00 & $\mathrm{~N} 1-\mathrm{C} 103-\mathrm{H} 10 \mathrm{~L}$ & 108.00 \\
\hline $\mathrm{C} 37-\mathrm{C} 38-\mathrm{H} 38 \mathrm{~B}$ & 109.00 & $\mathrm{C} 104-\mathrm{C} 103-\mathrm{H} 10 \mathrm{~L}$ & 108.00 \\
\hline $\mathrm{H} 38 \mathrm{~A}-\mathrm{C} 38-\mathrm{H} 38 \mathrm{C}$ & 109.00 & $\mathrm{C} 104-\mathrm{C} 103-\mathrm{H} 10 \mathrm{M}$ & 108.00 \\
\hline $\mathrm{C} 37-\mathrm{C} 38-\mathrm{H} 38 \mathrm{~A}$ & 109.00 & $\mathrm{H} 10 \mathrm{~L}-\mathrm{C} 103-\mathrm{H} 10 \mathrm{M}$ & 108.00 \\
\hline $\mathrm{C} 37-\mathrm{C} 38-\mathrm{H} 38 \mathrm{C}$ & 109.00 & $\mathrm{H} 10 \mathrm{~N}-\mathrm{C} 104-\mathrm{H} 10 \mathrm{O}$ & 109.00 \\
\hline $\mathrm{H} 38 \mathrm{~A}-\mathrm{C} 38-\mathrm{H} 38 \mathrm{~B}$ & 110.00 & $\mathrm{H} 10 \mathrm{~N}-\mathrm{C} 104-\mathrm{H} 10 \mathrm{P}$ & 109.00 \\
\hline $\mathrm{H} 38 \mathrm{~B}-\mathrm{C} 38-\mathrm{H} 38 \mathrm{C}$ & 109.00 & $\mathrm{H} 10 \mathrm{O}-\mathrm{C} 104-\mathrm{H} 10 \mathrm{P}$ & 110.00 \\
\hline $\mathrm{H} 39 \mathrm{~A}-\mathrm{C} 39-\mathrm{H} 39 \mathrm{C}$ & 109.00 & $\mathrm{C} 103-\mathrm{C} 104-\mathrm{H} 10 \mathrm{~N}$ & 109.00 \\
\hline $\mathrm{H} 39 \mathrm{~A}-\mathrm{C} 39-\mathrm{H} 39 \mathrm{~B}$ & 109.00 & $\mathrm{C} 103-\mathrm{C} 104-\mathrm{H} 10 \mathrm{O}$ & 109.00 \\
\hline C37-C39-H39C & 109.00 & $\mathrm{C} 103-\mathrm{C} 104-\mathrm{H} 10 \mathrm{P}$ & 110.00 \\
\hline C37- $339-\mathrm{H} 39 \mathrm{~A}$ & 109.00 & $\mathrm{C} 106-\mathrm{C} 105-\mathrm{H} 10 \mathrm{R}$ & 109.00 \\
\hline $\mathrm{H} 39 \mathrm{~B}-\mathrm{C} 39-\mathrm{H} 39 \mathrm{C}$ & 110.00 & $\mathrm{~N} 1-\mathrm{C} 105-\mathrm{H} 10 \mathrm{R}$ & 108.00 \\
\hline C37-C39-H39B & 109.00 & $\mathrm{~N} 1-\mathrm{C} 105-\mathrm{H} 10 \mathrm{~S}$ & 108.00 \\
\hline $\mathrm{C} 37-\mathrm{C} 40-\mathrm{H} 40 \mathrm{~A}$ & 109.00 & $\mathrm{C} 106-\mathrm{C} 105-\mathrm{H} 10 \mathrm{~S}$ & 108.00 \\
\hline $\mathrm{C} 37-\mathrm{C} 40-\mathrm{H} 40 \mathrm{C}$ & 109.00 & $\mathrm{H} 10 \mathrm{R}-\mathrm{C} 105-\mathrm{H} 10 \mathrm{~S}$ & 107.00 \\
\hline $\mathrm{H} 40 \mathrm{~A}-\mathrm{C} 40-\mathrm{H} 40 \mathrm{~B}$ & 110.00 & $\mathrm{C} 105-\mathrm{C} 106-\mathrm{H} 10 \mathrm{U}$ & 109.00 \\
\hline $\mathrm{C} 37-\mathrm{C} 40-\mathrm{H} 40 \mathrm{~B}$ & 109.00 & $\mathrm{C} 105-\mathrm{C} 106-\mathrm{H} 10 \mathrm{~T}$ & 110.00 \\
\hline $\mathrm{H} 40 \mathrm{~B}-\mathrm{C} 40-\mathrm{H} 40 \mathrm{C}$ & 110.00 & $\mathrm{H} 10 \mathrm{~T}-\mathrm{C} 106-\mathrm{H} 10 \mathrm{U}$ & 110.00 \\
\hline $\mathrm{H} 40 \mathrm{~A}-\mathrm{C} 40-\mathrm{H} 40 \mathrm{C}$ & 109.00 & $\mathrm{H} 10 \mathrm{~T}-\mathrm{C} 106-\mathrm{H} 10 \mathrm{~V}$ & 109.00 \\
\hline $\mathrm{C} 42-\mathrm{C} 41-\mathrm{H} 41 \mathrm{~A}$ & 110.00 & $\mathrm{C} 105-\mathrm{C} 106-\mathrm{H} 10 \mathrm{~V}$ & 109.00 \\
\hline $\mathrm{O} 4-\mathrm{C} 41-\mathrm{H} 41 \mathrm{~A}$ & 110.00 & $\mathrm{H} 10 \mathrm{U}-\mathrm{C} 106-\mathrm{H} 10 \mathrm{~V}$ & 110.00 \\
\hline $\mathrm{H} 41 \mathrm{~A}-\mathrm{C} 41-\mathrm{H} 41 \mathrm{~B}$ & 108.00 & $\mathrm{C} 108-\mathrm{C} 107-\mathrm{H} 10 \mathrm{Y}$ & 109.00 \\
\hline $\mathrm{C} 42-\mathrm{C} 41-\mathrm{H} 41 \mathrm{~B}$ & 110.00 & $\mathrm{C} 108-\mathrm{C} 107-\mathrm{H} 10 \mathrm{Z}$ & 109.00 \\
\hline $\mathrm{O} 4-\mathrm{C} 41-\mathrm{H} 41 \mathrm{~B}$ & 110.00 & $\mathrm{~N} 1-\mathrm{C} 107-\mathrm{H} 10 \mathrm{Y}$ & 109.00 \\
\hline $\mathrm{C} 43-\mathrm{C} 44-\mathrm{H} 44$ & 120.00 & $\mathrm{~N} 1-\mathrm{C} 107-\mathrm{H} 10 \mathrm{Z}$ & 109.00 \\
\hline $\mathrm{C} 45-\mathrm{C} 44-\mathrm{H} 44$ & 120.00 & $\mathrm{H} 10 \mathrm{Y}-\mathrm{C} 107-\mathrm{H} 10 \mathrm{Z}$ & 108.00 \\
\hline $\mathrm{C} 46-\mathrm{C} 45-\mathrm{H} 45$ & 120.00 & $\mathrm{H} 10 \mathrm{Q}-\mathrm{C} 108-\mathrm{H} 10 \mathrm{~W}$ & 109.00 \\
\hline $\mathrm{C} 44-\mathrm{C} 45-\mathrm{H} 45$ & 120.00 & $\mathrm{C} 107-\mathrm{C} 108-\mathrm{H} 1 \mathrm{QW}$ & 109.00 \\
\hline $\mathrm{C} 45-\mathrm{C} 46-\mathrm{H} 46$ & 120.00 & C107-C108-H10Q & 109.00 \\
\hline $\mathrm{C} 47-\mathrm{C} 46-\mathrm{H} 46$ & 120.00 & $\mathrm{C} 107-\mathrm{C} 108-\mathrm{H} 10 \mathrm{~W}$ & 109.00 \\
\hline $\mathrm{C} 46-\mathrm{C} 47-\mathrm{H} 47$ & 120.00 & $\mathrm{H} 1 \mathrm{QW}-\mathrm{C} 108-\mathrm{H} 10 \mathrm{Q}$ & 110.00 \\
\hline $\mathrm{C} 48-\mathrm{C} 47-\mathrm{H} 47$ & 120.00 & $\mathrm{H} 1 \mathrm{QW}-\mathrm{C} 108-\mathrm{H} 10 \mathrm{~W}$ & 109.00 \\
\hline $\mathrm{C} 47-\mathrm{C} 48-\mathrm{H} 48$ & 120.00 & $\mathrm{~N} 2-\mathrm{C} 201-\mathrm{C} 202$ & $116.6(8)$ \\
\hline $\mathrm{C} 43-\mathrm{C} 48-\mathrm{H} 48$ & 120.00 & $\mathrm{~N} 2-\mathrm{C} 203-\mathrm{C} 204$ & $115.9(8)$ \\
\hline $\mathrm{C} 2^{\prime}-\mathrm{C} 1^{\prime}-\mathrm{C} 6^{\prime}$ & $121.4(5)$ & $\mathrm{N} 2-\mathrm{C} 205-\mathrm{C} 206$ & $113.2(9)$ \\
\hline $\mathrm{C} 1^{\prime}-\mathrm{C} 2^{\prime}-\mathrm{C} 3^{\prime}$ & $120.9(5)$ & $\mathrm{N} 2-\mathrm{C} 207-\mathrm{C} 208$ & $115.5(10)$ \\
\hline $\mathrm{S} 1^{\prime}-\mathrm{C} 2^{\prime}-\mathrm{C} 3^{\prime}$ & $119.9(4)$ & $\mathrm{N} 2-\mathrm{C} 201-\mathrm{H} 20 \mathrm{G}$ & 108.00 \\
\hline $\mathrm{S} 1^{\prime}-\mathrm{C} 2^{\prime}-\mathrm{C} 1^{\prime}$ & $119.2(4)$ & $\mathrm{N} 2-\mathrm{C} 201-\mathrm{H} 20 \mathrm{H}$ & 108.00 \\
\hline $\mathrm{O} 1^{\prime}-\mathrm{C} 3^{\prime}-\mathrm{C} 2^{\prime}$ & $118.2(5)$ & $\mathrm{C} 202-\mathrm{C} 201-\mathrm{H} 20 \mathrm{G}$ & 108.00 \\
\hline $\mathrm{O} 1^{\prime}-\mathrm{C} 3^{\prime}-\mathrm{C}^{\prime}$ & $124.3(5)$ & $\mathrm{C} 202-\mathrm{C} 201-\mathrm{H} 20 \mathrm{H}$ & 108.00 \\
\hline $\mathrm{C} 2^{\prime}-\mathrm{C} 3^{\prime}-\mathrm{C} 4^{\prime}$ & $117.5(5)$ & $\mathrm{H} 20 \mathrm{G}-\mathrm{C} 201-\mathrm{H} 20 \mathrm{H}$ & 107.00 \\
\hline $\mathrm{C} 3^{\prime}-\mathrm{C} 4^{\prime}-\mathrm{C} 5^{\prime}$ & $120.4(5)$ & C201-C202-H20I & 109.00 \\
\hline $\mathrm{S} 2^{\prime}-\mathrm{C} 4^{\prime}-\mathrm{C} 3^{\prime}$ & $121.0(4)$ & $\mathrm{C} 201-\mathrm{C} 202-\mathrm{H} 20 \mathrm{~J}$ & 109.00 \\
\hline $\mathrm{S} 2^{\prime}-\mathrm{C} 4^{\prime}-\mathrm{C} 5^{\prime}$ & $118.3(4)$ & $\mathrm{C} 201-\mathrm{C} 202-\mathrm{H} 20 \mathrm{~K}$ & 110.00 \\
\hline
\end{tabular}




\begin{tabular}{|c|c|}
\hline $\mathrm{C} 4^{\prime}-\mathrm{C} 5^{\prime}-\mathrm{C} 6^{\prime}$ & $121.8(5)$ \\
\hline $\mathrm{C} 1^{\prime}-\mathrm{C} 6^{\prime}-\mathrm{C} 5^{\prime}$ & $117.8(6)$ \\
\hline $\mathrm{C} 1^{\prime}-\mathrm{C} 6^{\prime}-\mathrm{C} 7^{\prime}$ & $122.6(5)$ \\
\hline $\mathrm{C} 5^{\prime}-\mathrm{C} 6^{\prime}-\mathrm{C} 7^{\prime}$ & $119.5(5)$ \\
\hline $\mathrm{C} 8^{\prime}-\mathrm{C} 7^{\prime}-\mathrm{C} 9^{\prime}$ & $112.8(8)$ \\
\hline $\mathrm{C} 8^{\prime}-\mathrm{C} 7^{\prime}-\mathrm{C} 10^{\prime}$ & $106.5(8)$ \\
\hline $\mathrm{C} 9^{\prime}-\mathrm{C} 7^{\prime}-\mathrm{C} 10^{\prime}$ & $107.1(7)$ \\
\hline $\mathrm{C} 6^{\prime}-\mathrm{C} 7^{\prime}-\mathrm{C} 9^{\prime}$ & $108.0(6)$ \\
\hline $\mathrm{C} 6^{\prime}-\mathrm{C} 7^{\prime}-\mathrm{C} 10^{\prime}$ & $111.1(7)$ \\
\hline $\mathrm{C} 6^{\prime}-\mathrm{C} 7^{\prime}-\mathrm{C} 8^{\prime}$ & $111.3(7)$ \\
\hline $\mathrm{C} 12^{\prime}-\mathrm{C} 11^{\prime}-\mathrm{C} 16^{\prime}$ & $122.1(6)$ \\
\hline $\mathrm{S} 2^{\prime}-\mathrm{C} 12^{\prime}-\mathrm{C} 11^{\prime}$ & $119.0(4)$ \\
\hline $\mathrm{S} 2^{\prime}-\mathrm{C} 12^{\prime}-\mathrm{C} 13^{\prime}$ & $119.2(3)$ \\
\hline $\mathrm{C} 11^{\prime}-\mathrm{C} 12^{\prime}-\mathrm{C} 13^{\prime}$ & $121.4(5)$ \\
\hline $\mathrm{O} 2^{\prime}-\mathrm{C} 13^{\prime}-\mathrm{C} 14^{\prime}$ & $122.3(5)$ \\
\hline $\mathrm{O} 2^{\prime}-\mathrm{C} 13^{\prime}-\mathrm{C} 12^{\prime}$ & $122.2(5)$ \\
\hline $\mathrm{C} 12^{\prime}-\mathrm{C} 13^{\prime}-\mathrm{C} 14^{\prime}$ & $115.6(4)$ \\
\hline $\mathrm{S} 33^{\prime}-\mathrm{C} 14^{\prime}-\mathrm{C} 13^{\prime}$ & $119.5(3)$ \\
\hline $\mathrm{S} 3^{\prime}-\mathrm{C} 14^{\prime}-\mathrm{C} 15^{\prime}$ & $119.6(4)$ \\
\hline $\mathrm{C} 13^{\prime}-\mathrm{C} 14^{\prime}-\mathrm{C} 15^{\prime}$ & $120.9(4)$ \\
\hline $\mathrm{C} 14^{\prime}-\mathrm{C} 15^{\prime}-\mathrm{C} 16^{\prime}$ & $123.7(5)$ \\
\hline $\mathrm{C} 11^{\prime}-\mathrm{C} 16^{\prime}-\mathrm{C} 15^{\prime}$ & $115.6(5)$ \\
\hline $\mathrm{C} 15^{\prime}-\mathrm{C} 16^{\prime}-\mathrm{C} 17^{\prime}$ & $122.3(6)$ \\
\hline $\mathrm{C} 11^{\prime}-\mathrm{C} 16^{\prime}-\mathrm{C} 17^{\prime}$ & $121.9(6)$ \\
\hline $\mathrm{C} 16^{\prime}-\mathrm{C} 17^{\prime}-\mathrm{C} 19^{\prime}$ & $108.3(6)$ \\
\hline $\mathrm{C} 18^{\prime}-\mathrm{C} 17^{\prime}-\mathrm{C} 20^{\prime}$ & $106.0(7)$ \\
\hline $\mathrm{C} 16^{\prime}-\mathrm{C} 17^{\prime}-\mathrm{C} 20^{\prime}$ & $108.9(6)$ \\
\hline $\mathrm{C} 18^{\prime}-\mathrm{C} 17^{\prime}-\mathrm{C} 19^{\prime}$ & $108.3(10)$ \\
\hline $\mathrm{C} 16^{\prime}-\mathrm{C} 17^{\prime}-\mathrm{C} 18^{\prime}$ & $112.4(6)$ \\
\hline $\mathrm{C} 19^{\prime}-\mathrm{C} 17^{\prime}-\mathrm{C} 20^{\prime}$ & $113.0(10)$ \\
\hline $\mathrm{C} 22^{\prime}-\mathrm{C} 21^{\prime}-\mathrm{C} 26^{\prime}$ & $122.6(5)$ \\
\hline $\mathrm{S} 33^{\prime}-\mathrm{C} 22^{\prime}-\mathrm{C} 23^{\prime}$ & $120.8(4)$ \\
\hline $\mathrm{S} 3^{\prime}-\mathrm{C} 22^{\prime}-\mathrm{C} 21^{\prime}$ & $118.4(4)$ \\
\hline $\mathrm{C} 21^{\prime}-\mathrm{C} 22^{\prime}-\mathrm{C} 23^{\prime}$ & $120.7(5)$ \\
\hline $\mathrm{O} 3^{\prime}-\mathrm{C} 23^{\prime}-\mathrm{C} 22^{\prime}$ & $123.9(5)$ \\
\hline $\mathrm{O}^{\prime}-\mathrm{C} 23^{\prime}-\mathrm{C} 24^{\prime}$ & $118.7(5)$ \\
\hline $\mathrm{C} 2-\mathrm{S} 1-\mathrm{C} 34-\mathrm{C} 33$ & $-132.0(5)$ \\
\hline $\mathrm{C} 2-\mathrm{S} 1-\mathrm{C} 34-\mathrm{C} 35$ & $42.1(5)$ \\
\hline $\mathrm{C} 34-\mathrm{S} 1-\mathrm{C} 2-\mathrm{C} 3$ & $59.3(5)$ \\
\hline $\mathrm{C} 34-\mathrm{S} 1-\mathrm{C} 2-\mathrm{C} 1$ & $-119.7(5)$ \\
\hline $\mathrm{C} 4-\mathrm{S} 2-\mathrm{C} 12-\mathrm{C} 11$ & $-90.9(5)$ \\
\hline $\mathrm{C} 4-\mathrm{S} 2-\mathrm{C} 12-\mathrm{C} 13$ & $95.8(4)$ \\
\hline $\mathrm{C} 12-\mathrm{S} 2-\mathrm{C} 4-\mathrm{C} 3$ & $-70.4(4)$ \\
\hline $\mathrm{C} 12-\mathrm{S} 2-\mathrm{C} 4-\mathrm{C} 5$ & $116.5(4)$ \\
\hline $\mathrm{C} 22-\mathrm{S} 3-\mathrm{C} 14-\mathrm{C} 15$ & $89.6(4)$ \\
\hline $\mathrm{C} 14-\mathrm{S} 3-\mathrm{C} 22-\mathrm{C} 21$ & $-106.3(5)$ \\
\hline $\mathrm{C} 22-\mathrm{S} 3-\mathrm{C} 14-\mathrm{C} 13$ & $-93.6(4)$ \\
\hline
\end{tabular}

\begin{tabular}{|c|c|}
\hline $\mathrm{H} 20 \mathrm{I}-\mathrm{C} 202-\mathrm{H} 20 \mathrm{~J}$ & 109.00 \\
\hline $\mathrm{H} 20 \mathrm{I}-\mathrm{C} 202-\mathrm{H} 20 \mathrm{~K}$ & 109.00 \\
\hline $\mathrm{H} 20 \mathrm{~J}-\mathrm{C} 202-\mathrm{H} 20 \mathrm{~K}$ & 110.00 \\
\hline $\mathrm{N} 2-\mathrm{C} 203-\mathrm{H} 20 \mathrm{~L}$ & 108.00 \\
\hline $\mathrm{N} 2-\mathrm{C} 203-\mathrm{H} 20 \mathrm{M}$ & 108.00 \\
\hline $\mathrm{C} 204-\mathrm{C} 203-\mathrm{H} 20 \mathrm{~L}$ & 108.00 \\
\hline $\mathrm{C} 204-\mathrm{C} 203-\mathrm{H} 20 \mathrm{M}$ & 108.00 \\
\hline $\mathrm{H} 20 \mathrm{~L}-\mathrm{C} 203-\mathrm{H} 20 \mathrm{M}$ & 107.00 \\
\hline $\mathrm{C} 203-\mathrm{C} 204-\mathrm{H} 20 \mathrm{~N}$ & 109.00 \\
\hline $\mathrm{C} 203-\mathrm{C} 204-\mathrm{H} 20 \mathrm{O}$ & 110.00 \\
\hline $\mathrm{C} 203-\mathrm{C} 204-\mathrm{H} 20 \mathrm{P}$ & 110.00 \\
\hline $\mathrm{H} 20 \mathrm{~N}-\mathrm{C} 204-\mathrm{H} 20 \mathrm{O}$ & 109.00 \\
\hline $\mathrm{H} 20 \mathrm{~N}-\mathrm{C} 204-\mathrm{H} 20 \mathrm{P}$ & 109.00 \\
\hline $\mathrm{H} 20 \mathrm{O}-\mathrm{C} 204-\mathrm{H} 20 \mathrm{P}$ & 109.00 \\
\hline $\mathrm{N} 2-\mathrm{C} 205-\mathrm{H} 20 \mathrm{R}$ & 109.00 \\
\hline $\mathrm{N} 2-\mathrm{C} 205-\mathrm{H} 20 \mathrm{~S}$ & 109.00 \\
\hline $\mathrm{C} 206-\mathrm{C} 205-\mathrm{H} 20 \mathrm{R}$ & 109.00 \\
\hline $\mathrm{C} 206-\mathrm{C} 205-\mathrm{H} 20 \mathrm{~S}$ & 109.00 \\
\hline $\mathrm{H} 20 \mathrm{R}-\mathrm{C} 205-\mathrm{H} 20 \mathrm{~S}$ & 108.00 \\
\hline $\mathrm{C} 205-\mathrm{C} 206-\mathrm{H} 20 \mathrm{~T}$ & 110.00 \\
\hline $\mathrm{C} 205-\mathrm{C} 206-\mathrm{H} 20 \mathrm{U}$ & 110.00 \\
\hline $\mathrm{C} 205-\mathrm{C} 206-\mathrm{H} 20 \mathrm{~V}$ & 109.00 \\
\hline $\mathrm{H} 20 \mathrm{~T}-\mathrm{C} 206-\mathrm{H} 20 \mathrm{U}$ & 109.00 \\
\hline $\mathrm{H} 20 \mathrm{~T}-\mathrm{C} 206-\mathrm{H} 20 \mathrm{~V}$ & 109.00 \\
\hline $\mathrm{H} 20 \mathrm{U}-\mathrm{C} 206-\mathrm{H} 20 \mathrm{~V}$ & 109.00 \\
\hline $\mathrm{N} 2-\mathrm{C} 207-\mathrm{H} 20 \mathrm{Y}$ & 109.00 \\
\hline $\mathrm{N} 2-\mathrm{C} 207-\mathrm{H} 20 \mathrm{Z}$ & 108.00 \\
\hline C208-C207-H20Y & 108.00 \\
\hline $\mathrm{C} 208-\mathrm{C} 207-\mathrm{H} 20 \mathrm{Z}$ & 108.00 \\
\hline $\mathrm{H} 20 \mathrm{Y}-\mathrm{C} 207-\mathrm{H} 20 \mathrm{Z}$ & 107.00 \\
\hline $\mathrm{C} 207-\mathrm{C} 208-\mathrm{H} 2 \mathrm{QW}$ & 109.00 \\
\hline $\mathrm{C} 207-\mathrm{C} 208-\mathrm{H} 20 \mathrm{Q}$ & 109.00 \\
\hline $\mathrm{C} 207-\mathrm{C} 208-\mathrm{H} 20 \mathrm{~W}$ & 109.00 \\
\hline $\mathrm{H} 2 \mathrm{QW}-\mathrm{C} 208-\mathrm{H} 20 \mathrm{Q}$ & 109.00 \\
\hline $\mathrm{H} 2 \mathrm{QW}-\mathrm{C} 208-\mathrm{H} 20 \mathrm{~W}$ & 110.00 \\
\hline $\mathrm{H} 20 \mathrm{Q}-\mathrm{C} 208-\mathrm{H} 20 \mathrm{~W}$ & 109.00 \\
\hline $\mathrm{C} 42-\mathrm{C} 43-\mathrm{C} 48-\mathrm{C} 47$ & $179.9(6)$ \\
\hline $\mathrm{C} 42-\mathrm{C} 43-\mathrm{C} 44-\mathrm{C} 45$ & $-179.5(6)$ \\
\hline $\mathrm{C} 48-\mathrm{C} 43-\mathrm{C} 44-\mathrm{C} 45$ & $0.7(9)$ \\
\hline $\mathrm{C} 43-\mathrm{C} 44-\mathrm{C} 45-\mathrm{C} 46$ & $-1.1(11)$ \\
\hline $\mathrm{C} 44-\mathrm{C} 45-\mathrm{C} 46-\mathrm{C} 47$ & $1.1(12)$ \\
\hline $\mathrm{C} 45-\mathrm{C} 46-\mathrm{C} 47-\mathrm{C} 48$ & $-0.6(12)$ \\
\hline $\mathrm{C} 46-\mathrm{C} 47-\mathrm{C} 48-\mathrm{C} 43$ & $0.3(11)$ \\
\hline $\mathrm{C} 2^{\prime}-\mathrm{C} 1^{\prime}-\mathrm{C} 6^{\prime}-\mathrm{C} 7^{\prime}$ & $178.7(6)$ \\
\hline $\mathrm{C} 6^{\prime}-\mathrm{C} 1^{\prime}-\mathrm{C} 2^{\prime}-\mathrm{C} 3^{\prime}$ & $-1.3(9)$ \\
\hline $\mathrm{C} 2^{\prime}-\mathrm{C} 1^{\prime}-\mathrm{C} 6^{\prime}-\mathrm{C} 5^{\prime}$ & $-0.3(9)$ \\
\hline $\mathrm{C} 6^{\prime}-\mathrm{C} 1^{\prime}-\mathrm{C} 2^{\prime}-\mathrm{S} 1^{\prime}$ & $177.4(5)$ \\
\hline
\end{tabular}




\begin{tabular}{|c|c|}
\hline $\mathrm{C} 14-\mathrm{S} 3-\mathrm{C} 22-\mathrm{C} 23$ & $76.4(4)$ \\
\hline $\mathrm{C} 32-\mathrm{S} 4-\mathrm{C} 24-\mathrm{C} 25$ & $109.1(4)$ \\
\hline $\mathrm{C} 32-\mathrm{S} 4-\mathrm{C} 24-\mathrm{C} 23$ & $-67.1(4)$ \\
\hline $\mathrm{C} 24-\mathrm{S} 4-\mathrm{C} 32-\mathrm{C} 31$ & $-39.0(4)$ \\
\hline $\mathrm{C} 24-\mathrm{S} 4-\mathrm{C} 32-\mathrm{C} 33$ & $135.1(4)$ \\
\hline $\mathrm{C} 34^{\prime}-\mathrm{S} 1^{\prime}-\mathrm{C} 2^{\prime}-\mathrm{C} 1^{\prime}$ & $-118.2(5)$ \\
\hline $\mathrm{C} 34^{\prime}-\mathrm{S} 1^{\prime}-\mathrm{C} 2^{\prime}-\mathrm{C} 3^{\prime}$ & $60.5(5)$ \\
\hline $\mathrm{C} 2^{\prime}-\mathrm{S} 1^{\prime}-\mathrm{C} 34^{\prime}-\mathrm{C} 35^{\prime}$ & $41.9(5)$ \\
\hline $\mathrm{C} 2^{\prime}-\mathrm{S} 1^{\prime}-\mathrm{C} 34^{\prime}-\mathrm{C} 33^{\prime}$ & $-132.0(5)$ \\
\hline $\mathrm{C} 12^{\prime}-\mathrm{S} 2^{\prime}-\mathrm{C} 4^{\prime}-\mathrm{C} 3^{\prime}$ & $-70.9(5)$ \\
\hline $\mathrm{C} 4^{\prime}-\mathrm{S} 2^{\prime}-\mathrm{C} 12^{\prime}-\mathrm{C} 11^{\prime}$ & $-90.7(5)$ \\
\hline $\mathrm{C} 4^{\prime}-\mathrm{S} 2^{\prime}-\mathrm{C} 12^{\prime}-\mathrm{C} 13^{\prime}$ & $96.3(5)$ \\
\hline $\mathrm{C} 12^{\prime}-\mathrm{S} 2^{\prime}-\mathrm{C} 4^{\prime}-\mathrm{C} 5^{\prime}$ & $114.7(5)$ \\
\hline $\mathrm{C} 14^{\prime}-\mathrm{S} 3^{\prime}-\mathrm{C} 22^{\prime}-\mathrm{C} 21^{\prime}$ & $-106.6(4)$ \\
\hline $\mathrm{C} 14^{\prime}-\mathrm{S} 33^{\prime}-\mathrm{C} 22^{\prime}-\mathrm{C} 23^{\prime}$ & $77.0(4)$ \\
\hline $\mathrm{C} 22^{\prime}-\mathrm{S} 3^{\prime}-\mathrm{C} 14^{\prime}-\mathrm{C} 15^{\prime}$ & $90.0(5)$ \\
\hline $\mathrm{C} 22^{\prime}-\mathrm{S} 3^{\prime}-\mathrm{C} 14^{\prime}-\mathrm{C} 13^{\prime}$ & $-93.6(4)$ \\
\hline $\mathrm{C} 32^{\prime}-\mathrm{S} 4^{\prime}-\mathrm{C} 24^{\prime}-\mathrm{C} 25^{\prime}$ & $109.3(4)$ \\
\hline $\mathrm{C} 24^{\prime}-\mathrm{S} 44^{\prime}-\mathrm{C} 32^{\prime}-\mathrm{C} 31^{\prime}$ & $-39.8(4)$ \\
\hline $\mathrm{C} 32^{\prime}-\mathrm{S} 4^{\prime}-\mathrm{C} 24^{\prime}-\mathrm{C} 23^{\prime}$ & $-66.6(4)$ \\
\hline $\mathrm{C} 24^{\prime}-\mathrm{S} 4^{\prime}-\mathrm{C} 32^{\prime}-\mathrm{C} 33^{\prime}$ & $134.6(4)$ \\
\hline $\mathrm{C} 41-\mathrm{O} 4-\mathrm{C} 33-\mathrm{C} 34$ & $91.7(5)$ \\
\hline $\mathrm{C} 41-\mathrm{O} 4-\mathrm{C} 33-\mathrm{C} 32$ & $-88.4(5)$ \\
\hline $\mathrm{C} 33-\mathrm{O} 4-\mathrm{C} 41-\mathrm{C} 42$ & $177.2(5)$ \\
\hline $\mathrm{C} 6-\mathrm{C} 1-\mathrm{C} 2-\mathrm{C} 3$ & $-1.1(9)$ \\
\hline $\mathrm{C} 2-\mathrm{C} 1-\mathrm{C} 6-\mathrm{C} 5$ & $-1.2(9)$ \\
\hline $\mathrm{C} 2-\mathrm{C} 1-\mathrm{C} 6-\mathrm{C} 7$ & $179.3(6)$ \\
\hline $\mathrm{C} 6-\mathrm{C} 1-\mathrm{C} 2-\mathrm{S} 1$ & $177.8(5)$ \\
\hline $\mathrm{C} 1-\mathrm{C} 2-\mathrm{C} 3-\mathrm{C} 4$ & $3.5(8)$ \\
\hline $\mathrm{S} 1-\mathrm{C} 2-\mathrm{C} 3-\mathrm{C} 4$ & $-175.5(4)$ \\
\hline $\mathrm{C} 1-\mathrm{C} 2-\mathrm{C} 3-\mathrm{O} 1$ & $-176.5(5)$ \\
\hline $\mathrm{S} 1-\mathrm{C} 2-\mathrm{C} 3-\mathrm{O} 1$ & $4.6(7)$ \\
\hline $\mathrm{C} 2-\mathrm{C} 3-\mathrm{C} 4-\mathrm{S} 2$ & $-176.4(4)$ \\
\hline $\mathrm{C} 2-\mathrm{C} 3-\mathrm{C} 4-\mathrm{C} 5$ & $-3.5(7)$ \\
\hline $\mathrm{O} 1-\mathrm{C} 3-\mathrm{C} 4-\mathrm{S} 2$ & $3.6(7)$ \\
\hline $\mathrm{O} 1-\mathrm{C} 3-\mathrm{C} 4-\mathrm{C} 5$ & $176.5(5)$ \\
\hline $\mathrm{C} 3-\mathrm{C} 4-\mathrm{C} 5-\mathrm{C} 6$ & $1.3(8)$ \\
\hline $\mathrm{S} 2-\mathrm{C} 4-\mathrm{C} 5-\mathrm{C} 6$ & $174.3(5)$ \\
\hline $\mathrm{C} 41^{\prime}-\mathrm{O} 4^{\prime}-\mathrm{C} 33^{\prime}-\mathrm{C} 34^{\prime}$ & $93.0(5)$ \\
\hline $\mathrm{C} 41^{\prime}-\mathrm{O} 4^{\prime}-\mathrm{C} 33^{\prime}-\mathrm{C} 32^{\prime}$ & $-87.6(5)$ \\
\hline $\mathrm{C} 33^{\prime}-\mathrm{O} 4^{\prime}-\mathrm{C} 41^{\prime}-\mathrm{C} 42^{\prime}$ & $176.7(4)$ \\
\hline $\mathrm{C} 4-\mathrm{C} 5-\mathrm{C} 6-\mathrm{C} 7$ & $-179.4(5)$ \\
\hline $\mathrm{C} 4-\mathrm{C} 5-\mathrm{C} 6-\mathrm{C} 1$ & $1.2(9)$ \\
\hline $\mathrm{C} 1-\mathrm{C} 6-\mathrm{C} 7-\mathrm{C} 8$ & $5.1(10)$ \\
\hline $\mathrm{C} 5-\mathrm{C} 6-\mathrm{C} 7-\mathrm{C} 8$ & $-174.4(7)$ \\
\hline $\mathrm{C} 1-\mathrm{C} 6-\mathrm{C} 7-\mathrm{C} 10$ & $125.9(8)$ \\
\hline $\mathrm{C} 5-\mathrm{C} 6-\mathrm{C} 7-\mathrm{C} 10$ & $-53.5(9)$ \\
\hline $\mathrm{C} 1-\mathrm{C} 6-\mathrm{C} 7-\mathrm{C} 9$ & $-116.3(7)$ \\
\hline
\end{tabular}

\begin{tabular}{|c|c|}
\hline $\mathrm{C} 1^{\prime}-\mathrm{C} 2^{\prime}-\mathrm{C} 3^{\prime}-\mathrm{O}^{\prime}$ & $-177.0(5)$ \\
\hline $\mathrm{S} 1^{\prime}-\mathrm{C} 2^{\prime}-\mathrm{C} 3^{\prime}-\mathrm{C} 4^{\prime}$ & $-176.2(4)$ \\
\hline $\mathrm{C} 1^{\prime}-\mathrm{C} 2^{\prime}-\mathrm{C} 3^{\prime}-\mathrm{C} 4^{\prime}$ & $2.5(8)$ \\
\hline $\mathrm{S} 1^{\prime}-\mathrm{C} 2^{\prime}-\mathrm{C}^{\prime}-\mathrm{O}^{\prime}$ & $4.3(7)$ \\
\hline $\mathrm{C} 2^{\prime}-\mathrm{C} 3^{\prime}-\mathrm{C} 4^{\prime}-\mathrm{C} 5^{\prime}$ & $-2.3(8)$ \\
\hline $\mathrm{O} 1^{\prime}-\mathrm{C} 3^{\prime}-\mathrm{C} 4^{\prime}-\mathrm{C} 5^{\prime}$ & $177.2(5)$ \\
\hline $\mathrm{C} 2^{\prime}-\mathrm{C} 3^{\prime}-\mathrm{C} 4^{\prime}-\mathrm{S} 2^{\prime}$ & $-176.5(4)$ \\
\hline $\mathrm{O} 1^{\prime}-\mathrm{C} 3^{\prime}-\mathrm{C} 4^{\prime}-\mathrm{S} 2^{\prime}$ & $2.9(8)$ \\
\hline $\mathrm{C} 3^{\prime}-\mathrm{C} 4^{\prime}-\mathrm{C} 5^{\prime}-\mathrm{C} 6^{\prime}$ & $0.8(9)$ \\
\hline $\mathrm{S} 2^{\prime}-\mathrm{C} 4^{\prime}-\mathrm{C} 5^{\prime}-\mathrm{C} 6^{\prime}$ & $175.2(5)$ \\
\hline $\mathrm{C} 4^{\prime}-\mathrm{C} 5^{\prime}-\mathrm{C} 6^{\prime}-\mathrm{C} 7^{\prime}$ & $-178.5(6)$ \\
\hline $\mathrm{C}^{\prime}-\mathrm{C} 5^{\prime}-\mathrm{C} 6^{\prime}-\mathrm{C}^{\prime}$ & $0.5(9)$ \\
\hline $\mathrm{C} 1^{\prime}-\mathrm{C} 6^{\prime}-\mathrm{C} 7^{\prime}-\mathrm{C} 10^{\prime}$ & $133.5(7)$ \\
\hline $\mathrm{C} 5^{\prime}-\mathrm{C} 6^{\prime}-\mathrm{C} 7^{\prime}-\mathrm{C} 8^{\prime}$ & $-166.0(7)$ \\
\hline $\mathrm{C} 5^{\prime}-\mathrm{C} 6^{\prime}-\mathrm{C} 7^{\prime}-\mathrm{C} 9^{\prime}$ & $69.7(8)$ \\
\hline $\mathrm{C} 1^{\prime}-\mathrm{C} 6^{\prime}-\mathrm{C} 7^{\prime}-\mathrm{C} 9^{\prime}$ & $-109.3(7)$ \\
\hline $\mathrm{C} 5^{\prime}-\mathrm{C} 6^{\prime}-\mathrm{C} 7^{\prime}-\mathrm{C} 10^{\prime}$ & $-47.5(9)$ \\
\hline $\mathrm{C} 1^{\prime}-\mathrm{C} 6^{\prime}-\mathrm{C} 7^{\prime}-\mathrm{C} 8^{\prime}$ & $15.1(10)$ \\
\hline $\mathrm{C} 16^{\prime}-\mathrm{C} 11^{\prime}-\mathrm{C} 12^{\prime}-\mathrm{S} 2^{\prime}$ & $-171.1(5)$ \\
\hline $\mathrm{C} 12^{\prime}-\mathrm{C} 11^{\prime}-\mathrm{C} 16^{\prime}-\mathrm{C} 15^{\prime}$ & $5.1(8)$ \\
\hline $\mathrm{C} 12^{\prime}-\mathrm{C} 11^{\prime}-\mathrm{C} 16^{\prime}-\mathrm{C} 17^{\prime}$ & $-178.4(6)$ \\
\hline $\mathrm{C} 16^{\prime}-\mathrm{C} 11^{\prime}-\mathrm{C} 12^{\prime}-\mathrm{C} 13^{\prime}$ & $1.8(9)$ \\
\hline $\mathrm{C} 11^{\prime}-\mathrm{C} 12^{\prime}-\mathrm{C} 13^{\prime}-\mathrm{O} 2^{\prime}$ & $170.8(5)$ \\
\hline $\mathrm{C} 11^{\prime}-\mathrm{C} 12^{\prime}-\mathrm{C} 13^{\prime}-\mathrm{C} 14^{\prime}$ & $-8.3(7)$ \\
\hline $\mathrm{S} 2^{\prime}-\mathrm{C} 12^{\prime}-\mathrm{C} 13^{\prime}-\mathrm{O} 2^{\prime}$ & $-16.4(6)$ \\
\hline $\mathrm{S} 2^{\prime}-\mathrm{C} 12^{\prime}-\mathrm{C} 13^{\prime}-\mathrm{C} 14^{\prime}$ & $164.5(4)$ \\
\hline $\mathrm{O} 2^{\prime}-\mathrm{C} 13^{\prime}-\mathrm{C} 14^{\prime}-\mathrm{S} 3^{\prime}$ & $12.7(7)$ \\
\hline $\mathrm{O} 2^{\prime}-\mathrm{C} 13^{\prime}-\mathrm{C} 14^{\prime}-\mathrm{C} 15^{\prime}$ & $-171.0(5)$ \\
\hline $\mathrm{C} 12^{\prime}-\mathrm{C} 13^{\prime}-\mathrm{C} 14^{\prime}-\mathrm{C} 15^{\prime}$ & $8.1(7)$ \\
\hline $\mathrm{C} 12^{\prime}-\mathrm{C} 13^{\prime}-\mathrm{C} 14^{\prime}-\mathrm{S} 3^{\prime}$ & $-168.2(4)$ \\
\hline $\mathrm{S} 3^{\prime}-\mathrm{C} 14^{\prime}-\mathrm{C} 15^{\prime}-\mathrm{C} 16^{\prime}$ & $174.9(5)$ \\
\hline $\mathrm{C} 13^{\prime}-\mathrm{C} 14^{\prime}-\mathrm{C} 15^{\prime}-\mathrm{C} 16^{\prime}$ & $-1.5(9)$ \\
\hline $\mathrm{C} 14^{\prime}-\mathrm{C} 15^{\prime}-\mathrm{C} 16^{\prime}-\mathrm{C} 17^{\prime}$ & $178.2(6)$ \\
\hline $\mathrm{C} 14^{\prime}-\mathrm{C} 15^{\prime}-\mathrm{C} 16^{\prime}-\mathrm{C} 11^{\prime}$ & $-5.3(9)$ \\
\hline $\mathrm{C} 15^{\prime}-\mathrm{C} 16^{\prime}-\mathrm{C} 17^{\prime}-\mathrm{C} 20^{\prime}$ & $-54.5(9)$ \\
\hline $\mathrm{C} 15^{\prime}-\mathrm{C} 16^{\prime}-\mathrm{C} 17^{\prime}-\mathrm{C} 18^{\prime}$ & $-171.7(8)$ \\
\hline $\mathrm{C} 11^{\prime}-\mathrm{C} 16^{\prime}-\mathrm{C} 17^{\prime}-\mathrm{C} 19^{\prime}$ & $-107.6(10$ \\
\hline $\mathrm{C} 15^{\prime}-\mathrm{C} 16^{\prime}-\mathrm{C} 17^{\prime}-\mathrm{C} 19^{\prime}$ & $68.7(11)$ \\
\hline $\mathrm{C} 11^{\prime}-\mathrm{C} 16^{\prime}-\mathrm{C} 17^{\prime}-\mathrm{C} 18^{\prime}$ & $12.0(10)$ \\
\hline $\mathrm{C} 11^{\prime}-\mathrm{C} 16^{\prime}-\mathrm{C} 17^{\prime}-\mathrm{C} 20^{\prime}$ & $129.2(8)$ \\
\hline $\mathrm{C} 22^{\prime}-\mathrm{C} 21^{\prime}-\mathrm{C} 26^{\prime}-\mathrm{C} 27^{\prime}$ & $-177.3(5)$ \\
\hline $\mathrm{C} 26^{\prime}-\mathrm{C} 21^{\prime}-\mathrm{C} 22^{\prime}-\mathrm{S} 3^{\prime}$ & $-178.3(5)$ \\
\hline $\mathrm{C} 22^{\prime}-\mathrm{C} 21^{\prime}-\mathrm{C} 26^{\prime}-\mathrm{C} 25^{\prime}$ & $1.7(9)$ \\
\hline $\mathrm{C} 26^{\prime}-\mathrm{C} 21^{\prime}-\mathrm{C} 22^{\prime}-\mathrm{C} 23^{\prime}$ & $-1.8(9)$ \\
\hline $\mathrm{S} 3^{\prime}-\mathrm{C} 22^{\prime}-\mathrm{C} 23^{\prime}-\mathrm{O}^{\prime}$ & $-2.2(7)$ \\
\hline $\mathrm{C} 21^{\prime}-\mathrm{C} 22^{\prime}-\mathrm{C} 23^{\prime}-\mathrm{C} 24^{\prime}$ & $1.7(7)$ \\
\hline $\mathrm{S} 3^{\prime}-\mathrm{C} 22^{\prime}-\mathrm{C} 23^{\prime}-\mathrm{C} 24^{\prime}$ & $178.1(4)$ \\
\hline $\mathrm{C} 21^{\prime}-\mathrm{C} 22^{\prime}-\mathrm{C} 23^{\prime}-\mathrm{O}^{\prime}$ & $-178.6(5)$ \\
\hline
\end{tabular}




\begin{tabular}{|c|c|}
\hline $\mathrm{C} 5-\mathrm{C} 6-\mathrm{C} 7-\mathrm{C} 9$ & $64.3(8)$ \\
\hline $\mathrm{C} 16-\mathrm{C} 11-\mathrm{C} 12-\mathrm{C} 13$ & $2.0(8)$ \\
\hline $\mathrm{C} 16-\mathrm{C} 11-\mathrm{C} 12-\mathrm{S} 2$ & $-171.2(4)$ \\
\hline $\mathrm{C} 12-\mathrm{C} 11-\mathrm{C} 16-\mathrm{C} 15$ & $5.1(8)$ \\
\hline $\mathrm{C} 12-\mathrm{C} 11-\mathrm{C} 16-\mathrm{C} 17$ & $-177.6(5)$ \\
\hline $\mathrm{S} 2-\mathrm{C} 12-\mathrm{C} 13-\mathrm{C} 14$ & $164.4(4)$ \\
\hline $\mathrm{C} 11-\mathrm{C} 12-\mathrm{C} 13-\mathrm{C} 14$ & $-8.8(7)$ \\
\hline $\mathrm{S} 2-\mathrm{C} 12-\mathrm{C} 13-\mathrm{O} 2$ & $-15.9(7)$ \\
\hline $\mathrm{C} 11-\mathrm{C} 12-\mathrm{C} 13-\mathrm{O} 2$ & $171.0(5)$ \\
\hline $\mathrm{O} 2-\mathrm{C} 13-\mathrm{C} 14-\mathrm{S} 3$ & $12.2(6)$ \\
\hline $\mathrm{O} 2-\mathrm{C} 13-\mathrm{C} 14-\mathrm{C} 15$ & $-171.0(4)$ \\
\hline $\mathrm{C} 12-\mathrm{C} 13-\mathrm{C} 14-\mathrm{C} 15$ & $8.7(6)$ \\
\hline $\mathrm{C} 12-\mathrm{C} 13-\mathrm{C} 14-\mathrm{S} 3$ & $-168.1(4)$ \\
\hline $\mathrm{C} 13-\mathrm{C} 14-\mathrm{C} 15-\mathrm{C} 16$ & $-1.9(7)$ \\
\hline $\mathrm{S} 3-\mathrm{C} 14-\mathrm{C} 15-\mathrm{C} 16$ & $174.9(4)$ \\
\hline $\mathrm{C} 14-\mathrm{C} 15-\mathrm{C} 16-\mathrm{C} 17$ & $177.5(5)$ \\
\hline $\mathrm{C} 14-\mathrm{C} 15-\mathrm{C} 16-\mathrm{C} 11$ & $-5.2(7)$ \\
\hline $\mathrm{C} 11-\mathrm{C} 16-\mathrm{C} 17-\mathrm{C} 18$ & $17.2(9)$ \\
\hline $\mathrm{C} 11-\mathrm{C} 16-\mathrm{C} 17-\mathrm{C} 19$ & $-104.6(7)$ \\
\hline $\mathrm{C} 15-\mathrm{C} 16-\mathrm{C} 17-\mathrm{C} 18$ & $-165.6(7)$ \\
\hline $\mathrm{C} 11-\mathrm{C} 16-\mathrm{C} 17-\mathrm{C} 20$ & $136.3(6)$ \\
\hline $\mathrm{C} 15-\mathrm{C} 16-\mathrm{C} 17-\mathrm{C} 19$ & $72.5(7)$ \\
\hline $\mathrm{C} 15-\mathrm{C} 16-\mathrm{C} 17-\mathrm{C} 20$ & $-46.6(8)$ \\
\hline $\mathrm{C} 26-\mathrm{C} 21-\mathrm{C} 22-\mathrm{S} 3$ & $-179.2(5)$ \\
\hline $\mathrm{C} 26-\mathrm{C} 21-\mathrm{C} 22-\mathrm{C} 23$ & $-2.0(9)$ \\
\hline $\mathrm{C} 22-\mathrm{C} 21-\mathrm{C} 26-\mathrm{C} 25$ & $2.5(9)$ \\
\hline $\mathrm{C} 22-\mathrm{C} 21-\mathrm{C} 26-\mathrm{C} 27$ & $-177.5(6)$ \\
\hline $\mathrm{C} 21-\mathrm{C} 22-\mathrm{C} 23-\mathrm{C} 24$ & $1.4(7)$ \\
\hline $\mathrm{S} 3-\mathrm{C} 22-\mathrm{C} 23-\mathrm{C} 24$ & $178.6(4)$ \\
\hline $\mathrm{C} 21-\mathrm{C} 22-\mathrm{C} 23-\mathrm{O} 3$ & $-178.5(5)$ \\
\hline $\mathrm{S} 3-\mathrm{C} 22-\mathrm{C} 23-\mathrm{O} 3$ & $-1.3(7)$ \\
\hline $\mathrm{C} 22-\mathrm{C} 23-\mathrm{C} 24-\mathrm{C} 25$ & $-1.6(7)$ \\
\hline $\mathrm{O} 3-\mathrm{C} 23-\mathrm{C} 24-\mathrm{C} 25$ & $178.3(5)$ \\
\hline $\mathrm{C} 22-\mathrm{C} 23-\mathrm{C} 24-\mathrm{S} 4$ & $174.5(4)$ \\
\hline $\mathrm{O} 3-\mathrm{C} 23-\mathrm{C} 24-\mathrm{S} 4$ & $-5.6(6)$ \\
\hline $\mathrm{C} 23-\mathrm{C} 24-\mathrm{C} 25-\mathrm{C} 26$ & $2.3(9)$ \\
\hline $\mathrm{S} 4-\mathrm{C} 24-\mathrm{C} 25-\mathrm{C} 26$ & $-173.8(5)$ \\
\hline $\mathrm{C} 24-\mathrm{C} 25-\mathrm{C} 26-\mathrm{C} 27$ & $177.3(6)$ \\
\hline $\mathrm{C} 24-\mathrm{C} 25-\mathrm{C} 26-\mathrm{C} 21$ & $-2.7(9)$ \\
\hline $\mathrm{C} 21-\mathrm{C} 26-\mathrm{C} 27-\mathrm{C} 30$ & $179.6(8)$ \\
\hline $\mathrm{C} 25-\mathrm{C} 26-\mathrm{C} 27-\mathrm{C} 29$ & $118.4(8)$ \\
\hline $\mathrm{C} 21-\mathrm{C} 26-\mathrm{C} 27-\mathrm{C} 29$ & $-61.6(8)$ \\
\hline $\mathrm{C} 21-\mathrm{C} 26-\mathrm{C} 27-\mathrm{C} 28$ & $58.9(8)$ \\
\hline $\mathrm{C} 25-\mathrm{C} 26-\mathrm{C} 27-\mathrm{C} 28$ & $-121.1(7)$ \\
\hline $\mathrm{C} 25-\mathrm{C} 26-\mathrm{C} 27-\mathrm{C} 30$ & $-0.4(10)$ \\
\hline $\mathrm{C} 32-\mathrm{C} 31-\mathrm{C} 36-\mathrm{C} 35$ & $-0.1(7)$ \\
\hline $\mathrm{C} 32-\mathrm{C} 31-\mathrm{C} 36-\mathrm{C} 37$ & $-179.2(4)$ \\
\hline $\mathrm{C} 36-\mathrm{C} 31-\mathrm{C} 32-\mathrm{C} 33$ & $-0.1(7)$ \\
\hline
\end{tabular}

\begin{tabular}{|c|c|}
\hline $\mathrm{O} 3^{\prime}-\mathrm{C} 23^{\prime}-\mathrm{C} 24^{\prime}-\mathrm{S} 4^{\prime}$ & $-5.6(6)$ \\
\hline $\mathrm{C} 22^{\prime}-\mathrm{C} 23^{\prime}-\mathrm{C} 24^{\prime}-\mathrm{C} 25^{\prime}$ & $-1.8(7)$ \\
\hline $\mathrm{O}^{\prime}-\mathrm{C} 23^{\prime}-\mathrm{C} 24^{\prime}-\mathrm{C} 25^{\prime}$ & $178.6(4)$ \\
\hline $\mathrm{C} 22^{\prime}-\mathrm{C} 23^{\prime}-\mathrm{C} 24^{\prime}-\mathrm{S} 4^{\prime}$ & $174.1(4)$ \\
\hline $\mathrm{C} 23^{\prime}-\mathrm{C} 24^{\prime}-\mathrm{C} 25^{\prime}-\mathrm{C} 26^{\prime}$ & $1.8(8)$ \\
\hline $\mathrm{S} 4^{\prime}-\mathrm{C} 24^{\prime}-\mathrm{C} 25^{\prime}-\mathrm{C} 26^{\prime}$ & $-174.0(4)$ \\
\hline $\mathrm{C} 24^{\prime}-\mathrm{C} 25^{\prime}-\mathrm{C} 26^{\prime}-\mathrm{C} 27^{\prime}$ & $177.2(5)$ \\
\hline $\mathrm{C} 24^{\prime}-\mathrm{C} 25^{\prime}-\mathrm{C} 26^{\prime}-\mathrm{C} 21^{\prime}$ & $-1.7(8)$ \\
\hline $\mathrm{C} 21^{\prime}-\mathrm{C} 26^{\prime}-\mathrm{C} 27^{\prime}-\mathrm{C} 29^{\prime}$ & $-61.8(8)$ \\
\hline $\mathrm{C} 25^{\prime}-\mathrm{C} 26^{\prime}-\mathrm{C} 27^{\prime}-\mathrm{C} 30^{\prime}$ & $-1.7(8)$ \\
\hline $\mathrm{C} 25^{\prime}-\mathrm{C} 26^{\prime}-\mathrm{C} 27^{\prime}-\mathrm{C} 28^{\prime}$ & $-120.6(6)$ \\
\hline $\mathrm{C} 21^{\prime}-\mathrm{C} 26^{\prime}-\mathrm{C} 27^{\prime}-\mathrm{C} 28^{\prime}$ & $58.3(8)$ \\
\hline $\mathrm{C} 25^{\prime}-\mathrm{C} 26^{\prime}-\mathrm{C} 27^{\prime}-\mathrm{C} 29^{\prime}$ & $119.3(6)$ \\
\hline $\mathrm{C} 21^{\prime}-\mathrm{C} 26^{\prime}-\mathrm{C} 27^{\prime}-\mathrm{C} 30^{\prime}$ & $177.2(6)$ \\
\hline $\mathrm{C} 36^{\prime}-\mathrm{C} 31^{\prime}-\mathrm{C} 32^{\prime}-\mathrm{S} 4^{\prime}$ & $174.1(4)$ \\
\hline $\mathrm{C} 32^{\prime}-\mathrm{C} 31^{\prime}-\mathrm{C} 36^{\prime}-\mathrm{C} 37^{\prime}$ & $-177.8(5)$ \\
\hline $\mathrm{C} 36^{\prime}-\mathrm{C} 31^{\prime}-\mathrm{C} 32^{\prime}-\mathrm{C} 33^{\prime}$ & $-0.2(7)$ \\
\hline $\mathrm{C} 32^{\prime}-\mathrm{C} 31^{\prime}-\mathrm{C} 36^{\prime}-\mathrm{C} 35^{\prime}$ & $-0.2(7)$ \\
\hline $\mathrm{S} 4^{\prime}-\mathrm{C} 32^{\prime}-\mathrm{C} 33^{\prime}-\mathrm{C} 34^{\prime}$ & $-173.0(4)$ \\
\hline $\mathrm{S} 44^{\prime}-\mathrm{C} 32^{\prime}-\mathrm{C} 33^{\prime}-\mathrm{O} 4^{\prime}$ & $7.6(6)$ \\
\hline $\mathrm{C} 31^{\prime}-\mathrm{C} 32^{\prime}-\mathrm{C} 33^{\prime}-\mathrm{O} 4^{\prime}$ & $-178.2(4)$ \\
\hline $\mathrm{C} 31^{\prime}-\mathrm{C} 32^{\prime}-\mathrm{C} 33^{\prime}-\mathrm{C} 34^{\prime}$ & $1.3(7)$ \\
\hline $\mathrm{O} 4^{\prime}-\mathrm{C} 33^{\prime}-\mathrm{C} 34^{\prime}-\mathrm{S} 1^{\prime}$ & $-8.5(6)$ \\
\hline $\mathrm{C} 32^{\prime}-\mathrm{C} 33^{\prime}-\mathrm{C} 34^{\prime}-\mathrm{C} 35^{\prime}$ & $-1.9(7)$ \\
\hline $\mathrm{C} 32^{\prime}-\mathrm{C} 33^{\prime}-\mathrm{C} 34^{\prime}-\mathrm{S} 1^{\prime}$ & $172.1(4)$ \\
\hline $\mathrm{O} 4^{\prime}-\mathrm{C} 33^{\prime}-\mathrm{C} 34^{\prime}-\mathrm{C} 35^{\prime}$ & $177.6(5)$ \\
\hline $\mathrm{C} 33^{\prime}-\mathrm{C} 34^{\prime}-\mathrm{C} 35^{\prime}-\mathrm{C} 36^{\prime}$ & $1.4(8)$ \\
\hline $\mathrm{S} 1^{\prime}-\mathrm{C} 34^{\prime}-\mathrm{C} 35^{\prime}-\mathrm{C} 36^{\prime}$ & $-172.4(4)$ \\
\hline $\mathrm{C} 34^{\prime}-\mathrm{C} 35^{\prime}-\mathrm{C} 36^{\prime}-\mathrm{C} 31^{\prime}$ & $-0.4(8)$ \\
\hline $\mathrm{C} 34^{\prime}-\mathrm{C} 35^{\prime}-\mathrm{C} 36^{\prime}-\mathrm{C} 37^{\prime}$ & $177.2(5)$ \\
\hline $\mathrm{C} 31^{\prime}-\mathrm{C} 36^{\prime}-\mathrm{C} 37^{\prime}-\mathrm{C} 39^{\prime}$ & $-104.3(8)$ \\
\hline $\mathrm{C} 31^{\prime}-\mathrm{C} 36^{\prime}-\mathrm{C} 37^{\prime}-\mathrm{C} 40^{\prime}$ & $134.9(6)$ \\
\hline $\mathrm{C} 31^{\prime}-\mathrm{C} 36^{\prime}-\mathrm{C} 37^{\prime}-\mathrm{C} 38^{\prime}$ & $16.0(9)$ \\
\hline $\mathrm{C} 35^{\prime}-\mathrm{C} 36^{\prime}-\mathrm{C} 37^{\prime}-\mathrm{C} 39^{\prime}$ & $78.2(9)$ \\
\hline $\mathrm{C} 35^{\prime}-\mathrm{C} 36^{\prime}-\mathrm{C} 37^{\prime}-\mathrm{C} 40^{\prime}$ & $-42.6(8)$ \\
\hline $\mathrm{C} 35^{\prime}-\mathrm{C} 36^{\prime}-\mathrm{C} 37^{\prime}-\mathrm{C} 38^{\prime}$ & $-161.6(7)$ \\
\hline $\mathrm{O} 4^{\prime}-\mathrm{C} 41^{\prime}-\mathrm{C} 42^{\prime}-\mathrm{O}^{\prime}$ & $0.4(8)$ \\
\hline $\mathrm{O} 4^{\prime}-\mathrm{C} 41^{\prime}-\mathrm{C} 42^{\prime}-\mathrm{C} 43^{\prime}$ & $-177.3(5)$ \\
\hline $\mathrm{O} 5^{\prime}-\mathrm{C} 42^{\prime}-\mathrm{C} 43^{\prime}-\mathrm{C} 44^{\prime}$ & $-0.4(9)$ \\
\hline $\mathrm{O}^{\prime}-\mathrm{C} 42^{\prime}-\mathrm{C} 43^{\prime}-\mathrm{C} 48^{\prime}$ & $-179.7(7)$ \\
\hline $\mathrm{C} 41^{\prime}-\mathrm{C} 42^{\prime}-\mathrm{C} 43^{\prime}-\mathrm{C} 44^{\prime}$ & $177.3(5)$ \\
\hline $\mathrm{C} 41^{\prime}-\mathrm{C} 42^{\prime}-\mathrm{C} 43^{\prime}-\mathrm{C} 48^{\prime}$ & $-2.0(9)$ \\
\hline $\mathrm{C} 42^{\prime}-\mathrm{C} 43^{\prime}-\mathrm{C} 44^{\prime}-\mathrm{C} 45^{\prime}$ & $-179.8(6)$ \\
\hline $\mathrm{C} 48^{\prime}-\mathrm{C} 43^{\prime}-\mathrm{C} 44^{\prime}-\mathrm{C} 45^{\prime}$ & $-0.6(9)$ \\
\hline $\mathrm{C} 42^{\prime}-\mathrm{C} 43^{\prime}-\mathrm{C} 48^{\prime}-\mathrm{C} 47^{\prime}$ & $-179.7(6)$ \\
\hline $\mathrm{C} 44^{\prime}-\mathrm{C} 43^{\prime}-\mathrm{C} 48^{\prime}-\mathrm{C} 47^{\prime}$ & $1.1(9)$ \\
\hline $\mathrm{C} 43^{\prime}-\mathrm{C} 44^{\prime}-\mathrm{C} 45^{\prime}-\mathrm{C} 46^{\prime}$ & $0.6(10)$ \\
\hline $\mathrm{C} 44^{\prime}-\mathrm{C} 45^{\prime}-\mathrm{C} 46^{\prime}-\mathrm{C} 47^{\prime}$ & $-1.1(11)$ \\
\hline
\end{tabular}




$\begin{array}{ll}\mathrm{C} 36-\mathrm{C} 31-\mathrm{C} 32-\mathrm{S} 4 & 174.0(3) \\ \mathrm{C} 31-\mathrm{C} 32-\mathrm{C} 33-\mathrm{C} 34 & 1.1(7) \\ \mathrm{C} 31-\mathrm{C} 32-\mathrm{C} 33-\mathrm{O} 4 & -178.8(4) \\ \mathrm{S} 4-\mathrm{C} 32-\mathrm{C} 33-\mathrm{O} 4 & 7.2(6) \\ \mathrm{S} 4-\mathrm{C} 32-\mathrm{C} 33-\mathrm{C} 34 & -173.0(4) \\ \mathrm{C} 32-\mathrm{C} 33-\mathrm{C} 34-\mathrm{C} 35 & -1.9(7) \\ \mathrm{O} 4-\mathrm{C} 33-\mathrm{C} 34-\mathrm{S} 1 & -8.0(6) \\ \mathrm{C} 32-\mathrm{C} 33-\mathrm{C} 34-\mathrm{S} 1 & 172.2(4) \\ \mathrm{O} 4-\mathrm{C} 33-\mathrm{C} 34-\mathrm{C} 35 & 178.0(5) \\ \mathrm{C} 33-\mathrm{C} 34-\mathrm{C} 35-\mathrm{C} 36 & 1.7(8) \\ \mathrm{S} 1-\mathrm{C} 34-\mathrm{C} 35-\mathrm{C} 36 & -172.4(4) \\ \mathrm{C} 34-\mathrm{C} 35-\mathrm{C} 36-\mathrm{C} 31 & -0.7(7) \\ \mathrm{C} 34-\mathrm{C} 35-\mathrm{C} 36-\mathrm{C} 37 & 178.4(5) \\ \mathrm{C} 35-\mathrm{C} 36-\mathrm{C} 37-\mathrm{C} 40 & -41.7(7) \\ \mathrm{C} 31-\mathrm{C} 36-\mathrm{C} 37-\mathrm{C} 40 & 137.3(6) \\ \mathrm{C} 31-\mathrm{C} 36-\mathrm{C} 37-\mathrm{C} 38 & 17.0(8) \\ \mathrm{C} 31-\mathrm{C} 36-\mathrm{C} 37-\mathrm{C} 39 & -102.9(7) \\ \mathrm{C} 35-\mathrm{C} 36-\mathrm{C} 37-\mathrm{C} 38 & -162.1(6) \\ \mathrm{C} 35-\mathrm{C} 36-\mathrm{C} 37-\mathrm{C} 39 & 78.0(7) \\ \mathrm{O} 4-\mathrm{C} 41-\mathrm{C} 42-\mathrm{O} 5 & 3.1(8) \\ \mathrm{O} 4-\mathrm{C} 41-\mathrm{C} 42-\mathrm{C} 43 & -176.5(5) \\ \mathrm{C} 41-\mathrm{C} 42-\mathrm{C} 43-\mathrm{C} 44 & 177.2(5) \\ \mathrm{C} 41-\mathrm{C} 42-\mathrm{C} 43-\mathrm{C} 48 & -3.0(9) \\ \mathrm{O} 5-\mathrm{C} 42-\mathrm{C} 43-\mathrm{C} 48 & 177.5(7) \\ \mathrm{O} 5-\mathrm{C} 42-\mathrm{C} 43-\mathrm{C} 44 & -2.3(10) \\ \mathrm{C} 44-\mathrm{C} 43-\mathrm{C} 48-\mathrm{C} 47 & -0.3(9) \\ & \end{array}$

$\begin{array}{ll}\mathrm{C} 45^{\prime}-\mathrm{C} 46^{\prime}-\mathrm{C} 47^{\prime}-\mathrm{C} 48^{\prime} & 1.6(11) \\ \text { C46'-C47'-C48'-C43' } & -1.6(9) \\ \text { C103-N1-C101-C102 } & -59.0(13) \\ \text { C105-N1-C101-C102 } & 171.7(12) \\ \text { C107-N1-C101-C102 } & 59.9(13) \\ \text { C101-N1-C103-C104 } & -51.6(13) \\ \text { C105-N1-C103-C104 } & 70.0(14) \\ \text { C107-N1-C103-C104 } & -170.0(11) \\ \text { C101-N1-C105-C106 } & 179.2(13) \\ \text { C103-N1-C105-C106 } & 55.6(17) \\ \text { C107-N1-C105-C106 } & -66.6(16) \\ \text { C101-N1-C107-C108 } & 71.3(15) \\ \text { C103-N1-C107-C108 } & -169.4(14) \\ \text { C105-N1-C107-C108 } & -41.0(17) \\ \text { C203-N2-C201-C202 } & -60.6(12) \\ \text { C205-N2-C201-C202 } & 178.7(10) \\ \text { C207-N2-C201-C202 } & 59.9(12) \\ \text { C201-N2-C203-C204 } & -53.0(11) \\ \text { C205-N2-C203-C204 } & 63.3(11) \\ \text { C207-N2-C203-C204 } & -173.9(10) \\ \text { C201-N2-C205-C206 } & -175.4(9) \\ \text { C203-N2-C205-C206 } & 64.0(11) \\ \text { C207-N2-C205-C206 } & -57.6(12) \\ \text { C201-N2-C207-C208 } & 67.7(14) \\ \text { C203-N2-C207-C208 } & -170.1(12) \\ \text { C205-N2-C207-C208 } & -46.8(15)\end{array}$

Hydrogen-bond geometry $\left(A,{ }^{\circ}\right)$

\begin{tabular}{|c|c|c|c|c|}
\hline$D-\mathrm{H} \cdots A$ & $D-\mathrm{H}$ & $\mathrm{H} \cdots A$ & $D \cdots A$ & $D-\mathrm{H} \cdots A$ \\
\hline $\mathrm{O} 1-\mathrm{H} 1 O \cdots \mathrm{S} 2$ & 0.82 & 2.61 & $3.110(4)$ & 121 \\
\hline $\mathrm{O} 1-\mathrm{H} 1 O \cdots \mathrm{O} 2$ & 0.82 & 1.85 & $2.581(6)$ & 147 \\
\hline $\mathrm{O} 2-\mathrm{H} 2 O \cdots \mathrm{Cl} 2$ & 0.82 & 2.30 & $3.048(10)$ & 152 \\
\hline $\mathrm{O} 2-\mathrm{H} 2 O \cdots \mathrm{S} 2$ & 0.82 & 2.70 & $3.051(4)$ & 108 \\
\hline $\mathrm{O} 3-\mathrm{H} 3 O \cdots \mathrm{S} 3$ & 0.82 & 2.57 & $3.077(4)$ & 121 \\
\hline $\mathrm{O} 3-\mathrm{H} 3 \mathrm{O} \cdots \mathrm{O} 2$ & 0.82 & 1.91 & $2.567(6)$ & 136 \\
\hline $\mathrm{O} 1^{\prime}-\mathrm{H} 6 O^{\cdots} \cdots \mathrm{S} 2^{\prime}$ & 0.82 & 2.60 & $3.105(4)$ & 121 \\
\hline $\mathrm{O} 1^{\prime}-\mathrm{H} 6 O \cdots \mathrm{O} 2^{\prime}$ & 0.82 & 1.85 & $2.580(5)$ & 147 \\
\hline $\mathrm{O} 1^{\prime}-\mathrm{H} 6{ }^{\cdots}{ }^{\cdots} \mathrm{C} 11^{\mathrm{i}}$ & 0.82 & 2.81 & $3.338(11)$ & 124 \\
\hline $\mathrm{O} 2^{\prime}-\mathrm{H} 7 O^{\cdots} \cdot \mathrm{S} 2^{\prime}$ & 0.82 & 2.68 & $3.044(4)$ & 109 \\
\hline $\mathrm{O} 2^{\prime}-\mathrm{H} 7 O^{\cdots} \mathrm{Cl}^{\mathrm{i}}$ & 0.82 & 2.27 & $3.006(10)$ & 150 \\
\hline $\mathrm{O}^{\prime}-\mathrm{H} 8 O^{\cdots} \cdot \mathrm{S} 3^{\prime}$ & 0.82 & 2.57 & $3.078(4)$ & 121 \\
\hline $\mathrm{O} 3^{\prime}-\mathrm{H} 8 \mathrm{O}^{\cdots} \cdot \mathrm{O} 2^{\prime}$ & 0.82 & 1.90 & $2.567(5)$ & 138 \\
\hline $\mathrm{C} 103-\mathrm{H} 10 L \cdots \mathrm{Cl} 2$ & 0.97 & 2.43 & $3.397(17)$ & 174 \\
\hline $\mathrm{C} 106-\mathrm{H} 10 V \cdots \mathrm{Cl} 2$ & 0.96 & 2.64 & $3.382(19)$ & 135 \\
\hline $\mathrm{C} 202-\mathrm{H} 20 I \cdots \mathrm{O} 5$ & 0.96 & 2.58 & $3.459(16)$ & 152 \\
\hline $\mathrm{C} 203-\mathrm{H} 20 L \cdots \mathrm{C} 11^{\mathrm{ii}}$ & 0.97 & 2.41 & $3.373(15)$ & 173 \\
\hline $\mathrm{C} 204-\mathrm{H} 20 P \cdots \mathrm{Cl} 2^{\mathrm{ii}}$ & 0.96 & 2.78 & $3.63(2)$ & 147 \\
\hline
\end{tabular}




\begin{tabular}{|c|c|c|c|c|}
\hline $\mathrm{C} 206-\mathrm{H} 20 V \cdots \mathrm{Cl} 1^{\mathrm{ii}}$ & 0.96 & 2.55 & $3.353(18)$ & 141 \\
\hline $\mathrm{C} 41-\mathrm{H} 41 A \cdots \mathrm{O} 3$ & 0.97 & 2.45 & $3.318(6)$ & 149 \\
\hline $\mathrm{C} 41-\mathrm{H} 41 B \cdots \mathrm{O} 1$ & 0.97 & 2.46 & $3.253(6)$ & 139 \\
\hline $\mathrm{C} 41^{\prime}-\mathrm{H} 41 C^{\prime} \cdots \mathrm{S} 4^{\prime}$ & 0.97 & 2.87 & $3.414(6)$ & 116 \\
\hline $\mathrm{C} 41^{\prime}-\mathrm{H} 41 C^{\cdots} \cdot \mathrm{O}^{\prime}$ & 0.97 & 2.45 & $3.313(6)$ & 148 \\
\hline $\mathrm{C} 41^{\prime}-\mathrm{H} 41 D^{\cdots} \mathrm{O}^{\prime}$ & 0.97 & 2.47 & $3.265(6)$ & 139 \\
\hline $\mathrm{C} 44-\mathrm{H} 44 \cdots \mathrm{O} 2^{\mathrm{iii}}$ & 0.93 & 2.54 & $3.431(7)$ & 160 \\
\hline $\mathrm{C} 44^{\prime}-\mathrm{H} 44^{\prime} \cdots \mathrm{O} 2^{\prime \text {,iv }}$ & 0.93 & 2.57 & $3.447(6)$ & 158 \\
\hline $\mathrm{C} 47-\mathrm{H} 47 \cdots \mathrm{O}^{\mathrm{v}}$ & 0.93 & 2.55 & $3.368(6)$ & 146 \\
\hline $\mathrm{C} 47-\mathrm{H} 47 \cdots \mathrm{O}^{\mathrm{v}}$ & 0.93 & 2.45 & $3.255(7)$ & 145 \\
\hline $\mathrm{C} 47^{\prime}-\mathrm{H} 47^{\prime} \cdots \mathrm{O} 4^{\prime v i}$ & 0.93 & 2.57 & $3.380(6)$ & 146 \\
\hline $\mathrm{C} 47^{\prime}-\mathrm{H} 47^{\prime} \cdots \mathrm{O} 5^{\prime \mathrm{vi}}$ & 0.93 & 2.47 & $3.277(7)$ & 145 \\
\hline
\end{tabular}

Symmetry codes: (i) $-x+1, y+1 / 2,-z+1$; (ii) $x, y+1, z$; (iii) $-x+2, y+1 / 2,-z+2$; (iv) $-x+1, y-1 / 2,-z$; (v) $-x+2, y-1 / 2,-z+2$; (vi) $-x+1, y+1 / 2,-z$. 